\title{
NOTESKB: WEB-BASED SEMANTIC ANNOTATION TOOL FOR ONLINE MULTIMEDIA LEARNING CONTENT
}

\author{
by \\ Abdulrahman Alamoudi
}

\author{
A thesis submitted to \\ the Faculty of Graduate Studies and Postdoctoral \\ Affairs in partial fulfillment of \\ the requirements for the degree of \\ MASTER OF COMPUTER SCIENCE \\ Human-Computer Interaction \\ at \\ CARLETON UNIVERSITY \\ Ottawa, Ontario \\ September, 2017
}

(c) Copyright by Abdulrahman Alamoudi, 2017 


\section{Contents}

List of Tables $\quad$ v

List of Figures $\quad$ vi

Abstract viii

Acknowledgements $\quad$ ix

Chapter 1 Introduction 1

1.1 Research Goal . . . . . . . . . . . . . . . . . . . 4

1.2 Research Questions . . . . . . . . . . . . . . . 5

1.3 Proposed Solution . . . . . . . . . . . . . . . . . . 6

1.4 Specific Objectives . . . . . . . . . . . . . . 6

1.5 Outcomes ........................ 6

1.6 Thesis Structure . . . . . . . . . . . . . . . . . . . 7

1.7 Motivation . . . . . . . . . . . . . . . . 8

$\begin{array}{lll}\text { Chapter } 2 & \text { Background } & 9\end{array}$

2.1 Knowledge \& Learning . . . . . . . . . . . . . . . . . . . . . . . . 11

2.1.1 Learning Theories . . . . . . . . . . . . . . . . . . . 12

2.1.2 Related Concepts . . . . . . . . . . . . . . . 15

2.2 Semantic Web . . . . . . . . . . . . . . . . . 18

2.2.1 Ontologies . . . . . . . . . . . . . . 20

2.2.2 Linked Open Data (LOD) . . . . . . . . . . . . . 22

2.3 Semantic Annotation . . . . . . . . . . . . . . . . 23

Chapter $3 \quad$ Design and Implementation 28

3.1 Proposed Design Basis . . . . . . . . . . . . . . . . . 28

3.1.1 UI Components . . . . . . . . . . . . . . . . . . . . 28 
3.1.2 Note-taking Style (Annotation Style) . . . . . . . . . . . . . 30

3.1 .3 Wikification Process . . . . . . . . . . . . . . 30

3.2 User Interface Design . . . . . . . . . . . . . . . . . . . . 31

3.2.1 UI Components . . . . . . . . . . . . . . . 33

3.2 .2 Features . . . . . . . . . . . . . . . . . . 38

3.2.3 Related Concepts and Techniques . . . . . . . . . . . . . . 39

3.3 System Architecture and Implementation . . . . . . . . . . . . . . . . 44

3.3.1 Back-end architecture . . . . . . . . . . . . . . 44

3.3.2 Front-end architecture . . . . . . . . . . . . . . 46

$\begin{array}{lll}\text { Chapter } 4 & \text { Methodology } & 49\end{array}$

4.1 Participants . . . . . . . . . . . . . . . . . 50

4.2 Materials . . . . . . . . . . . . . . . . . . 51

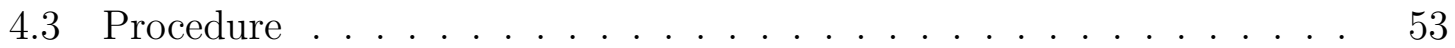

4.3.1 Part 1: Pre-test Questionnaires ............ 53

4.3.2 Part 2: Note-taking Sessions . . . . . . . . . . . 54

4.3.3 Part 3: UI Actions . . . . . . . . . . . . . . 57

4.3.4 Part 4: Interview . . . . . . . . . . . . . . . . 59

4.4 Analysis Plan . . . . . . . . . . . . . . . . . . . . 59

4.4.1 Independent Variables (Factors) . . . . . . . . . . . . . 60

4.4.2 Dependent Variables (Measures) . . . . . . . . . . . . 61

$\begin{array}{lll}\text { Chapter } 5 & \text { Results and Discussion } & 63\end{array}$

5.1 Results......................... 63

5.1.1 Descriptive Statistics . . . . . . . . . . . . . . . 64

5.1 .2 Comparisons . . . . . . . . . . . . . . . . . . 72

5.1.3 Qualitative Results . . . . . . . . . . . . . . . . . 74

5.2 Interpretation and Discussion _. . . . . . . . . . . 77

5.2.1 Annotation Method Support . . . . . . . . . . . 77

5.2 .2 Annotation Style . . . . . . . . . . . . . . . . 78

5.2.3 Learning Content . . . . . . . . . . . . . . . . 79 
5.2.4 User Competence . . . . . . . . . . . . . . . . . . . 80

5.2.5 Reflections .................... 81

$\begin{array}{lll}\text { Chapter } 6 & \text { Conclusion } & 82\end{array}$

6.1 Research Outcomes . . . . . . . . . . . . . . . 83

6.1.1 Main Findings . . . . . . . . . . . . . . . . 83

6.1.2 Limitations . . . . . . . . . . . . . . . . . 84

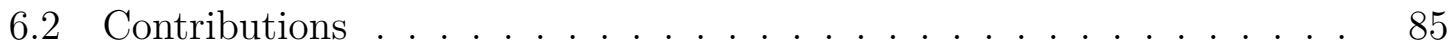

6.3 Future Work . . . . . . . . . . . . . . . . . . 85

$\begin{array}{lll}\text { Appendix A } & \text { Annotation-Types } & 86\end{array}$

$\begin{array}{lll}\text { Appendix B } & \text { Pre-Test Questionnaires } & 87\end{array}$

Appendix C Post-Test Questionnaires $\quad 95$

$\begin{array}{lll}\text { Appendix D Video Transcripts } & 107\end{array}$

D.1 Video-A Transcript . . . . . . . . . . . . . . . . 107

D.2 Video-B Transcript . . . . . . . . . . . . . . . . . . . 109

D.3 Video-C Transcript . . . . . . . . . . . . . . . . . . . 111

$\begin{array}{lll}\text { Appendix E Examination Supplements } & 114\end{array}$

$\begin{array}{lll}\text { Appendix F } & \text { Interview Sample } & 116\end{array}$

$\begin{array}{ll}\text { Bibliography } & 127\end{array}$ 


\section{List of Tables}

2.1 Summary of the reviewed annotation tools. . . . . . . . . . 26

4.1 Table of the independent variables along with their levels. . . . 61

4.2 Table with the study trials ordered as they were experienced by both participant groups. . . . . . . . . . . . . . 61

5.1 Summaries of the answers during the interviews with participants. 64 SUS results. . . . . . . . . . . . . . . . 70 ASQ results. .................... . . 71

5.4 Results from the Wilcoxon rank test. . . . . . . . . . . . . 73

5.5 Summary of SUS scores. . . . . . . . . . . . . 78

$5.6 \quad$ Summary of ASQ scores. . . . . . . . . . . 78

A.1 Descriptions of the underlying meaning of annotation-types. . . 86 


\section{List of Figures}

1.1 Overview of the different aspects of the research. . . . . . . 5

2.1 Categories of domain knowledge. . . . . . . . . . . . 12

$2.2 \quad$ Learning by Doing Cycle. . . . . . . . . . . . . 16

2.3 Categories of Annotations by Dan Whaley [101] . . . . . . . . 24

2.4 Model of Multimedia Annotation Tools [101] . . . . . . . . 25

3.1 Preview of NotesKB's early designs for the panel UI element in the Google Chrome browser. . . . . . . . . . . . . . . 32

3.2 An overview of the Library View view. . . . . . . . . . . . . . 34

3.3 Initializing NotesKB via Google Chrome browser's extension. . 35

$3.4 \quad$ An overview of the Notepad View view. . . . . . . . . . . . . 36

3.5 Steps involved in linking the hashtag \#Nile inside of the Hashtag Linking View as part of the Wikification process. . . . . . . . 37

3.6 Simplified architecture of NotesKB video annotation system. . 44

3.7 The class diagram of NotesKB's back-end. . . . . . . . . . 45

3.8 UI interaction flowchart diagram. . . . . . . . . . . . . 47

4.1 Overview of the testing setting - which includes (A) the main monitor display, (B) an eye-tracker, (C) a microphone, and (D) an input space [for mouse and keyboard also for PnP]. . . . . 51

4.2 Overview of procedure taken place during each testing session. $\quad 55$

$5.1 \quad$ Graphical representations of demographic data. . . . . . . 65

5.2 Graphical representations of data of the students' academic backgrounds and general skills. . . . . . . . . . . 65

5.3 Participants' ratings of their computer skills. . . . . . . . 66

$5.4 \quad$ Usage patterns of different activities on the Web. . . . . . 67

5.5 Participants' ratings of their note-taking skills and habits. . . 67

5.6 Boxplots displaying the distribution of collected SUS scores. . 68 
5.8 Barplots show the completion rate by participants carrying out the Wikification process as part of Action 4. . . . . . . . . 


\begin{abstract}
Due to the rapid growth of e-Learning contents, especially videos, published online, the usability of tools that help students keep track of such contents along with their thought traces is an issue. We addressed the problem by designing and building a web-based semantic annotation tool called NotesKB. Through technical and theoretical lenses, we aim to discover and improve upon the usability of such tools. The usability of NotesKB is evaluated and compared with two other predominant note-taking methods, namely Pen and Paper (PnP) and Microsoft OneNote. According to the SUS test scores, PnP is the most usable while NotesKB was the most preferred when students were interviewed. We have reaffirmed that cognitive load is key to design e-Learning systems and that note-taking tools with semantic capabilities need to help users learn how to use the greater power available.
\end{abstract}




\section{Acknowledgements}

First and foremost, I am grateful to my supervisors Dwight Deugo and Robert Biddle. It is due to their excellent guidance and support during this process and most of all for allowing me to pursue such project. Dr. Deugo, you had let me into the program and gave me the chance to embark on this remarkable journey to learn and develop. Dr. Biddle, I cannot thank you enough for accepting to co-supervise me half-way of my program and devoting an enormous amount of your valuable time - including weekends and holidays - just so that I finish writing my thesis. You have provided me with a first-hand experience of what a rigorous research is. I am also thankful to the members of my committee, Sonia Chiasson, Ali Arya, and the defense chair Anil Somayaji for their guidance, for their expertise, and for offering different perspectives, all of which have helped me shape this dissertation.

I would like to give special thanks to Ms. Erenia Oliver for all the help and assistance she had provided me when I needed it the most; I would always remember your kindness. Also, my thanks go to my colleagues in the HotSoft lab who helped with the studies, listened to presentations, and offered valuable feedback and insight throughout the process.

To my dear family back home, despite the thousands of miles that set us apart, you have always been in mind and heart and thank you for all the love you gave me, I shall carry it with me as long as I live. To Mr. \& Mrs. Brown, living in your happy household for the past nine years had been my privilege; and for that, I am indebted to you. Also, to my beloved cousins Elaf \& Mowaffaq, I will always cherish the moments we shared together, those memories are engraved in me, thank you for all your kindness. To my friend Faris, despite your busy schedule and responsibilities, you have always managed to stay in touch and ask about my wellbeing; thank you.

I am very thankful to the Saudi Arabian Cultural Bureau in Canada, and by extension the Saudi Arabian Government; for their ongoing help throughout my university years. 


\section{Chapter 1}

\section{Introduction}

The Internet provides an abundance of information to explore and select produced by various sources. It is estimated that roughly 2.5 quintillion bytes of data are produced every day; however, about $80 \%$ of produced data is unstructured and unorganized [41]. A tremendous number and variety of resources suitable for learning exist on the web nowadays; they open the door for learners to investigate any topic they wish to study in a wider, deeper, and timely way [43]. Those resources come in multiple formats (text, audio or video), but video resources stand out as being quite powerful and effective in learning environments due to their engaging nature [84]. Although the usage of videos has shown to be useful in that process, it is deemed insufficient on its own without the student being engaged with the learning material and cognitively active during the learning process [25]. According to Schwartz and Hartman [84], engagement refers to the ability to create "the mental context that prepares people to learn;" and there are two approaches to increase one of a learner: a) developing the student's interest in the learning material, and b) contextualizing it. In the former, engagement is approached by letting the learners get motivated about the learning content, thus develop their interest in the subject they are studying. In the other approach, providing a context to the learning subject that would support the students to find the subject to be of relevance and meaning. For instance, teachers would give the students some examples or some background information that is relevant to the students' daily lives.

The mentioned rapid growth in educational video content published online, it gave way for video annotation software to help students create and share their reflections about the content [62]. In the literature, there are hints of an existing relationship between note-taking (i.e., annotating) and active learning engagement [62, 79, 45]. To 
the development side of the online note-taking activity, the technology that allows utilizing it for learning is already there and is progressing [62]. As part of a study by Bargeron et al. [7], a prototype system called MRAS (Microsoft Research Annotation System) was developed as a Web-based video annotation tool; then it was compared to the traditional way taking note using pen and paper (referred to here as $\mathrm{PnP}$ ). The researchers found that in personal note-taking users preferred the software tool more than PnP. Thus, using annotation as a mean to achieve learning is thought to have potential.

According to Andrews et al. [5], the storage of an annotation element can take one of four forms: tag, attribute, relation or ontology. Using the ontology model, when annotating, is considered to be the most sophisticated and at the same time the most complex; this is usually referred to as semantic annotation. A technical definition of semantic annotation by Kiryakov et al. [49], describes it as "a specific metadata generation and usage schema, aiming to enable new information access methods and to extend the existing ones." Two main challenges face annotators when using this model, namely usability and maintenance of the ontology's conceptual model. In this thesis project, we intend to deal with the former. On the other hand, the other (nonsemantic) annotation models are usually observed in most traditional text annotation tools that use techniques such as free-text keywords or predefined vocabulary tagging; these have limited reliability and efficiency when dealing with the problem of semantic heterogeneity and data interoperability [5, 35]. Issues such as limited accuracy, disambiguation, completeness and lack of localization (i.e., multilinguality) support are common in systems that use non-semantic tagging $[64,35]$. The following list includes four of their main issues [5]:

- Base form variation: identified by using one word in different forms (e.g., plural vs. singular) or erroneously (e.g., misspellings). This issue is usually handled by applying a lemmatization procedure to the annotation's text.

- Polysemy: identified by having more than one possible interpretation of some text. For example, "orange" could refer to the color as well as the fruit. Another example is the use of the word "Java" which can relate to either the programming 
language or the Indonesian island.

- Synonymy: identified by having two concepts that differ syntactically but share the same connotation. For example, in a certain context, the word "image" is synonymous with the word "picture."

- Specificity gap: identified by having two concepts that one is included in the other as a subclass. For example, the word "cheddar," which is a kind of cheese, should appear in the search results when someone searches for the word "cheese."

Expressing semantics is not a new field of research to computer science, it can be dated back to the 1960's [33]. With the introduction of the Semantic Web (SW) [10], however, the landscape changed drastically. It is a continuing effort, and it is regarded sometimes as the second wave of the original World Wide Web (WWW or Web). In the early days of the WWW, interlinking documents relied on text, unlike in the SW which uses semantics (i.e. "ontologies") as a basis. Relying on ontologies to produce data would primarily result in populating the Web with information that is machine-readable $[10,103,33]$. Over the years, a set of principles and practices concerned with creating ontologies and content sharing from various domains has emerged, most notably Linked Data $[12,73]$. Applications implemented with the Linked Data principles in mind offer large potential in improving search efficiency and making the problem of data interoperability less severe, and eases the interpretation of a given concept shared with different parties [19, 26, 35, 87]. A number of web services, such as DBpedia and Freebase, provide semantic data as part of a large interconnected knowledge base known as the Linked Open Data (LOD).

We also have been looking at the user interfaces (UIs) of some academic and nonacademic web-based video annotation projects and investigated approaches they used to build annotations with LODs being infused into them. In the literature review and analysis done by Khan et al. [46], many of the reviewed video annotation tools are limited in their abilities to exploit the full potential of collected annotations, as we will discuss later in Chapter 2. Their lack of a search functionality or the ability to establish relationships between annotations are just examples of those limitations. They concluded their analysis by suggesting some requirements that we need to 
consider when developing UIs for video-annotation web applications, such as:

- sufficient incorporation of semantics to facilitate converting annotations' text into semantic annotations;

- searching functionality that allows the user to look for a specific thing within a video; and

- summarizing functionality according to an object or event.

\section{$1.1 \quad$ Research Goal}

The general goal of our research is to understand the activity of note-taking in general, and taking notes of online educational videos in particular. Choosing video as a medium from other educational multimedia material is due to the variety of them delivering educational content online as well as their effectiveness at doing so. Achieving this goal requires building a tool that is designed specifically to handle the task of annotating a video. Once developed, the usability of the developed tool would be evaluated and compared to that of other annotation methods; taking into account different aspects of the note-taking experience using them, such as the nature of the method, and the effect of the learning material (i.e. educational video) and its suitability for the annotation method. The tool to be developed will be called NotesKB (Notes Knowledge Base), and the other considered note-taking methods are a simple pen and paper $(\mathrm{PnP})$ and the Microsoft OneNote.

To achieve usability, our tool should first enable students to record their perceived knowledge from online materials in a certain way that reflects their thoughts, in a cohesive and an active learning space that requires minimal cognitive load from the student. Another requirement is that the design should accommodate ontological entities to be included in the form of semantic annotations that make use of some of the Linked Open Data (LOD) services, such as DBpedia. By analyzing the student's behavior annotating educational videos using a semantic-aware application, this thesis research is set to contribute to the research fields of Human-Computer Interaction as well as the field of e-Learning. For a general overview of the study, please refer to 
Figure 1.1. The outer ring represents tools and the inner rings convey the theoretical bases for the tools.

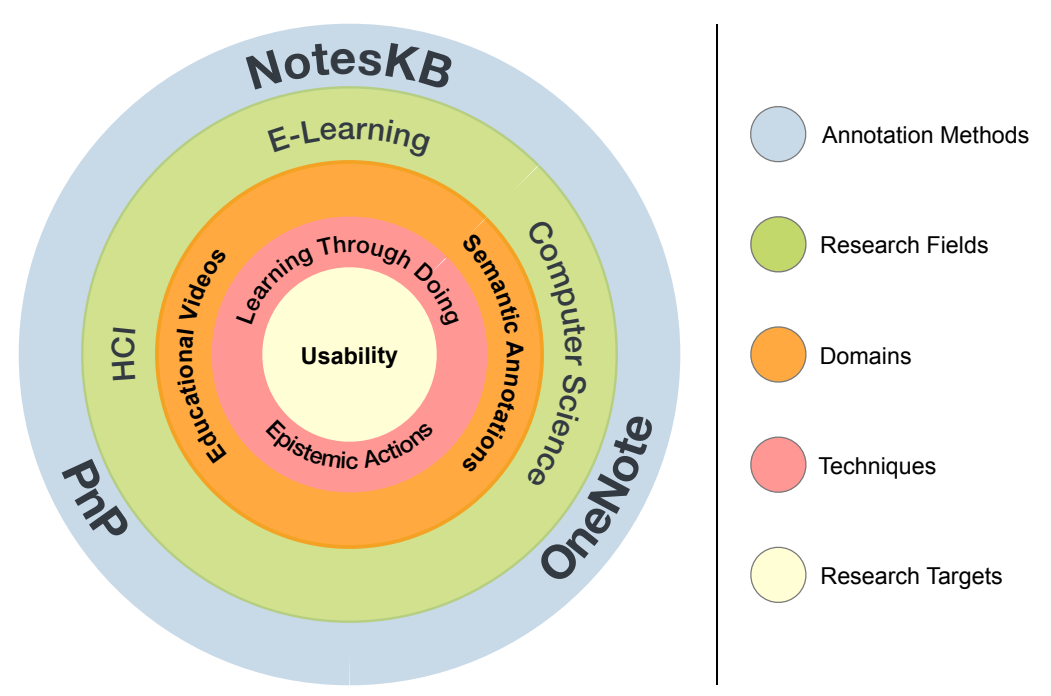

Figure 1.1: Overview of the different aspects of the research.

\subsection{Research Questions}

- What design principles should be effective for a tool like NotesKB?

- What is the usability of using PnP, OneNote, and NotesKB to annotate online educational videos?

- What are the factors that affect the note-taking experience of online educational videos the most?

- Does annotating style affect annotation outcome? 


\subsection{Proposed Solution}

NotesKB would be a web service that supports the authoring of and organizing of semantic annotations taken by the learner of online educational videos. Users (i.e. learners) would interact in the environment through an extension in the Google Chrome browser and could navigate the environment through an interactive singlepage Web application. The system would allow users to annotate, hashtag, watch and control playing pace of educational videos offered on the YouTube website. Also, it would give the user to chance to further discover and learn more about an input hashtag and linking it to a specific Wikipedia article.

\subsection{Specific Objectives}

The primary objective of this thesis is to provide an effective and efficient user experience to students using the tool to annotate multimedia online learning content. Beyond establishing this goal, this thesis aims to investigate the other factors involved in the activity of note-taking.

1. Building a notepad tool (NotesKB) as an extension of a web browser to facilitate the task of annotating learning resources on the Web.

2. Conducting a study with university students to assess their satisfaction using a semantic annotation tool and compare it with other predominant ones to take notes of online educational materials.

3. Reviewing the students' reflections and insights on their experience using the three annotation methods then classify them based on themes.

\subsection{Outcomes}

The building and design of the NotesKB tool is intended to: 
- Personalize the note taking experience by enabling students to channel their thoughts along with perceived knowledge in the annotations and categorize them while typing.

- Produce a machine readable information by incorporating ontologies in the LOD cloud into taken annotations.

- Keep records of the time when different subjects were first learned as well as some pointers to the location they come from.

- See it to compare an annotation method to another and the various factors involved in doing such comparison.

- Offer a venue to learn by constructing objects demonstrated in the incorporation into studying notes various types of annotations and linking their hashtags semantically based on LOD's ontologies.

\subsection{Thesis Structure}

This thesis project has six chapters in total, this included; and they are structured as the following:

- Background: in this chapter, we identify the key theories and concepts we based our work on.

- Design and Implementation: this chapter lays down all of the various elements, tools, and plans involved in developing the NotesKB tool.

- Methodology: in the Methodology chapter, we specify the testing methods we will use to conduct our exploratory and usability testing work.

- Results and Discussion: all of the results gathered from conducting our examination will be explained and discussed in details in this chapter.

- Conclusion: in this chapter is where we conclude our thesis and include a summary of our key findings from the study along with the prospect of the work.

Throughout the thesis the term "We" refers to the author, Abdulrahman Alamoudi, with the advice of thesis supervisors. 


\subsection{Motivation}

1. Knowledge acquisition and learning about new things have always been a personal interest. In the midst of the unprecedented growth of data witnessed nowadays, we intend through this project on understanding the mechanism and the necessary components involved in designing an instrument meant to help users capture new meanings and making sense out information.

2. Finding a better way to review and retrieve old notes collected throughout the years studying in the university. The experience of reviewing these notes is troublesome as it requires using multiple software applications with limited interactive features.

3. A first step toward building a personal smart book that can answer the user's questions about its content. 


\section{Chapter 2}

\section{Background}

This chapter reviews e-Learning from different perspectives in an attempt to give a generalized overview of the various factors and challenges currently facing it. It is mainly focused on exploring the relation between e-Learning and the Semantic Web (SW) and how each could add to the other.

The chapter starts by defining what constitutes human knowledge followed by a few of the most acclaimed theories in the field, explaining how people obtain and learn new insights. Following that is a summary of how the SW and its common standards are contributing to making more data ready for consumption by having their semantics more accessible to eventually facilitate the automation of the production of new knowledge that is shareable. Finally, we quickly browse some of the semantic annotation tools and projects and explore some their capabilities; also their limitations.

In the light of the sharp increase of Web technologies and information available to the public, there are now over 3 billion users around the globe with unparalleled access to information more than any group of humans before [8]. Internet usage is rising every year, and it is expected to continue to do so in the future as we are becoming increasingly dependent on the vast amount of data we consume [58,67]. The problem is that the majority of data on the Web is represented in many different forms; consequently discovering and integrating become more challenging to do [102]. However, with representing them in a standard way using Semantic Web technologies (e.g. RDF, OWL, and SPARQL) and standards, that could change.

This growth of Web usage has also led to an increase in e-Learning usage too. By e-Learning, we simply refer to "the use of the Web for learning" [57]. According to Docebo's forecast report on e-Learning trends for 2014-2016 [27], the number of 
students of higher education institutions in the United States that are enrolled in at least one online course has increased approximately three times since the year 2011. The growth is a worldwide phenomenon and is expected to increase even more [27]. E-Learning environments are gaining more favorable perceptions from educational institutions and students alike, and they are increasingly seen as viable means to achieve an ongoing learning experience for all those involved [2]. Additionally, the focus of many e-Learning researchers and practitioners is principally the idea of building a smart learning environment that is capable of generating personalized feedback to the student, and adapting to his/her needs at an individual level [59, 2, 102, 72]. According to Bloom, personalized learning (e.g. one-on-one tutoring) is considered to be the best for learning $[13,102]$. Integrating adaptive learning tools would help bring about developing data-driven learning environments that in turn would assist in making tailored content to students' needs possible [2]. Although realizing such a goal is still far from being reached, advancements in technology (computers, networks) science (artificial intelligence, psychology) and understanding the conditions of human learning (cognitive science, human learning) are gradually closing the gap [102].

However, the future of e-Learning is not solely determined by technological advancements, but it includes other philosophical, educational and even artistic ones too [72]. Reasoning and evaluating the learning outcomes of students is a broad subject and goes beyond the scope of this thesis. Nevertheless, constructing a student model is one critical component of the process of reasoning about those learning outcomes. The model is meant to represent the student's current knowledge and learning needs that then the e-Learning system should take and base the instructions according to it [102]. Building a student model is difficult due to the subjective nature of learning as an activity; its outcomes are determined by various factors such as level of expertise, cognitive ability, age and learning environment (i.e. context) $[14,102]$. Not only that, but parsing a student's input semantically represents a technical issue as well [102]. 


\section{$2.1 \quad$ Knowledge \& Learning}

Defining what constitutes knowledge has always been a debated subject[93]. There is a whole philosophical field of inquiry dedicated to finding answers to this very question, known as epistemology (or the theory of knowledge) [74, 93]. There is more than one way to classify and categorize knowledge [94]. One popular classification of knowledge is stated in the revised version of the Bloom's Taxonomy of learning objectives by Benjamin Bloom ${ }^{1}$. It was first introduced in 1956, but in its revised version of 2001 the taxonomy included four categories of knowledge (sometimes called Knowledge Dimensions): a) Factual Knowledge, b) Conceptual Knowledge, c) Procedural Knowledge and d) Metacognitive Knowledge [4, 72].

A different perspective of looking at perceived knowledge can be through its nature and subject matter, sometimes known as domain knowledge. Domain knowledge can be described as a representation of an expert reasoning about a discipline and a particular area of interest in some context [94, 102]. Domains differ in complexity and structuring, and sometimes they can overlap and be difficult to analyse. However, they are sometimes identified with the following simple categories [102] illustrated by the diagram in Figure 2.1:

- Problem-solving domains (e.g. mathematics problems, Newtonian mechanics, and most of Computer Science): such domains often have straightforward and correct answers to posed problems. These domains are illustrated in the top-left corner of Figure 2.1, in the clear and the well-structured area of the diagram.

- Analytic and unverifiable domains (e.g. ethics and law): such domains are characterized by lacking absolute measurements, right/wrong answers or reliable empirical evidence to establish claims in the field. These domains are positioned

\footnotetext{
${ }^{1}$ It is worth noting that there are some other classifications of knowledge than the stated Bloom's Taxonomy. A different classification relies on the factive sense of the word "know" as a base, and it breaks knowledge down into two (sometimes three) categories: a) propositional knowledge (known as "know-that"), b) ability knowledge (known as "procedural knowledge" or "know-how") and c) acquaintance knowledge [74, 93]. We are mostly concerned with the first one (propositional knowledge), which is the ideal one that we normally tend to possess and perceive in our personal notes. Its manifestation comes in the form of a declarative statement that holds a value of either true or false, but not both at the same time [74].
} 
at the center of the diagram of Figure 2.1.

- Design domains (e.g. architecture and music composition): the learner's goals in these domains tend to be centered around novelty and creativity, not solving problems. Such domains are located at the far bottom-right corner of the complex, ill-structured area of the diagram.

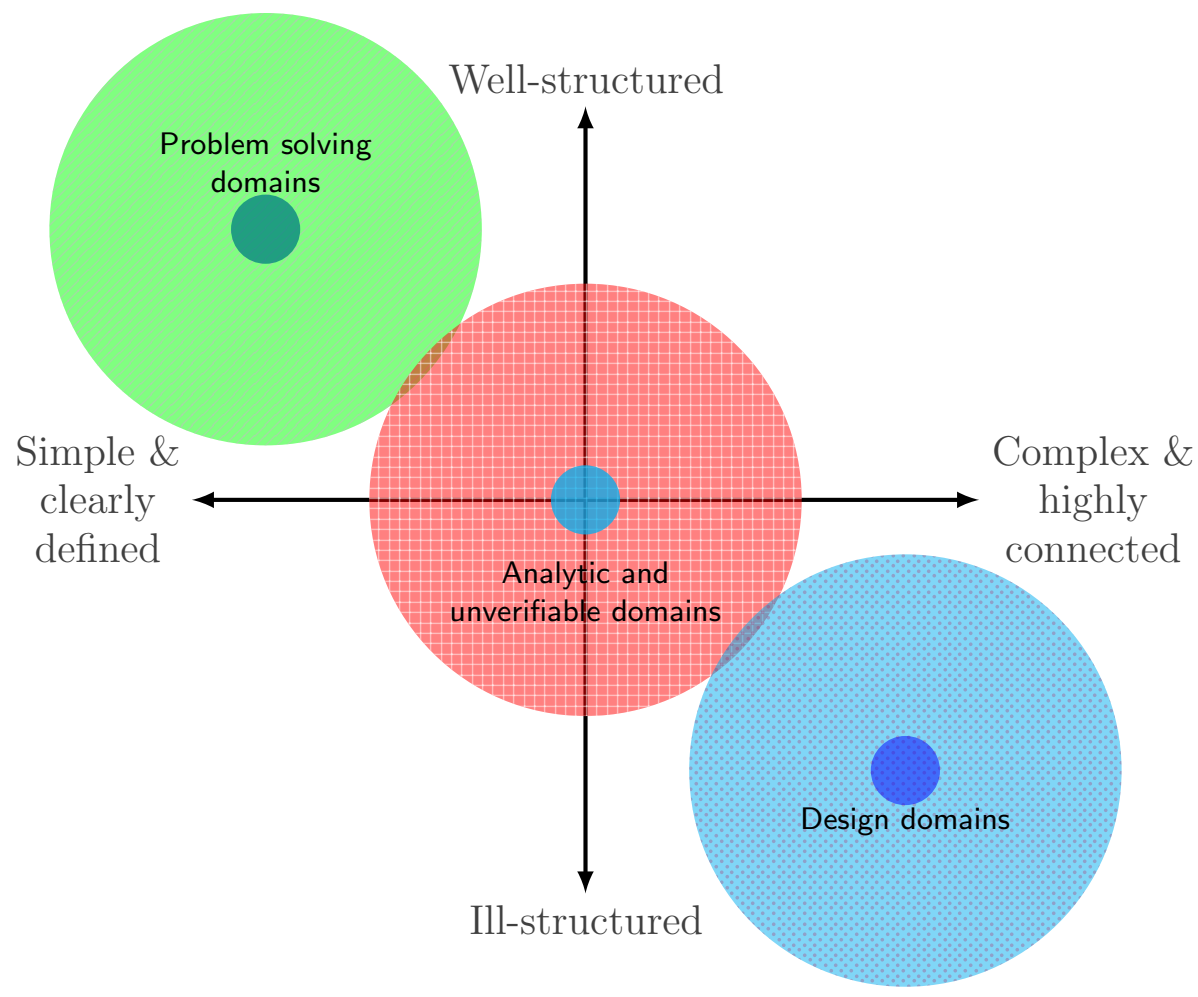

Figure 2.1: Categories of domain knowledge.

\subsubsection{Learning Theories}

There is no short answer to the question, how do humans learn? It is a heavily debated issue. However, here we will try to shed some light on the current influential theories that have repeatedly been adopted and examined by different schools of thought.

As mentioned earlier, learning is a subjective activity. So, the decision of using one theory over another as a guiding principle when designing a learning interface may result in a noticeable impact on the learning outcomes. That is to say; e-Learning 
applications will differ as a consequence of choosing one theory over another. The theories we review are Behaviourism, Cognitivism, Constructivism and Connectivism $[72,102]$. As it turns out, the Constructivism and the Connectivism theories are the theories that most suit our purposes for this study and align well with our research goals.

\subsubsection{Behaviourism}

This approach embraces a narrow concept of "science;" as the practitioners of it are solely engaging with observing overt actions (i.e. behavior) that can be empirically measured paying little to no regard to the inner operations of the mind [72]. It relies on positive and negative reinforcements as techniques. It also uses behavioral conditioning that assumes behaviors associated with comfort and pleasure are more likely to recur than those are not. Measuring, predicting and manipulating patterns of behavior in a learning environment depends on the educator intentionally providing explicit and outlined stimulus; that follows a stimulus-response pattern. In practice, though, this theory applying mostly to e-Learning systems with the main focus on memory and recall tasks. In such designs, computers tend to do most of the controlling and planning of UI elements as opposed to the user [102].

\subsubsection{Cognitivism}

From education and cognitive science, a schema (or a mental model) refers to an abstract existing mental framework or structure used by the learner to organize and categorize knowledge [72]. A schema (or a mental model), in the context of an e-Learning system, represents the expectations and thoughts that the user upholds about the system as they explore it; regardless of whether they match the essence and reality of the system or not $[9,72]$. Among the different theories, we found the Cognitive Load Theory (CLT) to be most relevant to our work as it is well established and it is capable of providing a theoretical framework to understanding multimedia learning. It addresses the limitation of the working memory of the learner, mostly from 
an instructional design perspective [70]. According to CLT, learning is described as a process of schema acquisition [56], and a learner's mind acts as a computer in which information is stored and retrieved in a certain way [72]. The cognitive load represents the amount of information one's mind attempts to process at a given time; in which each person has a limited capacity of [72]. The aim of the educator (or e-Learning system) then is to minimize that load on students. There is a direct relation between the cognitive load and the learner's performance, in that the less the learning task requires from the learner's working memory, the easier it would be for him/her to acquire new schema. For example, when low-performance students use challenging interfaces or perform difficult learning tasks, that would disrupt their performance even further [69].

\subsubsection{Constructivism}

"Learning is building," is the main premise of Constructivism [72]. It is the most evident theory that embodies e-Learning nowadays, with technologies that enable group discussion and wikis, discussion forums, chat rooms, and so forth; where socializing is integral to the learning task. This theory proposes the following:

1. The representation and construction of knowledge are based on personal preferences and are subject to the interpretations of an individual. Thereby, knowledge does not necessarily conform to reality; rather it differs from one person to another $[52,72]$.

2. Past experiences and prior knowledge are the bases in which humans establish on their newly acquired knowledge. One may think of knowledge as a dynamic, evolving, adaptable building that is in a continuous state of construction by the learner as well as by his/her surroundings (e.g. society) [30, 28].

3. Learning requires social interaction, and the knowledge gaps of an individual to be made explicit. Hence, the role of an educator to design a learning space that allows for such interaction to take place $[52,23]$.

In the constructivist view, context plays a role in the knowledge acquiring process. 
So, knowledge is formed when learners are actively working with their environment and interacting with it [14].

\subsubsection{Connectivism}

In this digital age, staying up-to-date with changing online content is difficult. Using technology to make connections with that content will help one to learn. The connectivism approach explains that making connections between different parts of learning materials is what constructs knowledge. Also, making those connections is considered to be the important part of learning, more important than the quantity of knowledge acquired from those sources; since knowledge would always exist outside of the learner. Connectivists argue that learning is a nonlinear process and it is located at the center of connection building. Understanding ecology helps in understanding the complexity of a successful learning environment too. So, our experience interacting with an interface is different from all other users. Therefore, designing a specific experience is not achievable under this theory; which could be taken positively considering that the learner's interaction with the system would make way for a personalized learning to occur [72].

\subsubsection{Related Concepts}

\subsubsection{Active and Passive Learning Hypothesis}

According to the Active-learning Hypothesis, a learner who is engaged closely interacting with the material learns more than who does not. It is based on the constructivist models of learning. During an interaction, the user would have full control over information delivery. However, the Passive-learning Hypothesis suggests that there is no significant effect on the learning outcomes when the learner uses an interactive system. Thus, the learner's task then is to simply memorize information that was transferred to him/her by an expert [30]. 


\subsubsection{Learning by Doing}

As its name indicates, Learning by Doing refers to a learning approach in which learners manipulate the material they are trying to learn [75]. That is best exemplified in modern software applications designed for students to learn different concepts by programmings such as Scratch, Alice, and the Cargo-Bot app; in which a learner in such environments is expected to learn computation and mathematics from being involved in the programming task [72]. Other examples of this type include Learning by Searching [105] and Learning by Creating [24]. Upon examination, a connection between constructionism and this type of learning can be established [24, 72]. Moreover, it can take part in both formal learning (typically delivered in an externally structured curriculum by a learning institution) and informal learning. An example of an eLearning design for formal learning includes structured multimedia course materials with laid down objectives. For informal learning, a designer may design an environment for a collaborative space to exchange skills among the workers.

In a learning context, the Montessori method is used to actively engaging elementary school students in the learning process. By using the Montessori blocks, a student can understand mathematical concepts (e.g. arithmetic) by engaging with them and moving them around [50]. Another inspiring example of this type of learning is exemplified in the cyclical learning process that children in kindergarten typically go through, as illustrated in the Figure 2.2 [72]:

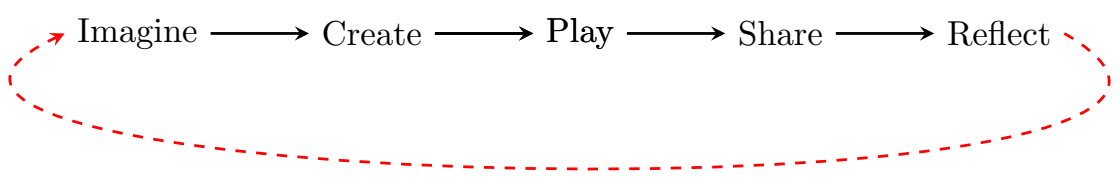

Figure 2.2: Learning by Doing Cycle.

From that, we learn that keeping the learners mindful of the tasks they are performing is important to the learning process; as that would open the door for them to gain new understanding about those tasks [76]. 


\subsubsection{Epistemic Actions}

Good learning experience designs are supposed to encourage and allow exploring of learning content; such acts are known as epistemic actions. According to Ware [99], an epistemic action "is an activity intended to uncover new information." It is the type of action that involves searching for a solution or a strategy in a different environment to execute a task. This type of action helps facilitate cognitive work of a person. It is considered different from a pragmatic action, which is strict to accomplish a task in a direct way [50].

\subsubsection{Learning Control}

Creating a new interactive visualization system that boosts learning experience requires a deep understanding. In other words, we are obliged to investigate how much control a user should have to be able to guide the learner's focus toward the learning material rather than the interface. In the literature, we found three types of controls a user has over instructional content in a multimedia learning environment. Those controls are $[28,72]$ :

- Pacing: different learners have different speeds of comprehending content presented in a video or other forms of multimedia. Consequently, it is necessary for the user to be able to control how media is played by starting, stopping, pausing or replaying. This is also known as control over information delivery, coming from the ability to speed-up/slow down information delivery.

- Control over content: having the ability to adjust the amount of information displayed to the user (e.g. the number of search results) as well as its quality (e.g. present in a selected units).

- Control over representation: having another view that represents the same entity in a different form. Such control allows learners to access information in ways that align with their mental models.

A general rule of thumb when designing a learning experience is that novices typically 
should have less control, and more guidance, whereas experts need to have more control and less guidance [72].

\subsection{Semantic Web}

The term Semantic Web was popularized in 2001, in an article in site Scientific American [10]; among the authors of that article was Tim Berners-Lee, the inventor of the Web. In the article, an early vision of the SW was articulated in which it builds on top of the original Web and not replace it. The idea emphasizes sharing information on the Web based on its semantics, and of the means to do that is to transform the current text-based Web into one of the Linked Data (i.e. "Web of Data") [96, 85]. The vision of building the SW is attainable, Berners-Lee thinks [85].

For e-Learning in the SW, there a great potential for it to transform learning and make it more personal (Personal Web) $[102,59]$. The main focus of the Personal Web is to customize the Web according to one's preferences, in this case, the learner. When observing the deployment of SW technologies in general, and in learning environments in particular; three trends/aspects appear to be central [59]:

- Knowledge representation: expressed in metadata or structural language.

- Knowledge generation: through sharing and collaboration.

- Personalization of the gathered knowledge: inferring and predicting personal preferences based on prior gathered data about the user.

Together with the elements discussed above, illustrating and giving some context about the SW technologies involved is important; some of them are as follows:

- IRI/URI (Internationalized/Uniform Resource Identifier): which can be thought of like the naming mechanism for objects and relationships (refer to Section 2.2.1) in the Web, paving the way to reason about them. Providing a naming mechanism is essential given that for something be to be named, it has to be identified first, henceforth reasoned about [85, 73]. IRIs (or URIs) are considered to be the building blocks for RDF (see below). The difference 
between IRIs and URIs, is simply that an IRI permits for a wider range of Unicode characters; thus a more general than a URI [51].

- RDF (Resource Description Framework): a syntactical convention and simple data model used to describe a triple; which consists of a subject, a predicate, and an object. It is a general-purpose language used to declare and create statements $[51,102,73]$. When modeling data using RDFs, each element of the triple is supposed to have an IRI as a value; except for objects which can have a literal value instead [51].

- RDF (RDF Schema) and OWL (Web Ontology Language): with subtle similarities between both languages, they serve the same purpose, that is to create ontologies. However, they differ in their capabilities, as OWL is more expressive when it comes to specifying classes than the RDF Schema [39, 104]. Using such languages, one can define the 'married to' relationship to be bidirectional. For instance, the statement "Jane is married to John," would be equivalent to "John is married to Jane" [73].

- SPARQL (SPARQL Protocol and RDF Query Language): a query language used to search and retrieve for RDF graph content that could facilitate the discovery of existing relations between different ontologies on the Web [104].

With the mentioned technologies, e-Learning system developers could build metadata and information rules that suit the learning objects of a virtual learning environment. According to Martin et al. [59], a learning object "represent the basis for content delivery and exchange in an educational environment;" which has two components, content (e.g., images, text, videos, and simulations) and interface exemplified in the metadata. The essential role of the interface, thereby metadata, is to best describe the meaning of the learning content, so it is easy to be discovered, reused, and managed by search engines and other semantic-aware applications. To build metadata with such capabilities and high readability, a developer could utilize some of the resources available in some online repositories such as Schema.org (www.schema.org). 


\subsubsection{Ontologies}

The term ontology is an old philosophical term used to describe the study of being, or what is real $[6,38]$. From a technological standpoint, however, the term has a different meaning. A technical definition by Gruber [37] is frequently used to define the term, and it denotes ontology as "a formal, explicit specification of a shared conceptualization" [90, 94, 91, 68, 46, 104]. The word 'conceptualization', generally refers to a phenomenon that structures a piece of reality and 'explicit' means that their use constraints are well defined. Nonetheless, there are some who oppose that definition, such as the case of Guarino and Giaretta [38]. They suggest that the word 'conceptualization' in Gruber's definition refers to the word's extensional notion, while it should be to its intensional one ${ }^{2}$.

In general, an ontology has two properties: a) it shares a common understanding about a domain among interested entities (e.g. people, machines) and b) it enables reuse of domain knowledge [86]. Ontologies are designed to capture both the structure as well as the semantics of an information environment, thus reach a consensual knowledge. Using semantically coded data extends far beyond a specific domain, as ontologies are more flexible and can be used in different domains; for example, in education, business services, health, media, and technology. A system benefits massively from adding semantics to data as it facilitates maintaining the system knowledge, easily integrating it with other data, are also the ability to reuse it and share it elsewhere [18]. Importantly, the use of ontologies is one of the several approaches for modelling and manipulating knowledge bases; mostly applied in educational and environmental related systems $[91]^{3}$. Each ontology consists of at least the following three elements;

\footnotetext{
${ }^{2}$ A good example from The Penguin Dictionary of Philosophy [60] which illustrates the difference between extensional and intensional contexts. Consider this statement: 'Socrates is wise.' Apart from 'Socrates,' the remainder of the sentence ('... is wise') forms its context. The difference between extensional and intensional becomes evident when replacing the expression of 'Socrates' by another, for instance 'The husband of Xanthippe.' An extensional occurrence of the phrase 'Socrates' happens when it is bound to the same truth-value as another expression (i.e. 'The husband of Xanthippe'). On the other hand, in an intensional context (e.g. 'P believes that Socrates is wise'), replacing 'Socrates' by another expression that has the same extension need not have the same truth-value.

${ }^{3}$ Please refer to Thomas et al. [91] for further reading on other approaches of knowledge bases modeling.
} 
a) classes: (concepts used to in a given domain), b) relations (describing how different concepts relate to one another) and c) instances (real world phenomena) [91]. Moreover, ontologies tend to be classified in the literature based on the type of knowledge they convey and its level of generality. So, an ontology may fall into one of the following categories $[91,90,81]$ :

- Application ontology: is the least general type of ontologies, as it only gathers knowledge required to model a particular domain. Thus it is not reusable.

- Domain ontology: aimed at clarifying and unifying terminological representations of concepts used amongst members of a specific community; this type of ontology captures all knowledge relevant to a particular domain (e.g. medicine, chemistry, philosophy).

- Generic ontology: this type of ontology can be applied across domains; thus they are referred to sometimes as top-level ontologies and as core ontologies. Examples of generic ontology include time, space and mathematics. Generic ontologies tend to be applicable in many technical domains.

- Representation ontology: unlike the previous types, this type conveys representational entities without stating what should be represented; which in turn means they are not confined to any particular domain. This type of ontology is used when describing the core essence of what class, a relation or a function means for instance.

The Domain ontologies will be the prime focus of our study, due to their applicability in different areas of e-Learning, such as student modelling and knowledge domain construction [91]. For a domain ontology to be 'usable,' one has to meet the following criteria [63]:

- Coverage: concerned with how far does the ontology being used/made for a certain domain, identify all of its relevant concepts as well as how are they related to each other (i.e. type of relationship).

- Consensus: concerned with the level of agreement among experts and specialists in a given domain on several issues linked to the making of the ontologies.

- Accessibility: concerned with the ontology's ease of access. That includes, for 
instance, the ability to share a given ontology on the Web.

As mentioned earlier, the use of SW technologies in the e-Learning system, with ontologies included, is found to be useful for searching and information retrieval. The mechanisms to achieve that is done by enhancing the structuring and the organization of the content, as doing that is considered critical to building an effective e-Learning system. Hence, some of the common purposes behind building/using an ontology in an e-Learning context would fall into the following categories a) curriculum modelling and management; b) describing learning domains; c) describing learner data (e.g. profile data) or d) describing e-Learning services [41].

\subsubsection{Linked Open Data (LOD)}

In this quest of creating a Web of Data, a standardized set of principles and best practices to publish data to the cyberspace is important. This led to the introduction of Linked Data [95, 12, 104]. Linked Data is not only concerned about the accessibility of data sets but also about the relationships between them. Applications built using Linked Data's best practices tend to have higher quality, and are more scalable [87]. Although it has established itself as the de-facto standard for sharing data on the Semantic Web, its adoption rate by learning enhancing technologies is still low [26]. Using Linked Data is intended to amplify applications' abilities to incorporate available knowledge and share newly created ones. It is worth noting when a service decides to produce its data according to the Linked Data best practices, that does not necessarily mean the published data will be available to the public; services that serve such a purpose are part of what is called Linked Open Data (LOD). Datasets published through some of the cloud LOD services, such as DBpedia, Wikidata, or Freebase; provide the public with a cross-domain, open and free of charge access to them [31]. As we will see in Chapter 3, we relied on LOD ontologies retrieved from the DBpedia project automatically when linking Wikipedia articles to the student's notes.

Building applications using Linked Applications, implemented with the Linked Data

principles and best practices in mind, offers large potential in improving search 
efficiency and makes the problem of data interoperability less severe because the interpretation of a given concept is shared with different parties [19, 26, 35]. An example of datasets published using these principles is data.gov, by the US federal government. According to $\mathrm{Yu}$ [104], "The goal of data.gov is to increase the ability of the public to easily find, download and use datasets that are generated and held by the US federal Government. Linked Data principles are at this time the best practice guide to ensure the accomplishment of these goals."

\subsection{Semantic Annotation}

For at least a millennium, annotating published works has been an integral part of practicing and doing scholarly work; and to a certain degree, it has positively contributed to the collective human knowledge [101]. Clarifying and critiquing contents of published works, in some instances, has attracted the almost equal amount of attention to the original annotated work [3, 101]. A good analogy to illustrate an annotation, is to think of it as an arrow with a payload that points at a target [Tremback [92];hypothes.is.designersandgeeks:2013]. From that we learn that every annotation is expected to have three elements: a) an address, b) a payload, and c) a target. Where the address denotes the location in which the created annotation resides (usually via a unique URI); and the payload represents the contribution made by the annotator; and finally the target symbolizes the piece of content being annotated. Noting content can take different forms, and Whaley [101] classifies them into four types: a) annotation; b) comment; c) highlight; and d) bookmark. This classification is based on the availability of three components in each type; the components are (see Figure 2.3):

- Body of text input by the annotator;

- Target/URI to the segment of annotated content; and

- Selection that determines the start and end of a segment.

However, annotations in the traditional sense tend to be exclusively about the static content (e.g. text), and that is no longer the case. As audio and video contents grow, 
now there are tools to annotate those contents too (please refer to Figure 2.4). An example of that is the Web annotation service Genius, which was designed to help users create annotations for music lyrics to explain their meanings [71].

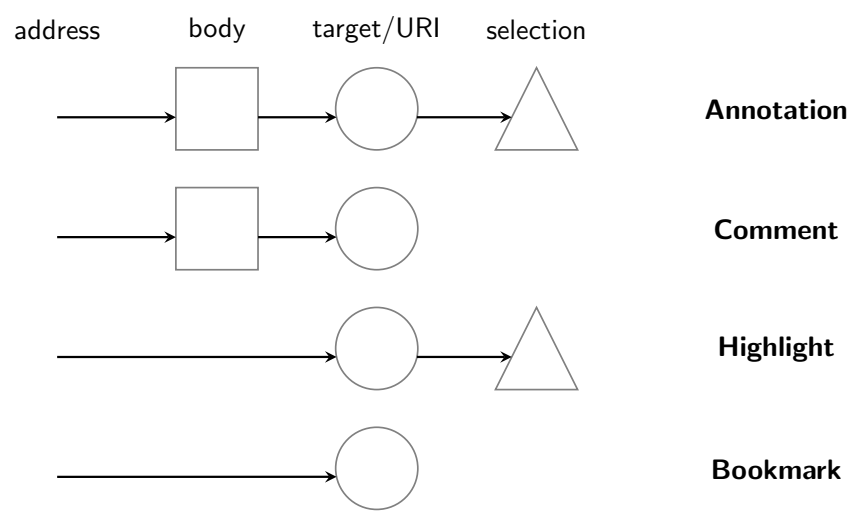

Figure 2.3: Categories of Annotations by Dan Whaley [101].

The diagram content is reproduced with permission from the author, Dan Whaley, from the presentation "Annotating Knowledge."

When it comes to Semantic annotation tools, that term usually refers to metadata that is defined by ontologies [100]. By appending ontologies onto text annotations, one sought advantage is to have these ontologies be linked to different ones that are related to them; either by inferencing or conceptual navigation. Over time, that would allow commonly agreed upon set of concepts in a given domain to emerge; unlocking the potential for computers to handle text smartly. Furthermore, it may contribute in directing the attention of the annotator toward predefined choices of previously structured semantics [88].

There are many annotation tools we came across while reviewing the literature. From this variety of annotation tools, we selected a few to represent that we thought are relevant to our work; especially those were made for the Web. Also, we tried to include those that are equipped with Semantic Web technologies the best we can; though there are some exceptions. Some of these surveyed projects include SemTube ${ }^{4}$ (a prototype

\footnotetext{
${ }^{4}$ http://www.semedia.dii.univpm.it/semtube/
} 


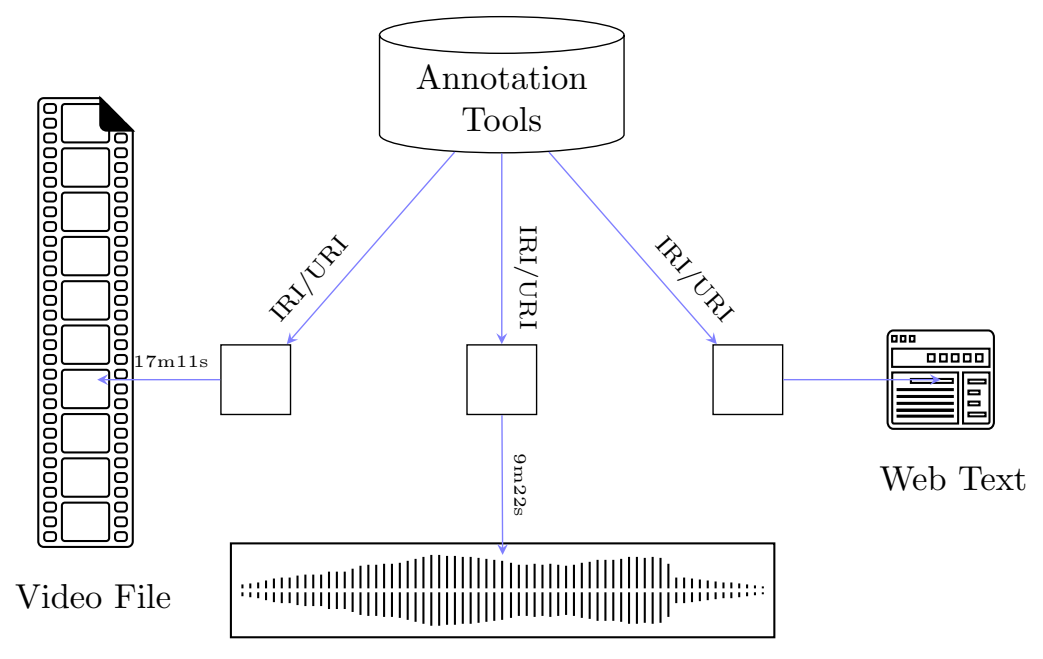

Audio File

Figure 2.4: Model of Multimedia Annotation Tools [101].

that later developed to a semantic annotation tool called Pundit) [35, 22, 36, 46], Annomation/SugarTube ${ }^{5}$ (designed in the Knowledge Media Institute (KMI)) [103, 46], Hypothes.is ${ }^{6}$, Annotorious ${ }^{7}$ (part of the EuropeanaConnect research project, and it used to be called Europeana Connect Annotation Suite[29]) [35, 46], Synote $^{8}[97,55,17,46]$ and the Open Video Annotation Project (OVA) ${ }^{9}$ $[45,16]$.

Academic projects (e.g., Annomation/SugarTube, OVA), and not academic ones (e.g., Hypothes.is), all share the same intent of enabling users to annotate multimedia content; however, the experience of using each one differs from one tool to another. We tried out some of these tools (e.g., Hypothes.is, Annotorious, and OVA), though not all due to either lack of admitted access (e.g., Synote) or availability (e.g., Annomation/SugarTube).

Based on our general observation, we identified some common characteristics and features that these tools share, summarized in Table 2.1. The point of this exercise

\footnotetext{
${ }^{5}$ http://annomation.open.ac.uk/

${ }^{6}$ https://hypothes.is/

${ }^{7}$ https://annotorious.github.io/

${ }^{8}$ http://synote.com/

${ }^{9}$ https://gteavirtual.org/ova/
} 
was to grasp the various aspects that affect the functionalities of these tools and in turn their usabilities. The first feature we examined was the tool's ability to support semantic annotations of some content. This is critical to our research project, because, as we explained earlier, we are concerned with the learning tools that help in making them. Another important feature we looked for was the annotation tool's ability to handle more than one type of multimedia, namely, videos, audio, and text. Also, we looked at how the tool could work and enable the user to annotate different material published on the Web. Moreover, we looked at each tool's ability to be expanded, and if it offers any application program interfaces (APIs) available to developers interested in adding new features to the tool.

Table 2.1: Summary of the reviewed annotation tools.

\begin{tabular}{lcccc}
\hline & $\begin{array}{c}\text { Support for } \\
\text { semantic } \\
\text { annotation }\end{array}$ & $\begin{array}{c}\text { Annotable } \\
\text { resource } \\
\text { types }\end{array}$ & $\begin{array}{c}\text { Used/Support for } \\
\text { content on the }\end{array}$ & Available \\
Tool & $\checkmark$ & Videos $^{*}$ & $\checkmark$ & APIs \\
\hline SemTube & $\checkmark$ & Videos $^{\dagger}$ & $\checkmark$ & \\
Annomation/SugarTube & $\checkmark$ & Images & $\checkmark$ & $\checkmark$ \\
Annotorious & (Undetermined) & Text & $\checkmark$ & $\checkmark$ \\
Hypothes.is & $\checkmark$ & Video \& & $\checkmark$ & \\
Synote & & Audio & & \\
& & Video & $\checkmark$ & \\
Oppen Video & & & \\
Annotation & & & & \\
Project (OVA) & & & & \\
\hline
\end{tabular}

Note: ${ }^{*}$ Exclusive to videos on YouTube. ${ }^{\dagger}$ Exclusive to videos on the Open University video repository. ${ }^{\ddagger}$ Probably images too.

Although students should see the benefits of using semantic annotation tools for learning purposes [100], there are still some gaps in the usability of such tools that need to be addressed. As Khan et al. [46] put it: 
“... we need to develop a collaborative video-annotation web application that allows its users to interact with multimedia content through a simple and user friendly user interface. Sufficient incorporation of semantics is required in order to convert annotations into semantic annotations ... SemTube has no support for theme-based annotations, and linking related scenes, events, themes and objects is difficult. Similarly, searching for specific event, scene, object and themes is not available."

Also, where most tools support videos, they do it in a way that can be described to be more a kind of a video segmenting than a video time-stamping. That in turns requires the user to assign the start and end of each annotation, which could consume extra time. As for the incorporation of semantics, in which LOD ontologies can be sourced through various services, users sometimes preferred using one service over another due to its familiarity to the users; as Yu et al. [103] found out in the case of DBpedia in comparison to other LOD vendors. Moreover, the input of the ontologies is usually done in a semi-automatic way, in that the tool search for similar ontologies from these services.

To recap, in this chapter we explored various theoretical elements and applied examples that are related to annotating videos semantically. We began by discussing knowledge and four influential learning theories. We assessed that Constructivism and Connectivism to be relevance, due to the simplistic views the two remaining theories, namely Behaviourism and Cognitivism, hold about the learning activity. In Constructivism, allowing the learners to be active during the learning process and facilitating the means for them to create and share their personal interpretations of the learning material is heavily emphasized [52, 23]. Thus, we reasoned that note-taking is a form of a building. For Connectivism, on the other hand, having dynamic parts of knowledge is considered to be the norm, and they are mostly influenced by external factors. From that, we reasoned that note-taking is a way of documenting connections between different parts of knowledge. As for semantic annotation tools, we found out that tools built according to LD's principles and best practices are more likely to succeed than those that are not. We also found that the usability of such tools is a 
significant hurdle to need to overcome. 


\section{Chapter 3}

\section{Design and Implementation}

This chapter discusses the design and implementation of the NotesKB system. It highlights and provides an overview of the design concepts and decisions involved in making both the back-end as well as the front-end of the service; with special attention to the technical and the usability sides of the design.

Building an engaging e-Learning experience is a challenging task. Simplicity is thought to be a key to achieving such a goal. Our intention is to design and build a tool that provides learners from different backgrounds, and different skill sets; the ability to learn from online video materials by annotating them. Although we will not seek to test for learning outcomes, the tool is expected to set the stage for more understanding of the relation between note-taking and learning.

\subsection{Proposed Design Basis}

This section describes the conceptual components, style, and processes involved in building the NotesKB system.

\subsubsection{UI Components}

There are three conceptual components that appear repeatedly:

- Collection: with a folder-like functionality as in traditional OSs, a collection serves as a hierarchical structure that contains all produced notes and organizes them. 
- Note: a set of annotations (defined below) that are related to a given learning video; in that each video has its own note file. A note can be thought of as an interactive document that clusters media metadata as well as the learner's thoughts in one place. Input annotations are ordered in chronological order, with the earliest displayed on the top of the document. For example, a user can create a new note to annotate a video about what is mathematics.

- Annotation: annotations are created and exist within a note, in that a collection of them are the basic building blocks of a given note. Besides text, an annotation may contain hashtags (defined below), and the user can assign it an annotationtype (defined below) too. Moreover, upon its creation, if the video being annotated was playing, a time-stamp (defined below) of the video will be tagged and becomes part of that newly created annotation. The four general components of an annotation are:

- Text: simply the words and content produced by the user addressing a specific point in the learning content. For instance, an annotation can include text such as "One approach to define \#mathematics, is through classifying it according to the questions it attempts to answer" [34].

- Hashtag: a label assigned by the user that ideally represents a proper noun. A hashtag is designed to be shareable and reusable across annotations and notes. Furthermore, a hashtag's label is the key that is used to search the DBpedia for a relevant LOD ontology; thereby having its text machinereadable as it would be semantically linked. A sample of a hashtag is the "\#mathematics" part from the earlier mentioned example.

- Annotation-Type: a text attachment that describes the nature of the annotation's content (e.g., text). This is thought to help further the user clarifying his/her ideas and reflect on them in real-time while typing them (see Appendix A for reference).

- Time-stamp: an interactive component that specifies the minute as well as the second in which the annotation was taken with respect to the video's playing time. 


\subsubsection{Note-taking Style (Annotation Style)}

Another important aspect of the design is the note-taking style. There are different styles of taking notes; some people mix drawings with text, some use different colors, and others write on only one side of a notebook. To create a cohesive note-taking experience, the annotation style has to match the capabilities of an annotation method. For NotesKB, we are interested in finding a style that is structured as a hierarchy, so that produced notes are easy to read and organize. Also, the style has to feel intuitive and look familiar to students. We called this style the Hierarchical Annotation Form (HiForm) style, and it has three characteristics:

- Annotations are written in a listed point-by-point form; in which the annotations are structured as elements of a list that are successively composed of other subannotations.

- Annotations include hashtags; which include the key ideas of a learning subject, such as the names of geographical places or historical figures.

- Annotations have annotation-types; as explained earlier, the type of an annotation describes the nature of the content.

\subsubsection{Wikification Process}

According to Mendes [61], "Wikification refers to the task of automatically marking up phrases with links to Wikipedia pages that describe those phrases. It involves both recognition and disambiguation challenges. It needs to recognize which of the words ... refer to an entity, while also determining which specific entity was the referent - in case more than one interpretation is possible." In our work, we recruited Wikification and applied it to the hashtag linking process (refer to Section 3.2.1.3); in which we convert collected textual hashtags and link them to Wikipedia articles they correspond to. Plus, all of this takes place within the NotesKB environment supporting 'in-context' note-taking. 


\subsection{User Interface Design}

From the start we had attempted to keep the design of NotesKB in harmony with the Learner-centered system design (LCD) guidelines [66, 40, 72]. LCD aims at enhancing the engagement of learners, and to do so, and it stresses three general requirements that each should be addressed in the design of the anticipated e-learning UI. They are outlined in the ability of the design to accommodate: a) the learners' continuous growth in knowledge and skills; b) their diverse backgrounds; as well as c) their motivation to learn. LCD's main objective is to engage a diverse group of learners, across knowledge domains and pedagogical approaches with changing abilities and needs to accomplish learning tasks/goals at different levels. It is worth noting that LCD is applied to children in general, although not necessarily restricted to them. Moreover, there is a distinction to be drawn between LCD and the User-centered system design (UCD). In UCD, designers seek to achieve faster/better execution of tasks and construct a general purpose design. Whereas, in LCD, "faster/better" of tasks execution is not an obligation; its main requirement is to assist and provide a level of support to the learning process of all learners. In LCD, the learning task directs the design and not the other way around [76].

NotesKB is designed as an online notepad application, that is part of the Web browser Google Chrome. Since the early stages of the design process, NotesKB was conceived as a browser extension. The reason for choosing the Google Chrome browser is because it is widely used, as well as the wide variety of APIs (Application program interfaces) and UI elements it provides. For example, the panels UI element function as external windows to the primary browser's window. At first, the design was made to conform and incorporate those panels; however, later in Chrome 54, the support for those panels was halted and discontinued by Google [15]. Consequently, the design had to change and adapt to the new reality. Eventually, it was decided that NotesKB would run as an entirely interactive website that occupies the space of a full-screen application. Moreover, to achieve some level of cohesion in the visual elements of the tool we modeled the UI after the principles of the Material Design ${ }^{1}$.

\footnotetext{
${ }^{1}$ https://material.io/
} 
In the early designing stages of NotesKB's UI, the design went through several phases. The first design phase of the UI was set on using the Chrome panels, which only offered a limited screen space, and that comprised a major constraint to our designing effort. By iterating through several design wireframes and mock-ups, to a large degree, we overcame the limited interaction space constraint that the panels imposed. However, the technical difficulties to implement such solutions loomed in. The biggest technical challenge we faced was finding a web-based UI library that functions within Chrome's panels and has the capabilities to accommodate dynamic interactions between different visual elements. The AngularJS Material ${ }^{2}$ has proven to be a perfect match for the job. Despite that, with the exception of Chrome OS, at the time Google Chrome stopped supporting panels in their browsers in all other OSs; most notably the three major ones (MacOS, Windows, and Linux). Thus, the whole UI design process came to a halt. Luckily, since the design was initially conceived to be web-based, migrating into a full website design did not consume a lot of effort (see Figure 3.1).

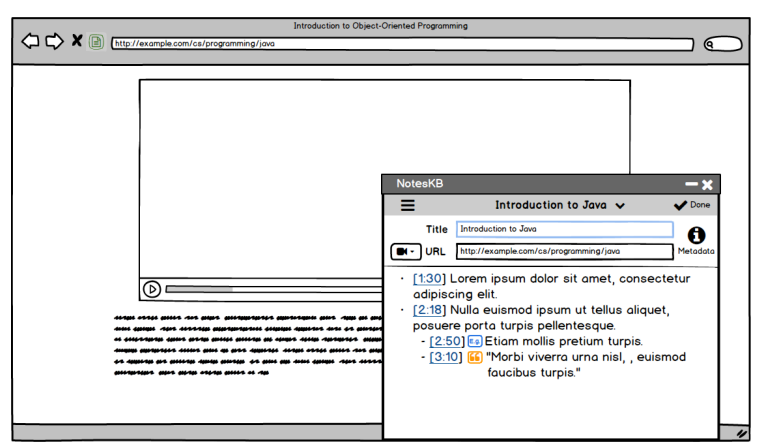

(a) Sketched wireframe

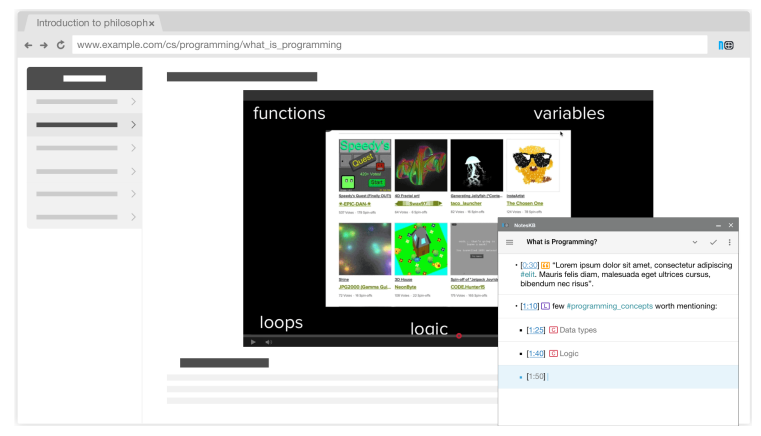

(b) Developed mock-up

Figure 3.1: Preview of NotesKB's early designs for the panel UI element in the Google Chrome browser.

To test the learner's engagement, we focused on online educational videos being the learning material. Enhancement of the note-taking experience involves speeding up the pace of video annotation. Accordingly, the design draws on some contemporary UI elements and techniques that facilitate that, even though some of them may not be that common in e-learning environments. For example, the use of 'hashtag' that is

\footnotetext{
${ }^{2}$ https://material.angularjs.org/
} 
commonly used in online social networks; also the placement of the text area at the bottom imitating typical messaging applications. The rationale behind that is to let users (i.e. learners) expand their knowledge over time by learning through doing and by taking epistemic actions all within the same (within-context) learning environment. With that in mind, NotesKB was designed to accommodate and even encourage such steps to be undertaken by the user for them to learn and explore more about a particular subject. An example of that is the searching mechanism incorporated into the 'Hashtags' tab which allows the user to search look for a Wikipedia article that is related to an input hashtag. Furthermore, searching for information comprises a part of the learning process too [105, 43]. Searching in NotesKB is offered within-context without the need to leave the learning context to look for more information.

\subsubsection{UI Components}

The front-end comprises of three main views:

\subsubsection{Library View}

This view is split between two tabs; the a) Notes List Tab and the b) Hashtags List Tab (see Figure 3.2). Selecting an element from either one would lead the user to that view's UI. The Notes List Tab is simply a sub-view that includes a list of all the notes that have been taken by the user at the time. The notes are ordered ascendingly based on their creation date, with the oldest appearing on the list's top. When a listed note is selected, the system would initiate a new note-taking session in the Notepad View and let the user resume their note-taking right where they had last saved their notes. Deleting a note can be done from this view as well. The other sub-view is the Hashtags List Tab, which was designed to serve the user with a list that includes all of his/her hashtags accumulated overtime in one place. The hashtags are listed in ascending order according to their creation date. By clicking on a hashtag, the user will be directed to the Hashtag Linking View. 


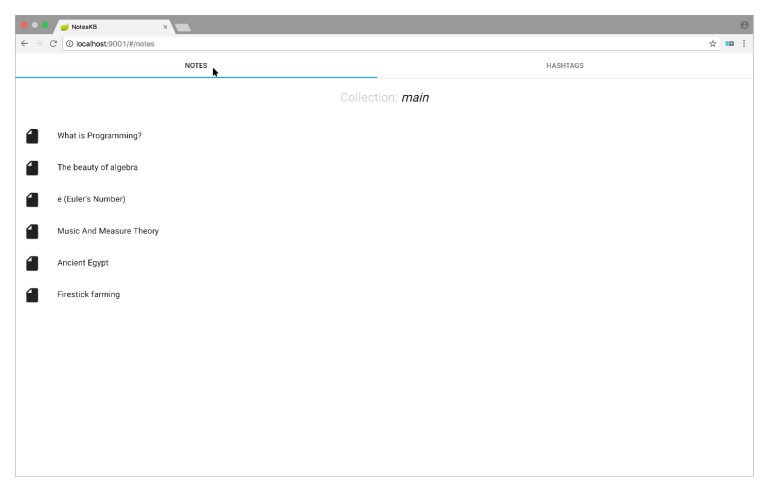

(a) Notes List Tab

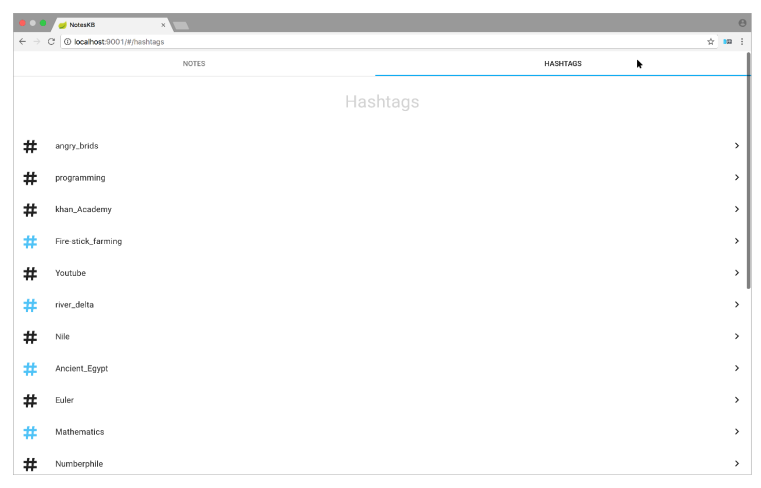

(b) Hashtags List Tab

Figure 3.2: An overview of the Library View view.

\subsubsection{Notepad View}

This is the view in which videos are viewed, and annotations are edited. It handles all activities related to note-taking. This view can be reached in two ways, either via the NotesKB extension in the Chrome browser or by selecting a note in the Library View. Once the NotesKB extension on Google Chrome is installed and launched, this view will be activated when the user presses its button that appears on the right top corner of the browser. Typically, the extension is activated while watching YouTube videos to ease the process of initiating taking notes. The activation would lead the user to a new annotation session in NotesKB (see Figure 3.3). The metadata (e.g. title, author, source link) of the video is also included upon activation to speed and ease the input process. The Notepad View consists of four major areas, they are, from top to bottom (see Figure 3.4):

- Controllers Area: controllers (e.g. 'Play/Pause,' 'Hide/Show' video) are placed on the top of the application.

- Media View Area: the area in which the educational video is displayed. The video in this area can be hidden whenever the user wishes.

- Annotations List: this list contains all the annotations taken by the learner of a specific video.

- Input Area: this area has two sides. On the left side of the area, the user chooses 
the appropriate Annotation Type of his/her upcoming annotation. On the right side, the user inputs the text of the annotation.

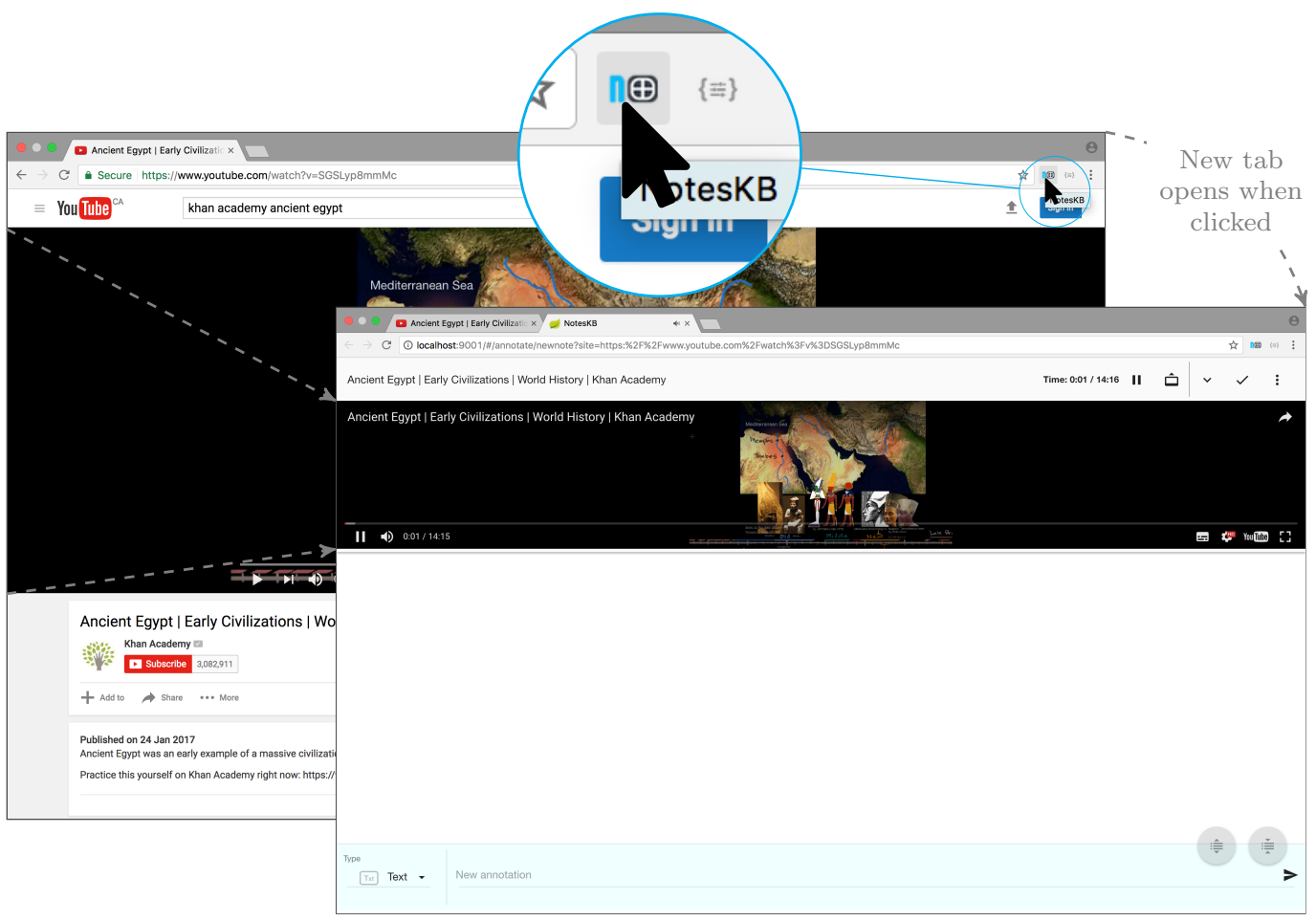

Figure 3.3: Initializing NotesKB via Google Chrome browser's extension. 


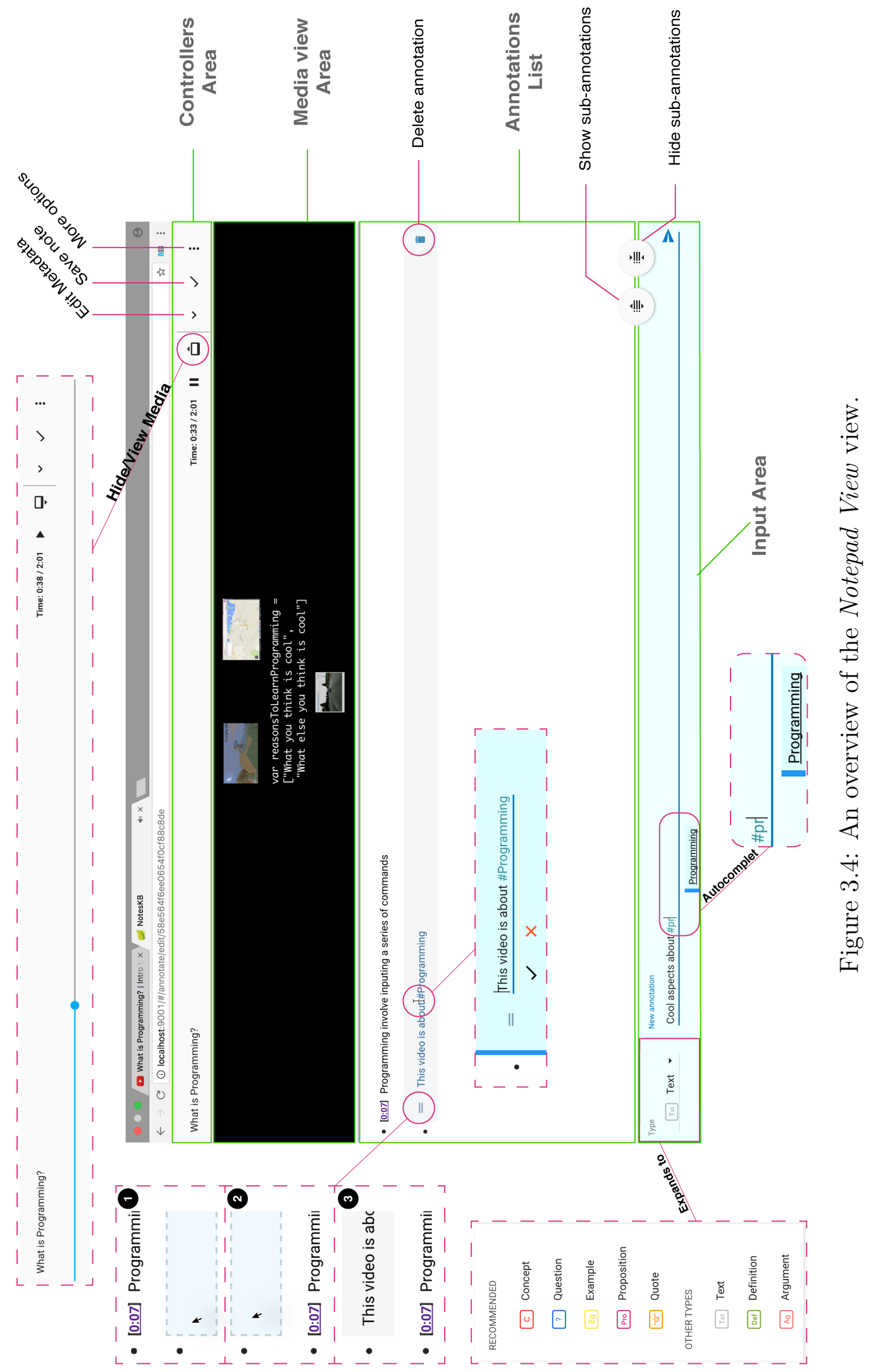




\subsubsection{Hashtag Linking View}

As part of the Wikification process, students are expected to include hashtags in their notes that denote the key ideas mentioned in the learning content; then linking those hashtags to related Wikipedia articles. As described, this linking process requires an intervention by the user, and this view is thought to help them in doing so. Also, the act of searching for a related article is considered to be beneficial to the student as it can be part of the learning process [105]. This view is meant to bridge the gap between LODs and text, all to make the users' notes machine-readable. The intention behind building this view is first to let users enrich their knowledge by carrying out some epistemic actions as they go through their collection of hashtags; then have them assign their hashtags to related LODs.
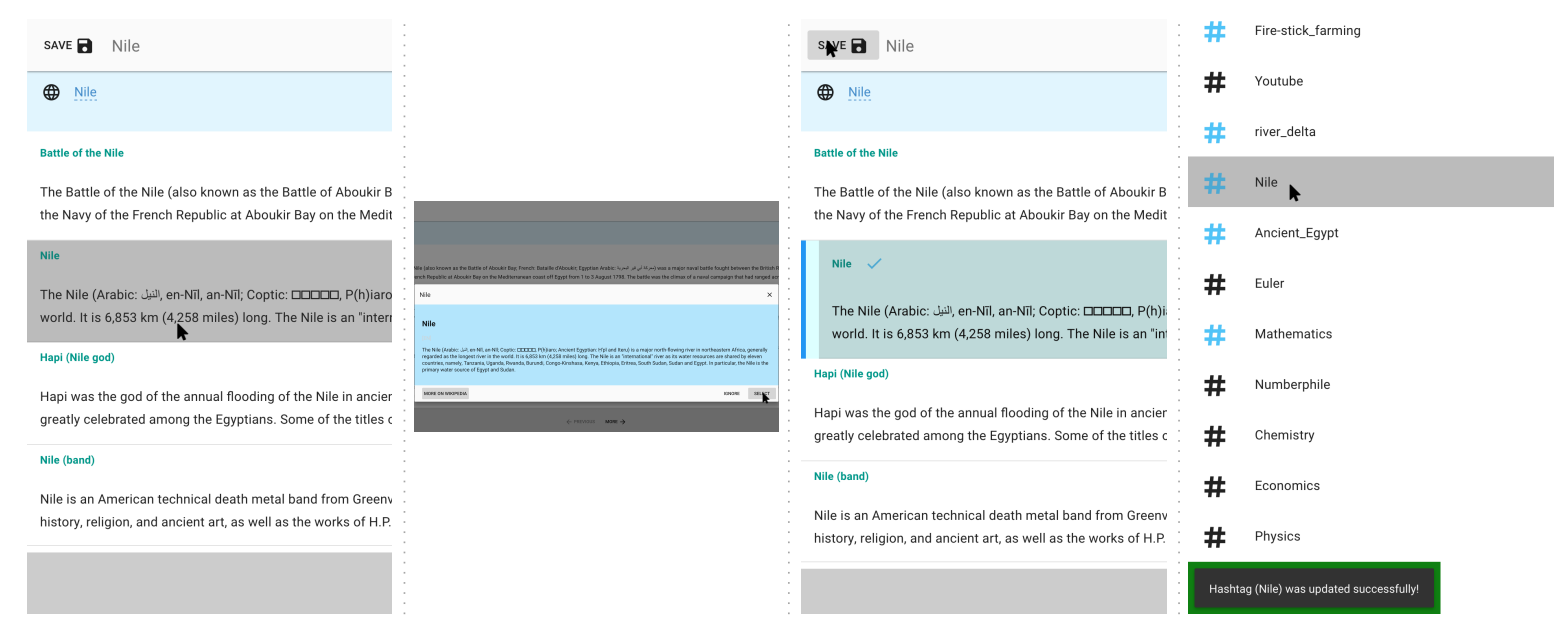

Figure 3.5: Steps involved in linking the hashtag \#Nile inside of the Hashtag Linking View as part of the Wikification process.

When a hashtag is selected from the Hashtag List Tab, the user will be led to another view with Wikipedia articles related to that hashtag's title are presented. The listing of articles involves querying the DBpedia repository and retrieving LODs that have a moderately high degree of lexical similarity to the hashtag's title. As a result, the user will read snippets or the full Wikipedia articles of a given hashtag and link the most suitable among them to be the selected semantic of the hashtag. To explain the 
selection process, when an article is clicked, the user will be prompted with a dialogue box that provides him/her with a short description of the article. From that dialogue box, the user can choose either to read the entire article (by clicking on the 'More On Wikipedia' button), select it, or simply ignore it. When a selection is made and saved, the user should notice that that hashtag's icon has turned blue signifying it is now linked. Please refer and follow the illustrated steps in Figure 3.5.

\subsubsection{Features}

\subsubsection{Pointing \& Timestamping}

This key feature is designed to help the user revisit a specific part of the video of a given time. The idea is that when the user enters a new annotation, the time of the video at the time of entering it would be attached to the annotation. For example, "[10:31] The \#delta" signifies that the video mentioned is something about the "\#delta' at the 10th minute of the video. This is thought to be important especially when the learner is reviewing or revisiting the notes.

\subsubsection{Creating and Connecting Hashtags}

Making the connection between various notes is another key feature anticipated to enhance the overall learning experience. Given the lack of connectivity observed in most of the annotation tools we reviewed, adopting of the hashtag idea from social networking and other blogging applications was thought to solve such predicament. One goal of this research is building notes that are semantically sound. Hashtags are thought to be the bridge between content and fast note-taking (i.e. annotation) practices. As an attempt to improve the user's overall experience, we sought to provide a quite simple cross-reference mechanism that suggests previously input hashtags based on their alphabetical similarity. Hinting hashtags to the user happens in real-time while the user is annotating a video. 


\subsubsection{Related Concepts and Techniques}

In this section, we primarily point out the design concepts and techniques used to develop the interface of NotesKB. With current web browsers becoming increasingly important in our daily lives, utilizing them to enhance the students learning experience was thought to be a worthwhile pursuit. As mentioned earlier, e-Learning is gaining a more favorable view among learners and educators alike. One reason for that might be linked to the existing tendency of students to prefer educational websites that contain videos, as Jakob Nielsen and Hoa Loranger have found out [72]. Our aim is to build an interactive digital learning environment that students find engaging. So, in the following subsections, we will review some UI design concepts and try to draw attention to their relations to e-Learning systems.

\subsubsection{Affordance}

Affordance is a popular concept that has gained a special attention after being addressed in Don Norman's book "The Design of Everyday Things." The notion of affordance has been revised recently by Norman and replaced by the term signifier in his latest revision of the book [65, 72]. Although signifiers are supposed to signal to user possible actions, unlike affordances, they specify how users discover those possibilities. From a designer standpoint, Norman argued that signifiers are far more important than affordances. An example of a signifier is the underlined text in a website, which signals linked content. Another example is buttons that appear to be clickable. These signifiers play a role in how the user interacts with a learning system and focus on the learning material instead of the interfaces [72].

\subsubsection{Cognitive Overload}

Interactivity has the risk of imposing an excessive cognitive load on the learner instead of reducing it [28]. One main challenge facing all e-learning interfaces is their need to reduce the learner's cognitive load. Some overload is caused by the design or the 
technology used, and that is known as the extraneous cognitive load. Unlike many of the technologies we interact with today, learning interfaces require more than simple mechanical entries that do not take much of our attention nor time to complete [28, 72]. Learning activities cannot be completed with no cognitive effort. Therefore, a designer has to put an extra effort to produce an interface that requires minimum interactions as possible to make the learner focus on the learning task at hand rather than the interface.

\subsubsection{Interactivity}

In general, for interactivity to occur, two fundamental conditions have to be met: (a) there are at least two participants interacting with one another, and (b) the existence of an element of reciprocity where changes happen on both sides. In the context of multimedia learning, Domagk et al. [28] define interactivity as a "reciprocal activity between a learner and a multimedia learning system, in which the [re]action of the learner is dependent upon the [re]action of the system and vice versa." That is to say, such environments cannot be intrinsically interactive, but they have the potential of engaging the learner in a dynamic relationship. Thus, emphasizing interactivity is not merely an attribute of the medium. Furthermore, a learning process can not be clarified by simply quantifying interactivity of its underlining system. That is to say, a high level of interactivity does not necessarily translate into an understanding of the learning material; in fact, it could lead to excessive extraneous cognitive load in some cases [28].

From a technological perspective, interactivity is a characteristic that is based on the affordances provided by the system, not the learner. That characteristic takes into account how content is delivered (e.g. the Web, video), what input devices were used (e.g. keyboard, touchscreen), and the UI features and styles utilized (e.g. hypertext, simulations) [28].

When it comes to the nature of the learning material and the UI, there is a need to distinguish between extraneous complexity (associated with instructional methods 
and interfaces) and intrinsic complexity (related to the student's primary learning task) $[72,69]$. The aim of the designer tends to be to minimize the former to help the student focus on the later.

\subsubsection{Learnability}

The term learnability should not be confused with learning interface design or learning outcomes. From a usability standpoint, the term is used to describe the user's ability and the easiness in which he/she could carry out operations with the minimal possible amount of instructions and time spent to learn how to carry them out [72]. Learnability is often thought to be part of usability, in fact, that is apparent in some usability evaluations and software quality standards such as the ISO/IEC 9126-1 and the IEEE Std. 610.12 [1]. However, in the revised version of the ISO standard for software quality, the ISO/IEC 25010 (that replaces the ISO/IEC 9126-1), learnability is included under the term flexibility [11].

As for the appearance of the various UI elements, the ones that have similar appearance should have similar functionality too. In other words, the similarity of the interfaces should guide the similarity of the operation or vice versa. This helps to cue the users with what to expect when performing an action on the UI. That in turns encourages us (i.e. the designers) to strive for achieving an external as well as internal consistency in the design ${ }^{3}$. An example for external consistency in NotesKB can be found in the Controllers Area of the Notepad View which follows the design of many navigation bars in other applications (e.g. Google Keep). When it comes to internal consistency, we tried to be consistent in using the same set of colors across the application. For instance, the blue color was persistently used to signify an element is selected or active.

\footnotetext{
${ }^{3}$ External consistency is a term used in HCI to refer to sharing the same design paradigm across different applications; while internal consistency applies to the usage of a given design paradigm in the same application. An example of an external consistency includes following the OS's particular navigation bar style (e.g. in Mac OS X versus in Windows) also the copy-paste keyboard shortcuts that work across applications. Internal consistency examples, on the other hand, include using the same style or set of symbols across the same application[80].
} 


\subsubsection{Direct Manipulation (DM)}

An interface style introduced by Shneiderman [98], the essence is to have objects of interest in a UI to represent actions visually and operate them directly without intermediaries. The main principles of the DM style include a) visual representation of "world of action"; b) fast, incremental and reversible actions; c) selection and pointing over typing; and d) immediate visual feedback.

Making an association between real-world objects and analogies is common in this design style so that they would help the user figure out how to operate the software. Analyzing the flexibility to initiate actions in this design style is important due to the differences in the users' backgrounds and abilities. In this style, elements of the interface are distinguished from each other depending on the type action, function or input they perform or handle [42]. These actions result in immediate visual feedbacks to the user, and they should achieve an acceptable degree of consistency in how they appear.

\subsubsection{Quiet Design}

As a concept, a quiet design emphasizes "less is more" [72]. Attention seeking should be purposeful, and for a clear reason, that would help the learner. Extra decorating, feature creep, and flashy interfaces are found to be disengaging to learners. Peters [72] mentions a study of a kindergarten classroom that illustrates how simplifying the learner environment contributes to more attention being paid in class, thus lead to better learning outcomes. The comparison was established by placing a group of children in minimally decorated classrooms and another in highly decorated ones, and the children in the former performed better concerning the amount of paid attention. That is not to say we should remove colors and artwork from learning interfaces, but a balance needs to be established to not distract the learner [72]. To illustrate the relevance of the quiet design in the context of learning environments, consider the study by Oviatt et al. [69]. The study involves an evaluation of the problem-solving skills of math problems done by high school students using different types of interfaces; 
namely pen and paper, digital stylus and paper, pen tablet and graphical tablet. The study concluded that many students felt that inputting math symbols using any method other than the simple pen and paper was harder to do and regarded the latter to be more preferable. In domains like mathematics, complex problem solving requires input fluency and flexible translation of different representations. Of course, that does not necessarily entail that using $\mathrm{PnP}$ is always viewed to be better; perhaps the mentioned MRAS [7] is a perfect contrary example to this one.

Thus, our striving for coming up with a quite design for the NotesKB's UI took a lot of effort and time. First, the selection of our UI color coding should look consistent and homogeneous throughout the interface. Also, they should serve a clear purpose, mostly for signifying something or some action in the UI. Finally, we attempt to make them in a way they appear noticeable enough but at the same time do not disturb the flow of the annotation task.

\subsubsection{Desirable Difficulty and Error}

On the visual part of the design, our job was not to make learning activities easy (e.g. math problems), but to make the interface "unthought of" [72]. That is achieved by simply eliminating unnecessary interface elements that slow down users, but not easing the learning task itself. That is to say, a desirable level of difficulty should exist in the design and be made on purpose to support the learning activity. Facing these obstacles in the interface will likely result in the users making errors. Error making is part of learning, and errors are categorized into three groups: a) positive consequences, b) negative consequences and c) neutral consequences. With positive consequences of errors, learners are more likely to continue achieving their learning goals after getting an undesirable result. With that in mind, we also tried to avert the expertise reversal effect; which states that what is true for a novice is not necessarily true for an expert user [72]. Therefore, interaction designers need to know their users first, and avoid applying any design strategies blindly. Many of the strategies listed in other publications will break down when it comes to experts. Most of the time, experts do not need many instructions on how to use a tool; instead, they need more 
control $[72,44]$.

\subsection{System Architecture and Implementation}

As mentioned earlier, NotesKB is a web-based tool where users can watch and annotate videos on YouTube; this section discusses the technologies and techniques used to achieve it. The tool was built as a client-server system; see the overall architecture of it (both the back-end and the front-end) and the interaction between them, in Figure 3.6.

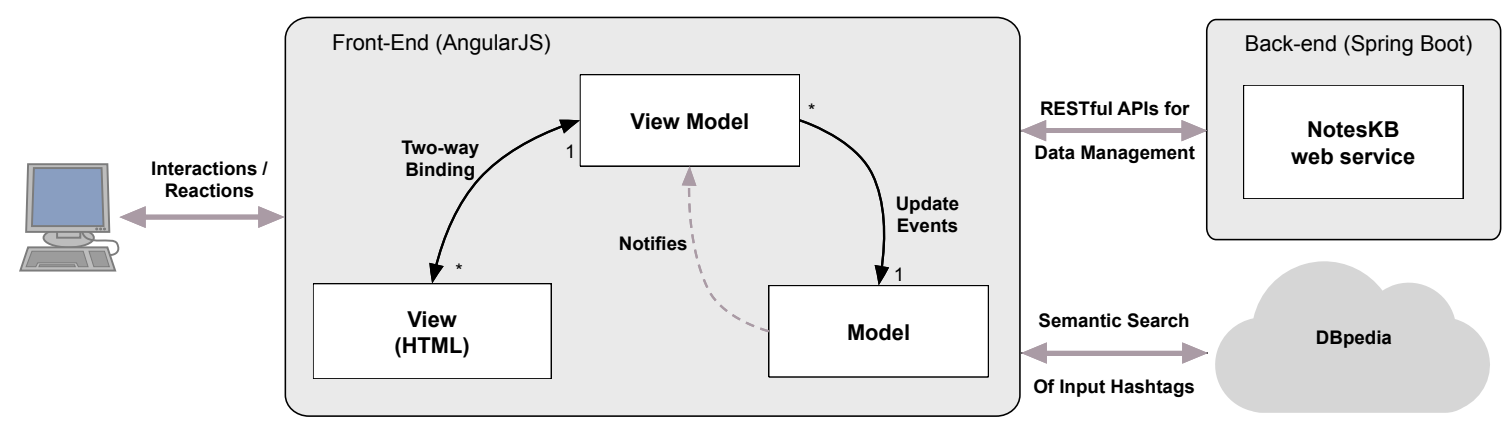

Figure 3.6: Simplified architecture of NotesKB video annotation system.

\subsubsection{Back-end architecture}

At the time when NotesKB was conceived, building an entire RESTful web service was not explicitly intended. REST stands for REpresentational State Transfer and involves queries specified in URIs [32]. However, over time and as the design evolved, it became apparent that building one was inevitable. Its (i.e. NotesKB server) job is to provide RESTful APIs for creating, managing and retrieving data and the NotesKB prototype web extension client integrates with users web browser and handles semantic video annotation authoring. As presented in Figure 3.7, there is the Archivable interface, and five fully implemented classes (Collection, Note, Annotation, Metadata, Hashtag) as well as the supporting AnnotationType enum class. The two main classes are the Collection class, and the Note class; and both implement the Archivable interface. The Note class has a dependency on the Hashtag class; an independent class is used 
to create and represent instances of written hashtags in the system. The remaining classes are merely compositions of the class Note.

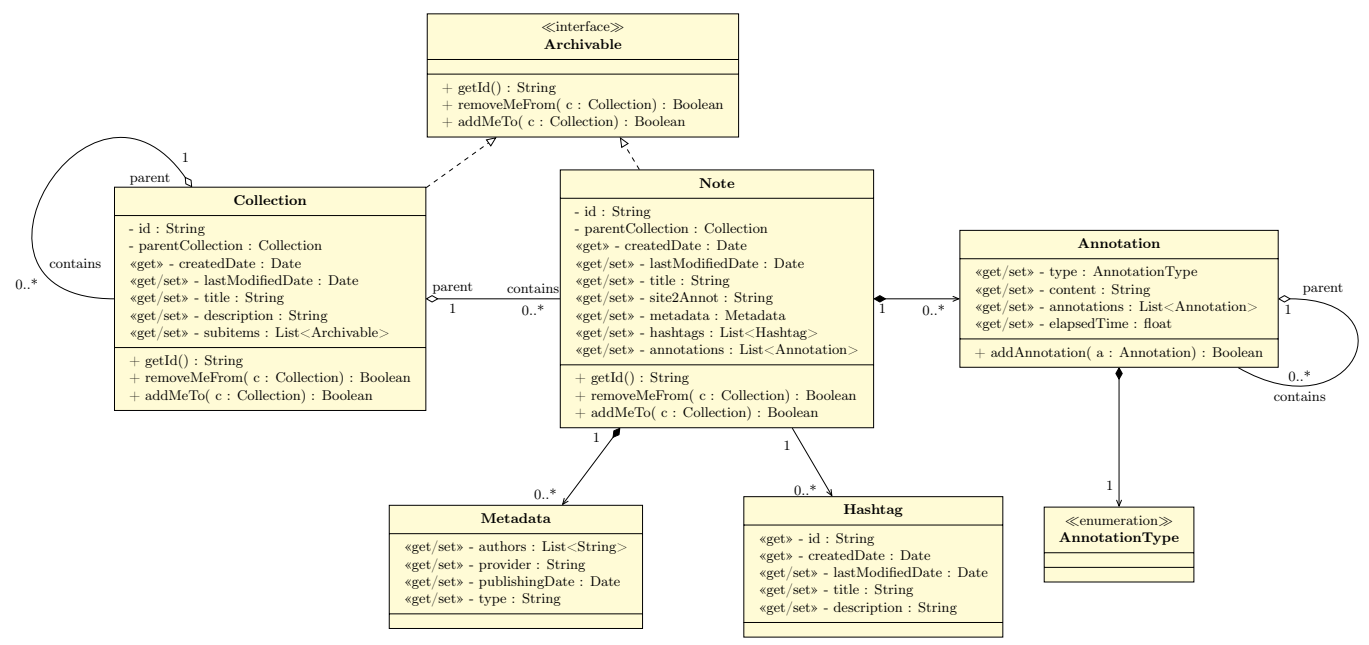

Figure 3.7: The class diagram of NotesKB's back-end.

When it came to building the service, the decision was made to develop the RESTful application using the Spring Boot framework on Java and rely on MongoDB for data persistence. The choice of the Spring Boot framework was made due to its attested ability to ease the developer's job of handling the various entities persistence inside the system, from building class schemas to making custom queries that manage information in the database with the minimum amount of code. Moreover, the framework has a good support for serving web applications built with HTML5 technologies. As we progressed through the development stages, we found out that the framework's highly accessible APIs to be quite reliable and rewarding in the long run.

Storing data in the system is a critical part of the service, and determining a candidate for that job has to go through a careful selection process. Persistence of our data has to be able to allow bidirectional relationships between different entities and at the same time it has to be scalable. First, we looked at using relational databases (e.g. MySQL, SQLite), but even though they are achievable to construct with all of the help that the Spring Boot framework provides, they pose a significant challenge nevertheless. The problem lies in system scalability. That became apparent to us as 
the number of our entities grew, and seemed that those relational databases would unlikely help us managing those entities thus hinder our efforts in the long run. To avoid such predicament, we looked at an alternative that is a non-SQL database, namely MongoDB. It was thought to be a viable solution that suits our needs quite well. The reasoning behind that comes from MongoDB's support of bidirectional relationships between classes within the Spring Boot framework as well as the ability to add on new properties to them in a reasonably straightforward way. This became evident as we noticed the same match between our model of the concrete classes and their counterparts in the database schemas model. Moreover, MongoDB keeps and stores data as JSON documents that can be relatively easy to retrieve from and export to other development environments.

\subsubsection{Front-end architecture}

This section mainly discusses the technologies used to develop the front-end of NotesKB, as well as some of the techniques they support. In conjunction with the typical usage of the primary Web technologies, namely HTML, CSS (Cascading Style Sheets) and JavaScript; AngularJS ${ }^{4}$ is the focal point that predominates our following discussions. AngularJS is an open-source framework built on top of JavaScript that provides the necessary APIs for building single-page applications for the Web, that is meant at saving the server-side (back-end) of the system the burden of providing clients with views each time a request is initiated. This is ideal since it keeps the responsibility of handling presentation logic to the client-side leaving the server-side a free hand to undertake business logic and storage. As a result, the Web application built using AngularJS become smoother, larger and more responsive.

The framework has gained a lot of popularity within Web development circles for its ability to tackle the problem of binding the system's data (model) to their HTML counterparts (view) in a seamless way. That is to say, when the model is modified, the HTML view that corresponds to it will be updated as a result and vice versa. From an architectural perspective, AngularJS makes a sensible use of the renowned

\footnotetext{
${ }^{4}$ https://angularjs.org/
} 
architectural pattern the Model-View-Controller (MVC) pattern. However, AngularJS goes a step further and accommodates another, related yet a slightly different pattern, known as the Model-View-ViewModel (MVVM) as well. In an attempt to avoid a terminologies application conflict, the AngularJS's principal developers team decided to coin the term Model-View-Whatever $\left(\mathrm{MV}^{*}\right)$ and provides a comfortable name that conforms to both patterns $[77,48]$. As for our implementation, we tend to depend on the MVVM more (please see Figure 3.6 for reference).
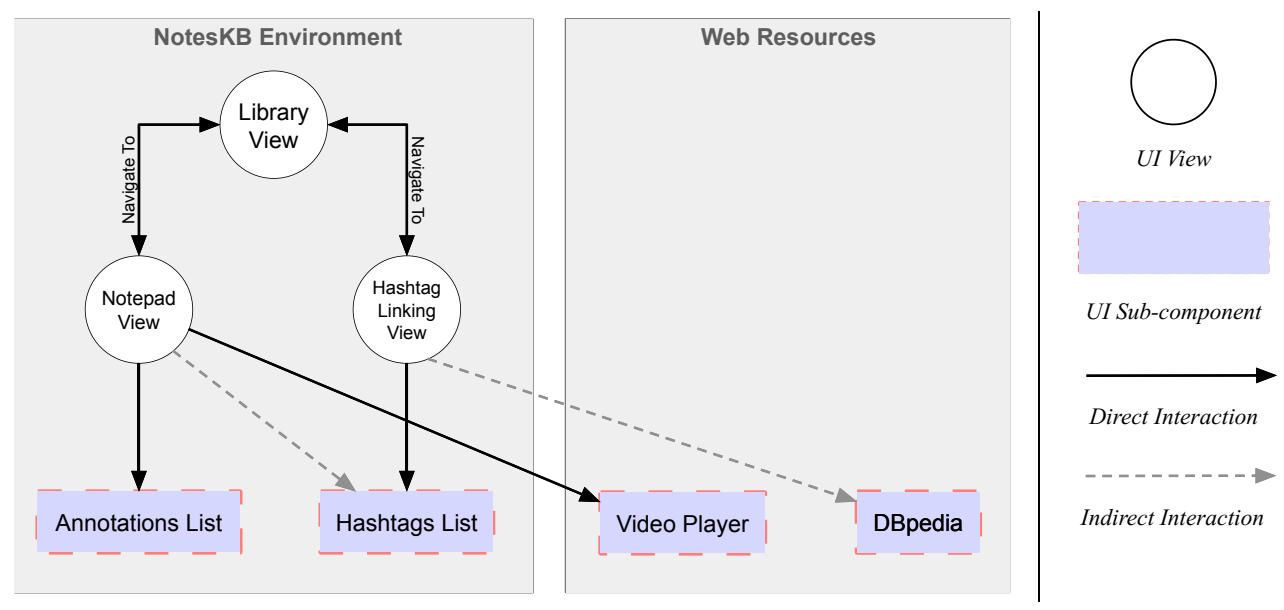

Figure 3.8: UI interaction flowchart diagram.

The front-end includes some pages, controllers, and other external dependencies. The pages, are what get represented to the user, and they are made of a mix of HTML tags with AngularJS elements. Each page is controlled by a controller. As explained earlier, they allow for our views (Library View, Notepad View, and Hashtag Linking View) to reflect data as they are modified. These views interact and are linked to each as in the illustration of Figure 3.8.

External resources such as videos and LODs are requested directly from their sources within the front-end framework. Videos are requested from YouTube along with their controllers; although we have augmented to them our own as in the example of the Controllers Area within the Notepad View (see Figure 3.4). For LODs, we depended on DBpedia to provide the system with them. Our requests are made exclusively 
to ontologies that are in the English language ${ }^{5}$. The following is a snippet of such requests made to the DBpedia service inquiring about "Khan_Academy":

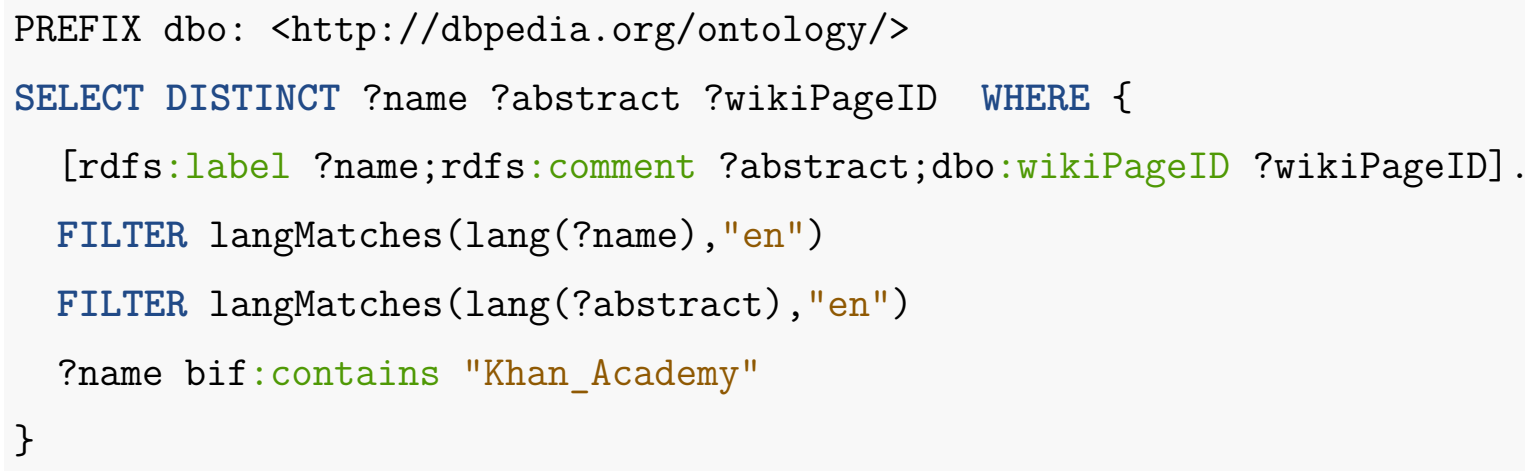

In such a query, we expect DBpedia to return a list of ontologies that are specified in English and that are of relation to the text "Khan_Academy." That connection between text and semantic is examined by conducting a basic text comparison of the string we provided with DBpedia's ontologies; specifically their "name" property. The "name" property can be thought of as a term that identifies the label a given ontology carries in a given language (English in our case). Once the query is complete, the list of ontologies that DBpedia provided will be displayed to the user to select from.

\footnotetext{
${ }^{5}$ Of course, this represents a limitation of the system, nevertheless we decided to go with it given that all of our tests and content we will test with will be communicated and conducted using English.
} 


\section{Chapter 4}

\section{Methodology}

Understanding the activity of annotating online educational videos, the main goal of the study, required from us conducting the evaluation in a lab setting. An examination period that was set between 90 - 120 minutes of running time, required recruiting participants that were university/college students only. These students (i.e. participants) were to provide us with the necessary data needed to complete evaluating the usability of the three different annotation methods (PnP, OneNote and NotesKB). Our study was granted a research ethics clearance from the Carleton University Research Ethics Board-B (CUREB-B), with Ethics Protocol Clearance ID: Project \# 106517.

Both quantitative and qualitative data of their experiences and performance were to be collected. Throughout this chapter, we will walk through the different data collection techniques and evaluation methods used to conduct this examination. To ensure the validity and reliability of our results, we have constantly sought using evaluation methods that were common and widely practiced in the field. Our test consists of four major parts (explained in details in Section 4.3); with each set to collect different information about the participants' experiences. At the heart of this examination, is the mentioned comparison between three different note-taking methods that are, the Pen and paper (PnP); the Microsoft OneNote; and our prototype NotesKB.

Our approach to testing these methods involved conducting an exploratory usability evaluations. As part of our evaluation, two factors were set to determine a students' performance in taking notes of online educational videos; the first one was the used annotation method and the other was the structure of the knowledge presented to them in the videos. Also, we depended on using some standardized usability questionnaires, namely SUS (System Usability Scale) and ASQ (After-Scenario Questionnaire). Among 
the advantages of using such standardized tests was to have measures that can be described as objective, replicable, quantifiable and scientifically generalized [83]. We had also relied on the comments of the participants describing their experiences throughout the testing sessions.

\subsection{Participants}

The targeted population for our study was university/college students. For practical reasons, our recruitment effort was exclusively targeted at recruiting students within Carleton University. The reason for choosing students was due to the presumption that students always take notes in their classes and are quite familiar with the note-taking process in general. Our sample includes 20 adult students aged between 18 and 35 years old, with 6 of them identified themselves as females and 14 as males. Most

of the participant (18 out of 20) were enrolled in a technology related field of study (e.g. computer science, HCI, engineering).

When it comes to the academic competence, participants varied in their level of studies they were pursuing at the time. The majority of them were highly educated; with two post-graduates and twelve graduate students. The rest were all undergraduate students. By observing their abilities in using the English language, the majority (17 out of 20) of them have demonstrated a high level to a moderate skill level linguistic abilities. Only three participants appeared to be struggling speaking or writing in English; as that can be observed from the vocal and written comments they left which we found it hard to understand and recognize the meaning behind them. Moreover, we also asked them about their academic performance. The majority of them rated their academic performance to be 'Above average student' (11 out of 20); while the rest were varied between either being an 'Average student' (4 out 20) or an 'Excellent student' (4 out 20). Only one participant declared himself to be a 'Struggling student.'

Lastly, the majority of participants stated that they did take notes on a regular basis for school (13 out of 20). Among the remaining participants who answered that they do not take notes on a regular basis, one mentioned that note-taking to him is 
dependent on whether or not the subject being studied is hard or not, and he would only take some notes when they are hard. All of the regular note-takers identified the Pen and paper (PnP) as their preferred medium to take notes for classes.

\subsection{Materials}

We ran our entire study, working both as a server and as a client, on an ASUS desktop PC (Intel® Core( $\left.{ }^{\mathrm{TM}}\right)$ i7-4770K CPU @ 3.50GHz, 4 Core(s), 16GB RAM, Microsoft Windows 10 Education OS). To monitor the participants' activities on the screen throughout our test, we relied on eye-tracking hardware and software by Gazepoint. The model of the eye-tracker we used in our study was called the Gazepoint GP3, and it was mounted on the 20 inch HP Pavilion 2010i Monitor display as illustrated in Figure 4.1. To record and analyze the screen-capture videos, the Gazepoint Analysis Standard Edition (v3.4.0) was used. It allowed recording activities of the participants on the computer screen along with the focus of their gazes. For creating and storing the answers of the participants to the pre-test and evaluation surveys, we used the LimeSurvey that was locally hosted in Carleton University's HotSoft lab.
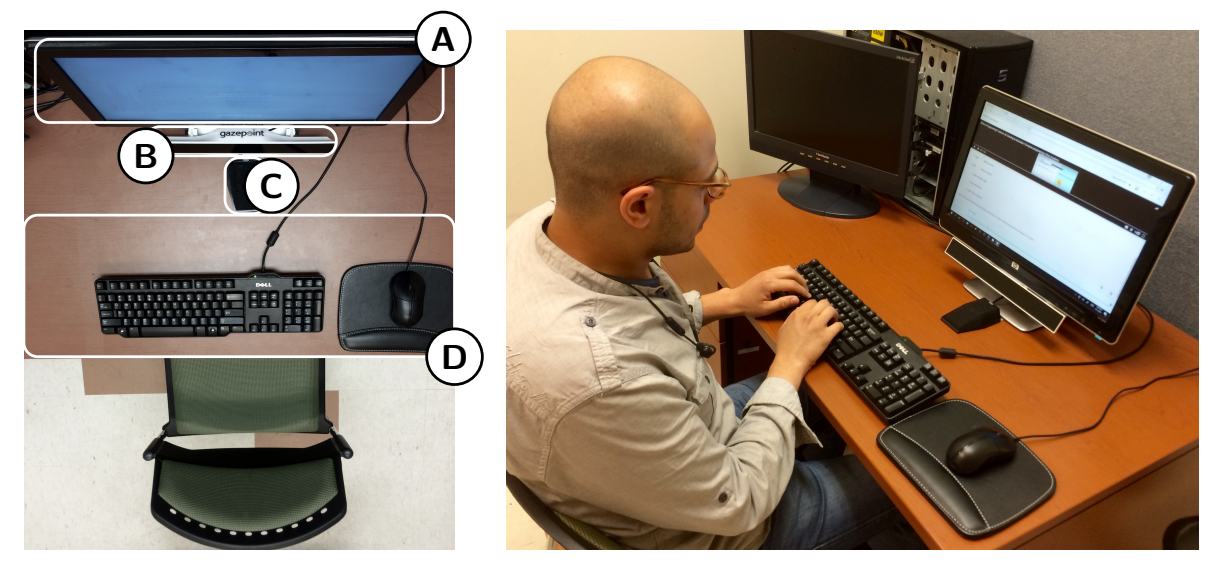

Figure 4.1: Overview of the testing setting - which includes (A) the main monitor display, (B) an eye-tracker, (C) a microphone, and (D) an input space [for mouse and keyboard also for PnP].

There were three videos selected and used as learning content, all of them were hosted 
on the YouTube website. The criteria for selecting a video was for it to be (a) explained in a clear English; (b) relatively short regarding duration and its running time fall in the range between 5 - 7 minutes; and (c) general (not specialized). The following lists the selected videos with a short description included (To read the videos' transcripts, see Appendix D):

- Video-A: titled as "Introduction to nouns," and it took 4 minutes and 27 seconds to finish and was made by Khan Academy [78]. In general, it can be described as a conceptual-based video that explains what constitutes a 'noun.'

- Video-B: this video was about the city of Ottawa's Tulip Festival and its origins. The video was titled "Did You Know? - History Of The Tulip Festival" and was made by the Cable Public Affairs Channel (CPAC) [20]. It ran for 5 minutes and 14 seconds and included so many historical events, thus can be described as a factual-based video.

- Video-C: this was another video made by Khan Academy, and it was titled "Firestick farming" [47]. The video discussed the use of fire by the indigenous people of Australia for farming, and it lasted for 6 minutes and 36 seconds. A mix of both conceptual, as well as factual information, was presented in the video.

As a side note, although we have collected eye-tracking data and even begun to analyze it, we eventually decided to abandon the idea because it was not necessary for this study's scope. One big predicament we faced in analyzing the eye-tracking data was the quality of the recorded gaze-traces. The quality was affected by a repeated sudden stops of the eye-tracker device. Add to that the variance occurred in finishing time and the screen size of the software tools during the experiment. The duration that took each participant to finish annotating a video varied from one participant to another; especially in Part 2. For example, "participant $\mathrm{X}$ finished annotating Video-B in 7 min while participant Y finished taking notes of it in 9.5 min"; that made analyzing eye-tracking videos even harder to deal with. Also, when analyzed the study, we found that some participants had re-sized the software windows to make it fit into their liking; which created an inconsistency in the collected visual data. 


\subsection{Procedure}

This section discusses in detail the four parts that comprised our test and what had happened in each one of them. That includes the given instructions and resources made available to each participant during the test. As Figure 4.2 shows, the four parts of our examination procedure were:

- Part1: Pre-test Questionnaires: To collect information about demographic, educational background as well as learning and note-taking habits.

- Part2: Note-taking Sessions: Three note-taking sessions, each followed by the post-study usability questionnaire SUS.

- Part3: UI Actions: Four UI actions performed using the NotesKB with each followed by the post-task usability questionnaire ASQ.

- Part4: User Interview: Three groups of open-ended questions aimed at finding out, from the participant's perspective, about their perceptions of the activities that took place in the previous parts of the test.

The participants arrived at the examination lab and were asked to sign a consent form affirming their participation in the study, if they agreed, they were briefly introduced to the research topic and made aware of its general objective. After checking the readiness of all equipment involved in the testing process, participants were instructed to go through each part, from Part 1 to Part 4 and think aloud as they were going through it. Towards the end of Part 4, participants were debriefed about the nature study they took part in and received a compensation of $\mathrm{C} \$ 20$ in cash.

\subsubsection{Part 1: Pre-test Questionnaires}

This is the first part of the testing procedure, in which the participant is simply asked to fill in two questionnaires using the LimeSurvey interface. The first questionnaire includes some demographic questions. It also asks some questions about the participant's general background; for example, usage of online social networks and consumption of Web contents. Following that was another questionnaire that was 
mainly focused on educational and learning activities practiced by the participant. For instance, participants were asked to rate their persistence in taking notes of classes on a regular basis and their preferred method to do so. (For reference, both questionnaires are included in Appendix B.)

\subsubsection{Part 2: Note-taking Sessions}

Here we describe Part 2 of the test, which included the three note-taking sessions. In each session, the participant was assigned an educational video to watch and take notes of using a particular note-taking method; starting with PnP, then OneNote and finally NotesKB. Given the variance in the videos' playing time, participants were allowed almost double that time to annotate the video's content. Thus, for Video-A and Video-B, participants were given 10 minutes to take notes of each one of them, and 15 minutes for Video-C. Further, those limits are applied to to avoid exhausting the participant and confine study's running time to be between 90-120 minutes.

Just before the first note-taking session (using PnP) started, a list of all of the commonly used annotation-types was handed to the participants that they could use as a reference (to review, please refer to Appendix E). For the following two sessions using OneNote and NotesKB, demo videos were created by us to show participants how to operate each software tool. The idea of showing these demos was to make sure that all participants have received the same standardized instructions as much as possible. This helped to reduce the possibility of that the researcher had forgotten to mention some information to the participant also keeping the examiner from interfering in the examination process to a bare minimum. However, the researcher was always available to answer any question the participant had after watching the demo. 


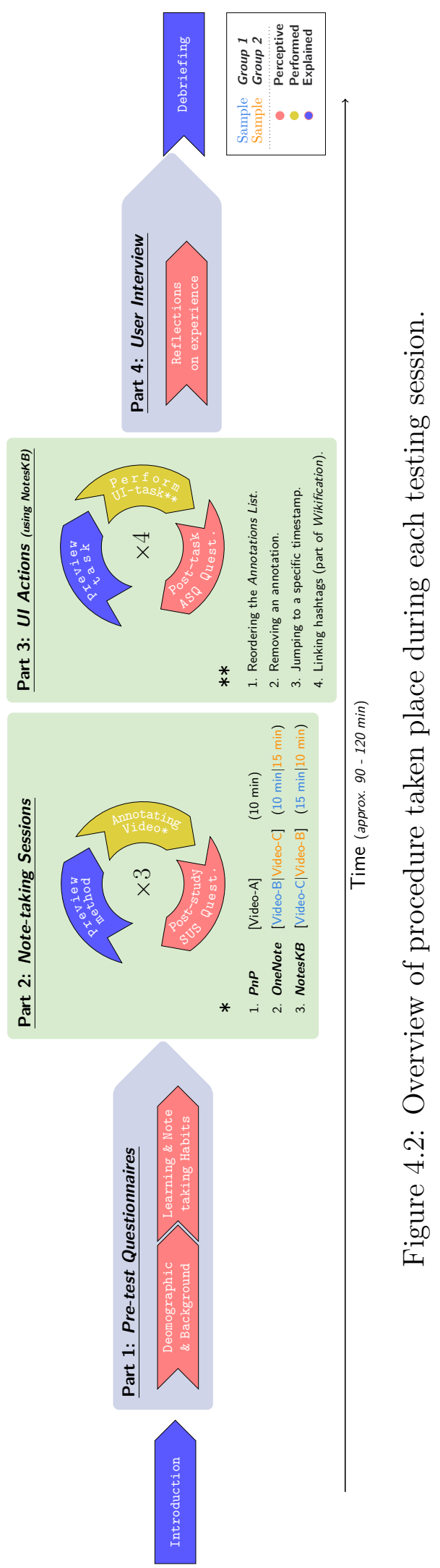


Participants were divided into two different groups, with each had the same number of participants (ten participants each). The first ten participants (P1-P10) were assigned to Group 1, and Group 2 had the rest of the participants, P11 through P20. The participants in both groups went through the same steps, except for the order they watched Video-B and Video-C. All participants started their note-taking sessions by annotating Video-A using PnP. After that, participants in Group 1 were instructed to use OneNote to annotate Video-B then move on to annotate Video-C using NotesKB. In Group 2, however, participants were instructed to annotate Video-C using OneNote, then annotate Video-B using NotesKB after (for visual clarification, please refer to Figure 4.2 and Table 4.2).

As already described, participants started the test by using PnP, then OneNote and finally NotesKB. The idea behind arranging the testing of methods in that order is to let the participant's transition from the most traditional way of note-taking ( $\mathrm{PnP}$ ) to the lesser one. Also, it was speculated that by following such order we would the participants get familiarized and lured into annotating the educational videos presented to them in the HiForm style. Part of emphasizing the use of this style is to encourage the inclusion of hashtags and annotation-types (Concept, Definition, Example) into notes.

Following each annotation session, participants were directed to fill in an SUS questionnaire evaluating the tool they just used and optionally add some written comments about it. SUS is post-study standardized usability test that consists of ten multiple choice questions, that each is evaluated using a 5-point scale. It is extensively used in industry and academia, and it is considered to be a valid and reliable measuring tool of usability of computer systems [82]. The SUS score first and foremost is concerned with measuring the usability of a system; however, upon wide examination of many SUS questionnaires, some argued that SUS encompasses learnability too [82, 54]. Two out of the questionnaire's ten questions (item 4 and 10) are used to evaluate learnability scale. While the remaining eight items are used to compute usability. As a questionnaire, SUS is limited in measuring the usability of a system as it only records the user satisfaction using that system leaving out things, such as effectiveness 
and efficiency out of that evaluation. Thus, one could argue it only measures the perception of usability [82].

The questions in the questionnaire alternate in tone back and forth from positive to negative. So, as its first question is worded positively (odd-numbered questions), the following question to it, is stated in a negative tone (even-numbered questions). That is one version of the SUS test, and it is known as the Standard version, and it is the version we used in this study. There is yet another equivalent version in which all questions are worded in a positive tone only, and it is known as the Positive version of SUS. There is no significant difference between results generated from both versions $[83,53]$ (to see the SUS questionnaire, please refer to Appendix C).

\subsubsection{Part 3: UI Actions}

This section is about Part 3 of the test, which is solely based on the participant performing some UI tasks using NotesKB. All tasks in this part were relatively brief; however, students could take as much time as they liked. The first three tasks were all performed within the Annotations List of the Notepad View (see Figure 3.4). Only in the fourth action, the participant was instructed to navigate to the Hashtag Linking View and use it (see Figure 3.5). The list includes all of the four actions in the order they are assigned accompanied by a short description:

1. Reordering the Annotations List: in this action, the participant is asked to re-position a particular annotation and be on the top of the Annotations List. Prior to performing this action, the participant is directed to access through the Library View an already made file note with the name 'What is Programming?' That same note file is used for action 2 and 3.

2. Removing an annotation: in this step, the participant is only asked to remove a particular annotation.

3. Jumping to a specific time-stamp: for this action, the participant is requested to play the part of the video in which the '\#angry_bird' is mentioned. Of course, the '\#angry_bird' is already present and included in the Annotations 
List; and all the participant has to do is to simply use the mouse to press on the hyper-linked time-stamp within the annotation (e.g. [0:59] jumps to the 59th second of the video's playing time).

4. Linking hashtags (part of Wikification): as mentioned previously in the previous chapter, Wikification is a process that consists of two steps; the first one is entering a hashtag followed by another in which user links that hashtag to a Wikipedia article that represents that hashtag. In this action, the participant is only doing the second step for three pre-determined unlinked hashtags ('\#khan_academy', '\#angry_birds' and '\#programming'). For each hashtag, the participant is tasked to search for a Wikipedia article that best corresponds to the hashtag being searched.

As the mentioned actions were being performed by the participants, the researcher was actively logging their progress in carrying out these actions and recording if they have completed them successfully or otherwise. For example, we maintained a record of each Wikipedia article of a given hashtag that was checked or linked by the participant. That, in turn, would help us determine if the participant had linked the correct article to the hashtag or not as we will see in the next Results chapter. Subsequently, after executing each action, they were asked to fill up an ASQ questionnaire (to see the ASQ questionnaire, please refer to Appendix C).

ASQ is a post-task standard usability questionnaire that consists of 3 questions. The answer to each question is represented on a scale from 1 to 7 , with score 1 representing the most satisfying experience and 7 is its least satisfying to the user. The three evaluation questions of ASQ measure the degree in which the participant was satisfied carrying out the task in terms of its easiness, the time it consumed to finish as well as the supporting information provided with it. Once the scores of these questions are recorded, an overall average score is then calculated per participant. Unlike SUS, the ASQ is designed to assess the participant's satisfaction doing a specific task rather than a whole UI study. However, just like the SUS, ASQ produces reliable results as well [83]. 


\subsubsection{Part 4: Interview}

Here we describe the last part of the test in which the researcher asked the participant questions, mostly open-ended ones, about his/her experience. The questions revolve around three themes (to preview a sample of a conducted interview, please refer to Appendix F):

1. Most/Least preferred annotation method to use. By asking the participant about his/her most and least preferred note-taking method, we aim at gaining insights of whether or not our collected scores from the SUS questionnaires match those of students' perceptions about the three methods.

2. Participant's opinion about the general practice of annotating online educational videos.

3. Participant's opinion about the intrinsic complexity of the watched videos. So, we asked participants a direct question about which video they thought was the longest in duration. Although Video- $\mathrm{C}$ was the longest, we were interested in asking participants this question to detect if the density of the learning content presented in Video-B had led some students to think it was longer.

\subsection{Analysis Plan}

This section summarizes the independent variables (i.e., factors) dependent variables (i.e., measures) used in the testing. As mentioned in the previous section, participants were divided into two groups (Group 1 and Group 2). Following a within-subject design, all participants experienced the same tasks except for the exposure order of the videos noted earlier. Setting up the evaluation that way was thought to help us maximize the amount of data we can collect from participants during the testing sessions.

To recap, investigating the activity of note-taking of online educational videos is our main objective in this study, and the goal is to provide some answers to the following 
research questions:

- What is the usability of using PnP, OneNote, and NotesKB to annotate online educational videos?

- What are the factors that affect the note-taking experience of online educational videos the most?

- Does the annotating style affect the annotation outcome?

Thus, in a way, the design of our study had to address each of these questions and collect as much data to help answer them. In the upcoming subsections, we define the different variables we used to conduct the study and the rationale behind choosing them.

\subsubsection{Independent Variables (Factors)}

There are two major factors that we controlled during the examination process. The first factor was the tools used to annotate the videos, while the second one was the videos being annotated. Although the nature of educational contents presented in videos was quite limiting in the fact that they were mainly focused on factual and conceptual types of knowledge; we think they still reveal some unknown aspects about the note-taking experience and show us the different dynamics involved when using different annotation methods.

- Annotation method: represent the method or tool used to take down notes of the educational video.

- Video's knowledge structure: this variable is related to the nature of the educational materials presented to the students in the videos. Based on the Structure of the Knowledge Dimension of the Bloom's Taxonomy, we found that the selected videos can fall into one its categories. So, Video-A mostly includes conceptual knowledge, and Video-B is mostly factual knowledge and Video-C provides a mix of the both (conceptual and factual). Even though this categorization can be said to be arbitrary, yet the fact remains that the knowledge within them is hard to be described as procedural nor as meta-cognitive knowledge. 
Table 4.1: Table of the independent variables along with their levels.

\begin{tabular}{cc}
\hline Independent Variables (Factors) & Levels \\
\hline Video's knowledge structure & Conceptual based, Factual based, Mixed \\
Annotation method & PnP, OneNote, NotesKB \\
\hline
\end{tabular}

Table 4.2: Table with the study trials ordered as they were experienced by both participant groups.

\begin{tabular}{ccccc}
\hline Order & $\begin{array}{c}\text { Annotation } \\
\text { Method }\end{array}$ & $\begin{array}{c}\text { Conceptual Based } \\
\text { (Video-A) }\end{array}$ & $\begin{array}{c}\text { Factual Based } \\
\text { (Video-B) }\end{array}$ & $\begin{array}{c}\text { Mixed } \\
\text { (Video-C) }\end{array}$ \\
\hline 1 & PnP & $\sqrt{ }(\mathrm{G} 1, \mathrm{G} 2)$ & - & - \\
2 & OneNote & - & $\sqrt{ }(\mathrm{G} 1)$ & $\sqrt{ }(\mathrm{G} 2)$ \\
3 & NotesKB & - & $\sqrt{ }(\mathrm{G} 2)$ & $\sqrt{ }(\mathrm{G} 1)$ \\
\hline
\end{tabular}

\subsubsection{Dependent Variables (Measures)}

These variables are aimed at measuring human behavior. However, when it comes to annotating educational contents of any kind (online educational videos included), then the question becomes what measures we can use to quantify such activity; especially when the study is not concerned at measuring learning outcomes? We used the following measurements:

- Usability (SUS): as stated earlier, SUS is a post-study questionnaire designed to measure how usable a system is. We used this as a measure to compare the three different annotation methods.

- Satisfaction (ASQ): unlike SUS, this post-action questionnaire is designed to determine the satisfaction level of a user carrying out a specific action in the UI. We used this measure for Part 3, which involved using NotesKB's UI exclusively.

- Perceived complexity: this looks at whether or not the participant thinks that the intrinsic complexity of the educational videos shown had an affect on 
his/her annotation experience. So, in the interview, participants were asked about whether or not the presented educational material had affected his/her own experience in any way. They were also asked about which video they thought to be the longest regarding duration.

- Quality of Wikification Linkage: an average score of the number of times the participant had successfully selected the correct Wikipedia article that corresponds to a given hashtag. 


\section{Chapter 5}

\section{Results and Discussion}

The results obtained from the various tests we conducted are presented and discussed in this chapter. Given the dissimilarities of collected data from the study, we tried our best to address them in graphs and tables that are easy to read and convey our points. The section is broken down into two major sections; Results (Section 5.1) and Interpretation and Discussion (Section 5.2). Throughout the subsections of each, we will tackle the findings we have obtained from conducting this examination.

When participants were interviewed about their preferred annotation method they have just experienced, NotesKB was selected by half of them (see Table 5.1).

\section{$5.1 \quad$ Results}

As both qualitative and quantitative data were obtained at the same stage of the study, this section lays out the general outcomes from the study. For the qualitative data, we applied the thematic analysis method on collected data, chiefly based on the participants' comments and answers to the interview open ended questions [21]. We first reviewed all of the transcripts and notes and applied open coding, and then identified the key themes and how they relate to each other. For the quantitative data, however, we depended on the formal recorded data such as the standardized tests and other survey results. For simplicity, processed data is described sometimes in tables and other graphical data representations such as histograms to convey some statistical information about it. Completed statistics are included in subsections 5.1.1 or 5.1.2; while the thematic analysis is part of subsection 5.1.3. 
Table 5.1: Summaries of the answers during the interviews with participants.

\begin{tabular}{lcc}
\hline Method & Most preferred & Least preferred \\
\hline PnP & 8 & 4 \\
OneNote & 2 & 11 \\
NotesKB & 10 & 5 \\
\hline
\end{tabular}

(a) Annotation method preference.

\begin{tabular}{lc}
\hline Video & Freq $^{*}$ \\
\hline Video-A & 0 \\
Video-B & 7 \\
Video-C & 11
\end{tabular}

(b) Longest perceived video.

\begin{tabular}{llcc}
\hline & N/A & No & Yes \\
\hline Found annotating videos appealing & 2 & 4 & 14 \\
Learning material affected performance & 1 & 8 & 11 \\
\hline
\end{tabular}

(c) Yes/No questions.

* Some participants did not answer the questions.

\subsubsection{Descriptive Statistics}

Statistics shown in this part are used to arrive at measurable outcomes to provide estimates of data collected. Outlined in the following subsections, we broke down our statistics into two groups, one that is concerned with the data collected from the surveys before the evaluation process took place, followed by another that describe data gathered later.

\subsubsection{Pre-Test Data}

Given that all of our participants were university students, describing the different demographics and backgrounds they belong to is thought to be of importance to understanding the study. As we can observe from Figure 5.1 and Figure 5.2, to some extent, the demographics of our participants are skewed. At least half of the participants were male graduate right-handed students enrolled in a technology influenced academic program (mostly Computer Science) with an above average academic performance and aged between 20-30 years old. That description may not be that critical in explaining the results of our study, but it could shed some light 
on the efficacy of the annotation methods tried out and how far we could generalize them.
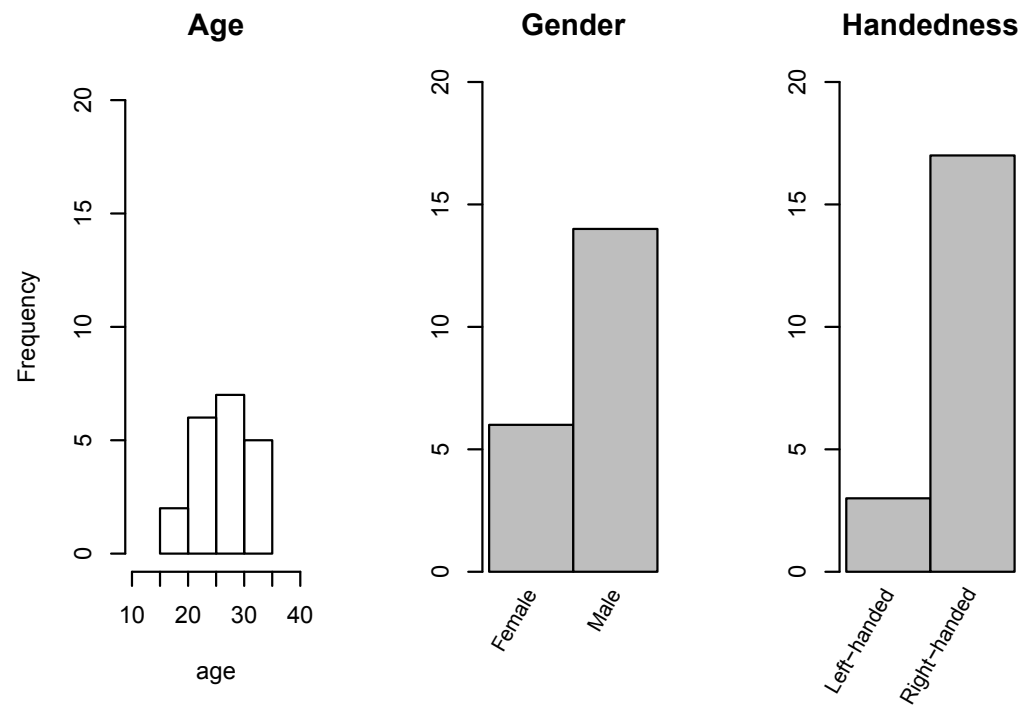

Figure 5.1: Graphical representations of demographic data.

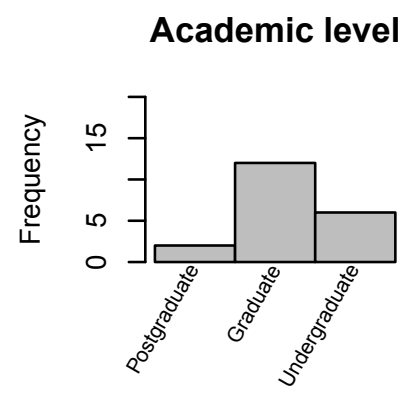

Academic performance

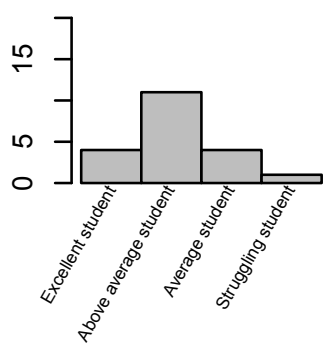

Academic programs

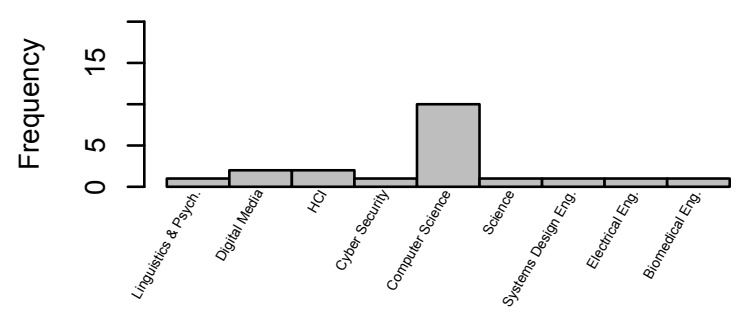

English language level

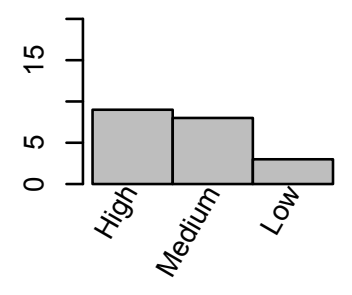

Figure 5.2: Graphical representations of data of the students' academic backgrounds and general skills.

Commonly used social network

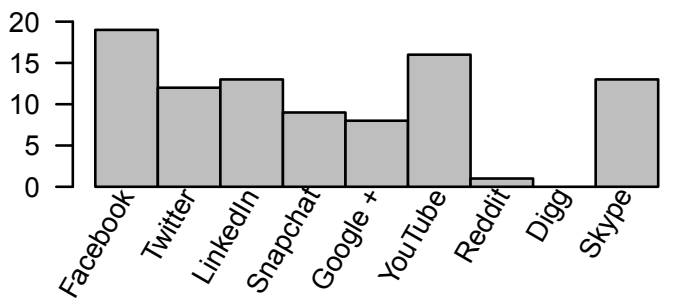


When it comes to the Web content consumption habits, as expected of such targeted population, all of the students who participated in the study seemed to be coping well with what contemporary cyberspace has to offer and its various technological trends (see Figure 5.3). For the majority of the participants, it seems apparent that they consume much content online on a daily basis; for entertainment, socializing (on Facebook especially), as well as for work and education (see Figure 5.4). Thus, one can disregard that the testing sample was unequipped or out of touch using current technologies. Facebook, YouTube, and LinkedIn come on top as the most common social media used by the participants (see Figure 5.2). Several of the networks do support the use of hashtags, or a mechanism similar to it at least. This suggests that the idea of using hashtags is quite unlikely to be alien to these participants.

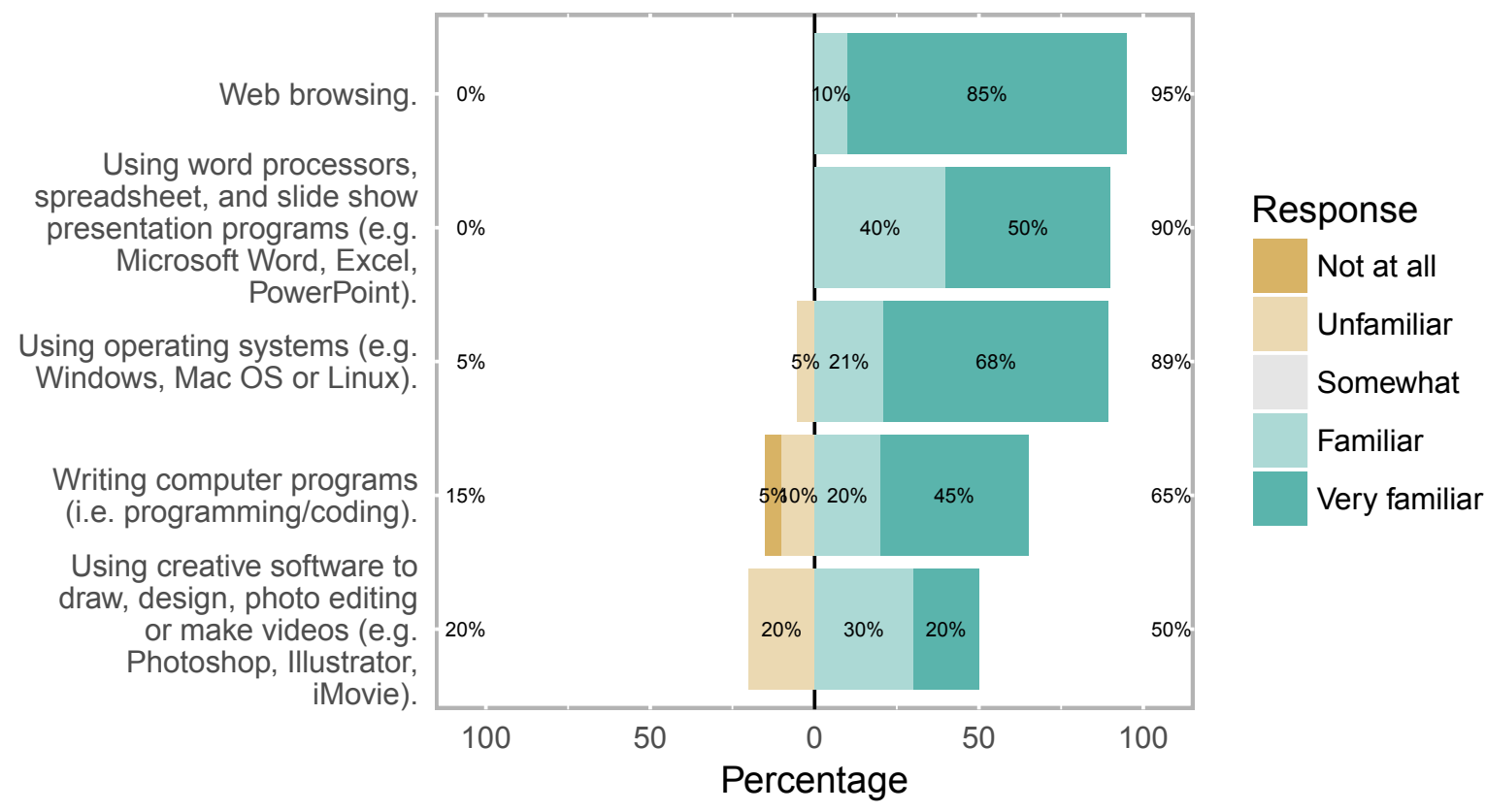

Figure 5.3: Participants' ratings of their computer skills.

When it comes to the statistics about the participants' note-taking habits, however, we see a certain degree of divergence between participants. In this, participants' responses seem to be quite diverse when it comes to the note-taking abilities and habits. As we can see from Figure 5.5, although the majority of the students had specified that they are capable of summarizing their notes using their own words, they diverged in the degree of their capabilities. Another element where the participants' responses varied 
widely in their commitment to the practice of editing personal notes within 24 hours after class.

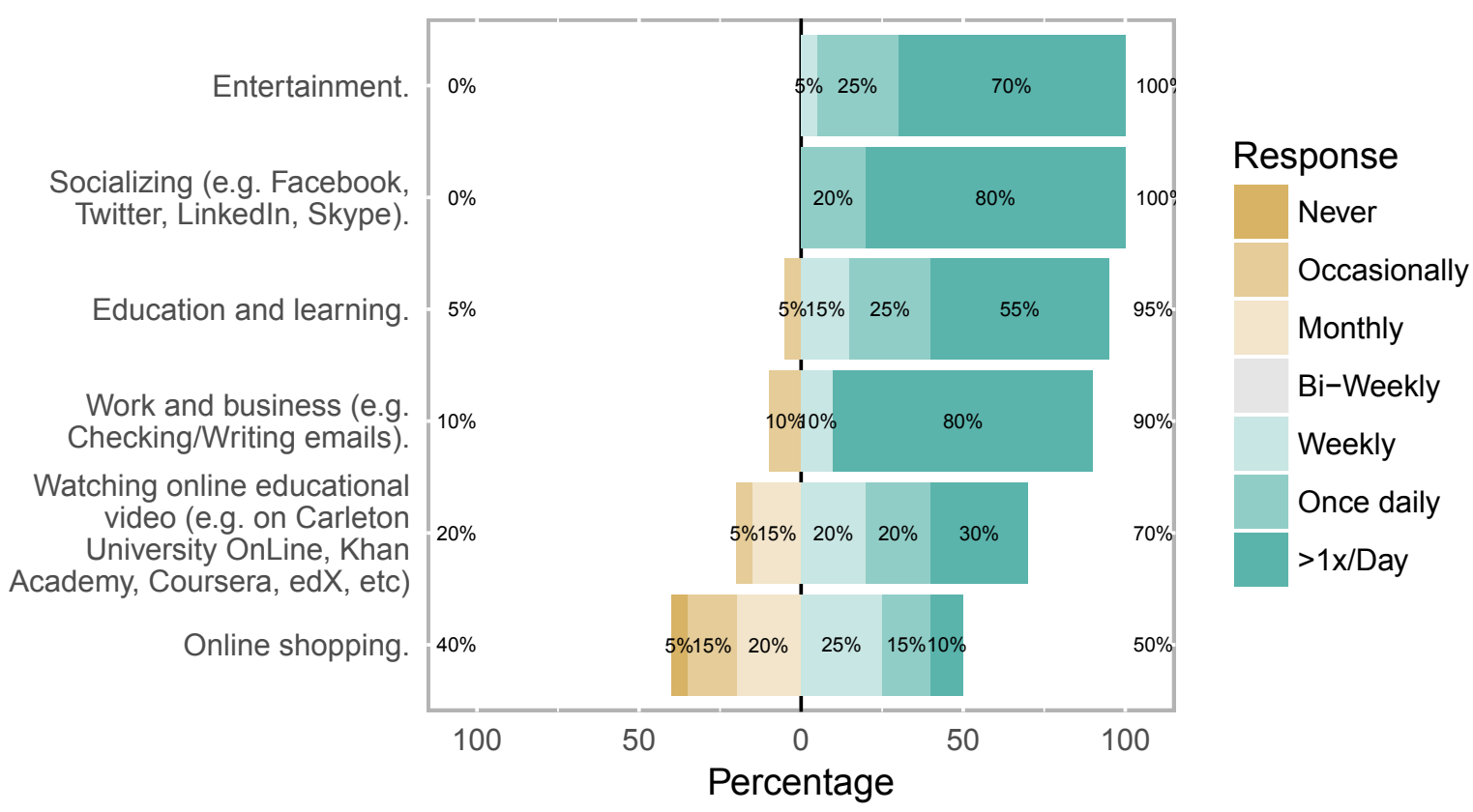

Figure 5.4: Usage patterns of different activities on the Web.

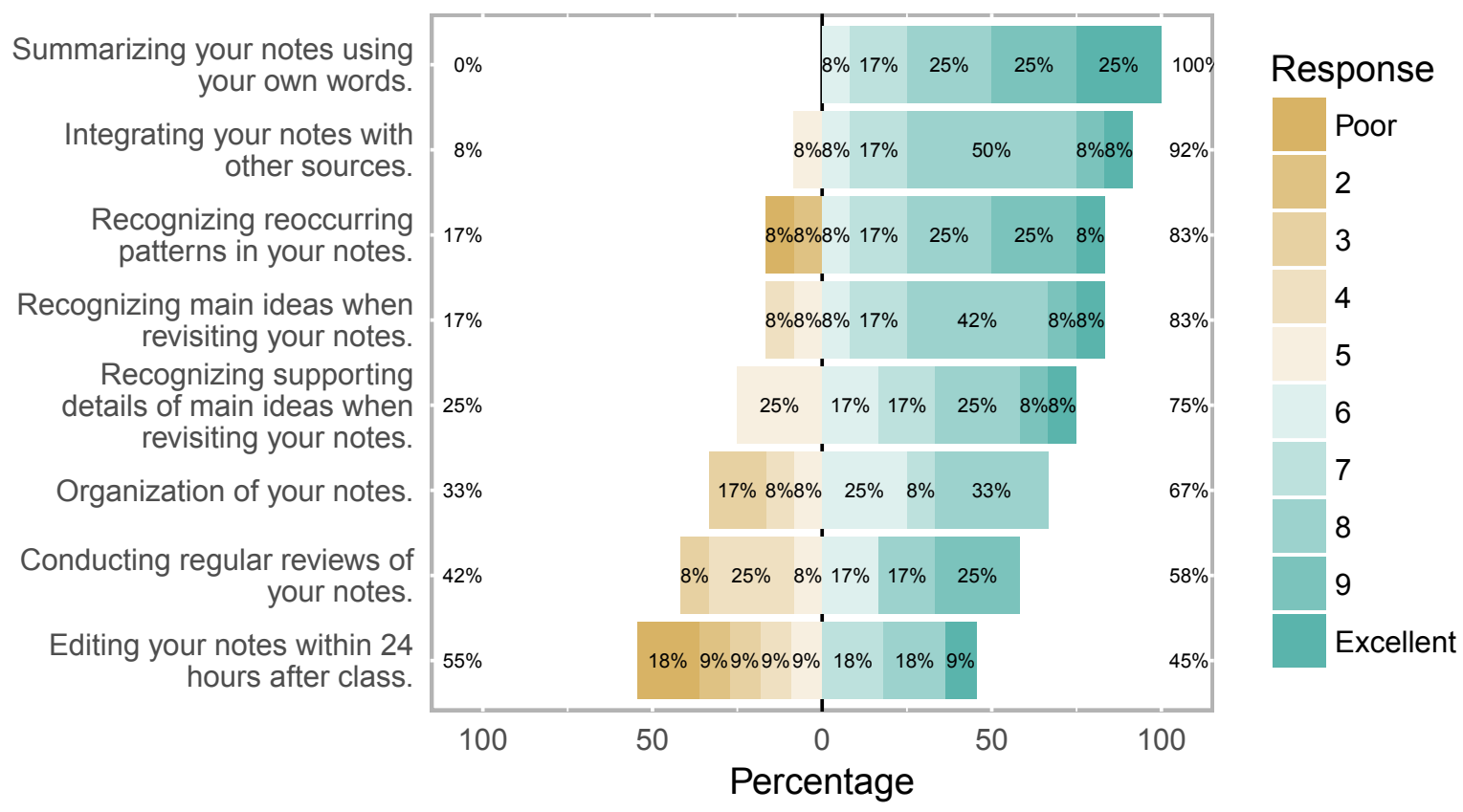

Figure 5.5: Participants' ratings of their note-taking skills and habits. 


\subsubsection{Standardized Tests}

\subsection{SUS Scores}

As Table 5.2 shows $^{1}$, PnP earned the highest SUS average score of 80.9 followed by NotesKB (avg. 62.2) and finally OneNote (avg. 55.9). As it turns out, there is no significant difference between the NotesKB and OneNote when it comes to the overall SUS score. Also, as Figure 5.6 illustrates, the differences between the two participants groups, G1 and G2, are thought to be arbitrary and are little or no significance.

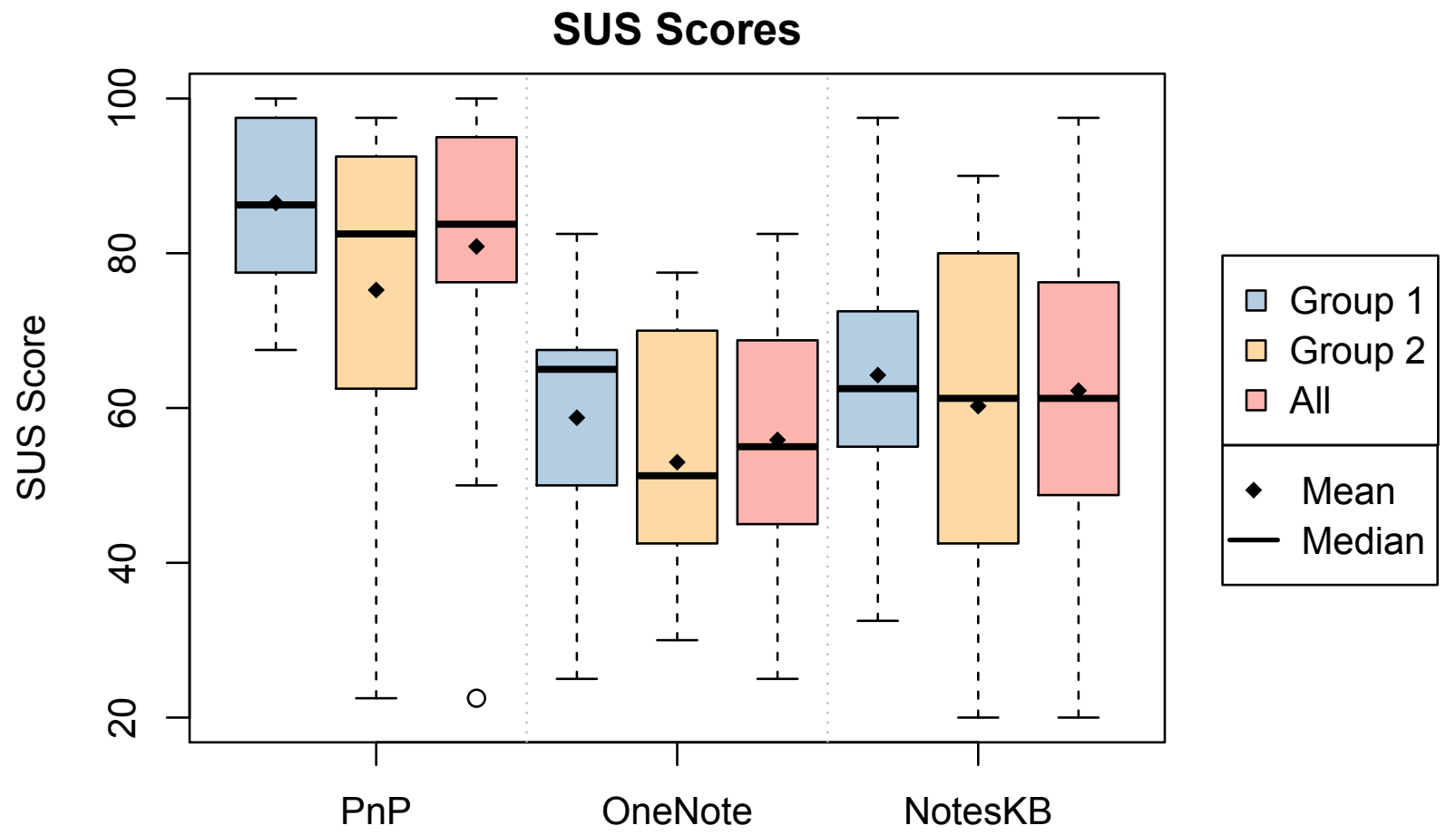

Figure 5.6: Boxplots displaying the distribution of collected SUS scores.

Apart from the overall SUS scores, a notable difference exists in the 'learnability' score of all three methods. PnP has the highest average score, again, with OneNote scoring a higher average (73.1) than that of NotesKB (60.6). We found the close

\footnotetext{
${ }^{1}$ Please note that the numbers presented here in this section are not percentile ranks, they are mere calculations applied to the collected raw SUS scores as they were recorded (please refer to Sauro [82] and Sauro and Lewis [83] for more reading about making the conversion from raw SUS scores to percentile ranks).
} 
approximation between the different medians and means across all methods to be another interesting distinction found in the results. This close approximation somewhat positively indicates that the collected data is distributed evenly over the total range (see Figure 5.6 and Table 5.2 for reference).

\subsection{ASQ Scores}

The ASQ score records the level of satisfaction participants felt after completing a test scenario. In Figure 5.7, one can easily spot the variance between the ASQ scores of each task. Most participants were overwhelmingly satisfied when they completed Action 2 and Action 3. On the other hand, the level of satisfaction executing Action 1 and Action 4 ranges widely from unsatisfying to satisfying; though it leans more toward the side of the latter. Nevertheless, we think it is normal for both actions (i.e., Action 1 and Action 4) to be viewed that way given that they do require more steps to carry out than the other two. (Action 1 involved finding buttons in the UI while Action 4 is a hashtag linking task.)

\section{Unsatisfied ASQ Scores}

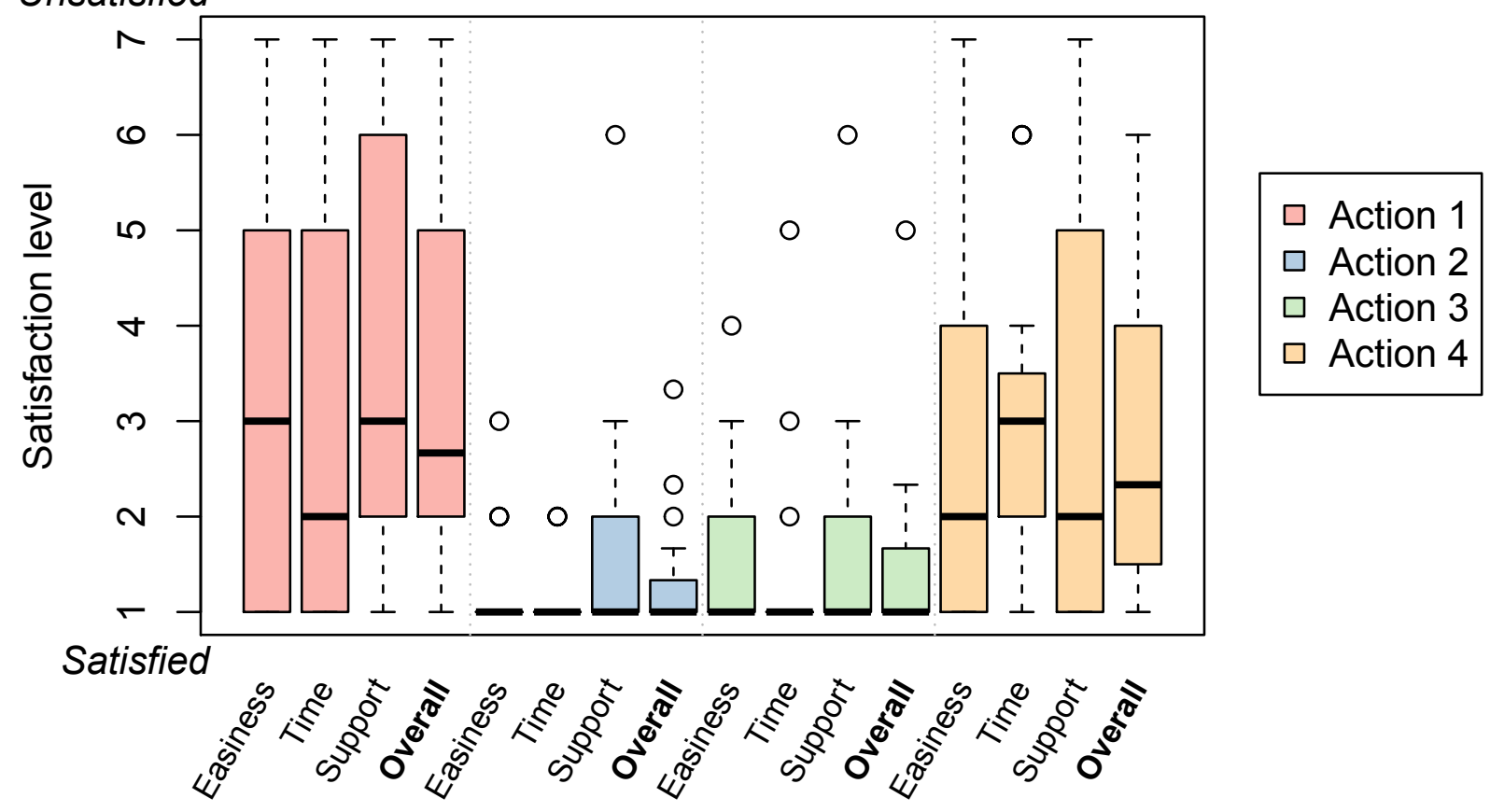

Figure 5.7: Boxplots displaying the distribution of collected ASQ scores. 
Table 5.2: SUS results.

\begin{tabular}{|c|c|c|c|c|c|c|c|c|c|}
\hline \multirow[b]{3}{*}{ Measures } & \multicolumn{9}{|c|}{ Annotation Method } \\
\hline & \multicolumn{3}{|c|}{$P n P$} & \multicolumn{3}{|c|}{ OneNote } & \multicolumn{3}{|c|}{ NotesKB } \\
\hline & G1 & G2 & All & G1 & G2 & All & G1 & G2 & All \\
\hline \multicolumn{10}{|l|}{ SUS Score } \\
\hline Mean & 86.5 & 75.2 & 80.9 & 58.8 & 53.0 & 55.9 & 64.2 & 60.2 & 62.2 \\
\hline Median & 86.2 & 82.5 & 83.8 & 65.0 & 51.2 & 55.0 & 62.5 & 61.2 & 61.2 \\
\hline Std Dev & 11.6 & 23.7 & 19.0 & 16.4 & 17.1 & 16.6 & 19.7 & 22.4 & 20.6 \\
\hline Std Err & 3.7 & 7.5 & 4.3 & 5.2 & 5.4 & 3.7 & 6.2 & 7.1 & 4.6 \\
\hline Low $*$ & 78.2 & 58.3 & 72.0 & 47.0 & 40.8 & 48.1 & 50.2 & 44.2 & 52.6 \\
\hline High * & 94.8 & 92.2 & 89.8 & 70.5 & 65.2 & 63.6 & 78.3 & 76.3 & 71.9 \\
\hline Min & 67.5 & 22.5 & 22.5 & 25.0 & 30.0 & 25.0 & 32.5 & 20.0 & 20.0 \\
\hline $\operatorname{Max}$ & 100.0 & 97.5 & 100.0 & 82.5 & 77.5 & 82.5 & 97.5 & 90.0 & 97.5 \\
\hline \multicolumn{10}{|l|}{ Usability } \\
\hline Mean & 84.4 & 73.8 & 79.1 & 53.4 & 49.7 & 51.6 & 64.1 & 61.2 & 62.7 \\
\hline Median & 84.4 & 81.2 & 81.2 & 57.8 & 46.9 & 54.7 & 60.9 & 65.6 & 64.1 \\
\hline Std Dev & 13.5 & 23.5 & 19.5 & 17.6 & 17.8 & 17.3 & 18.1 & 21.2 & 19.2 \\
\hline Std Err & 4.3 & 7.4 & 4.3 & 5.6 & 5.6 & 3.9 & 5.7 & 6.7 & 4.3 \\
\hline Low $*$ & 74.7 & 56.9 & 70.0 & 40.8 & 37.0 & 43.4 & 51.1 & 46.1 & 53.6 \\
\hline High $*$ & 94.0 & 90.6 & 88.2 & 66.1 & 62.4 & 59.7 & 77.0 & 76.4 & 71.7 \\
\hline Min & 59.4 & 25.0 & 25.0 & 15.6 & 25.0 & 15.6 & 37.5 & 18.8 & 18.8 \\
\hline Max & 100.0 & 96.9 & 100.0 & 78.1 & 78.1 & 78.1 & 96.9 & 87.5 & 96.9 \\
\hline \multicolumn{10}{|l|}{ Learnability } \\
\hline Y & $95 .($ & 81.2 & 88.1 & 80.0 & 66.2 & 73.1 & 65.0 & 56.2 & 60.6 \\
\hline Median & 100.0 & 93.8 & 100.0 & 81.2 & 62.5 & 68.8 & 62.5 & 56.2 & 62.5 \\
\hline Std Dev & 8.7 & 27.8 & 21.3 & 17.9 & 25.0 & 22.3 & 29.3 & 31.9 & 30.2 \\
\hline Std Err & 2.8 & 8.8 & 4.8 & 5.7 & 7.9 & 5.0 & 9.3 & 10.1 & 6.7 \\
\hline Low $*$ & 88.7 & 61.4 & 78.2 & 67.2 & 48.3 & 62.7 & 44.0 & 33.5 & 46.5 \\
\hline High $*$ & 101.3 & 101.1 & 98.1 & 92.8 & 84.2 & 83.6 & 86.0 & 79.0 & 74.7 \\
\hline Min & 75.0 & 12.5 & 12.5 & 50.0 & 37.5 & 37.5 & 12.5 & 12.5 & 12.5 \\
\hline Max & 100.0 & 100.0 & 100.0 & 100.0 & 100.0 & 100.0 & 100.0 & 100.0 & 100.0 \\
\hline
\end{tabular}

\footnotetext{
'Low' and 'High' refer to the Confidence interval
} 


\subsubsection{Comparisons}

Drawing upon information obtained from the previous subsection 5.1.1, in this part, we will attempt to discover relationships between various results using inferential statistical methods and techniques to reach such conclusions that eventually support our hypothesizes.

To detect differences between the SUS scores of the three annotation methods, we relied on the non-parametric Kruskal-Wallis analysis. From applying the analysis on our data sets, we get the $p$-value $<0.001$; which indicates an existence of a significant difference between the three note-taking methods without specifying which one. To confirm where the difference was taking place, we tested the three methods in pairs, and compare each pair by applying the Wilcoxon signed rank test with continuity correction. As it turns out, results suggest that $\mathrm{PnP}$ is the method that is showing a significant difference when compared to the other two methods. For OneNote and NotesKB, however, results show no significant difference between the two. To correct the $p$-values, we multiplied them by three given that we made three comparisons (see Table 5.4).

Another important variance noticed relates to Action 4. We learned from the ASQ score (Section 5.1.1.2.2) that participants had different views about each of the four actions. However, that variation was based on the participant's point of view and their performances. Here we will dive deeper into Action 4 and investigate the completion rate in finishing the Wikification process. The most important indicator we are interested in is the rate of how many participants had successfully linked the Wikipedia articles to the designated hashtags. Whether the participants had opened the actual Wikipedia article and read them or if they had edited a given hashtag and make it comply with the name of the intended article is thought to be of secondary importance. As the results in Figure 5.8 illustrate, it seems that linking the right Wikipedia articles to the hashtags is content dependent. That is to say, the "\#khan_academy" hashtag was linked completely by most of the participants, and we

suspect the reason for that is due to the fact that the Wikipedia article correspond to 
Table 5.3: ASQ results.

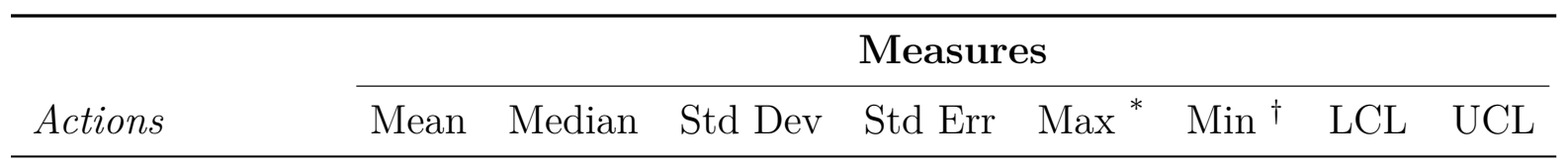

Action 1: Reordering the Annotations List

$\begin{array}{lcccccccc}\text { Easy to do } & 3.29 & 3 & 2.28 & 0.52 & 7 & 1 & -3.28 & 9.87 \\ \text { Time avaliable } & 3.11 & 2 & 2.27 & 0.52 & 7 & 1 & -2.5 & 8.73 \\ \text { Info support } & 3.71 & 3 & 2.11 & 0.49 & 7 & 1 & -1.77 & 9.18 \\ \text { Overall } \ddagger & 3.43 & 2.67 & 2.01 & 0.46 & 7 & 1 & -1.84 & 8.7\end{array}$

Action 2: Removing an annotation

$\begin{array}{lllllllll}\text { Easy to do } & 1.21 & 1 & 0.54 & 0.12 & 3 & 1 & 0.37 & 2.05 \\ \text { Time avaliable } & 1.11 & 1 & 0.32 & 0.07 & 2 & 1 & 0.83 & 1.39 \\ \text { Info support } & 1.67 & 1 & 1.28 & 0.29 & 6 & 1 & -0.99 & 4.33 \\ \text { Overall } & 1.32 & 1 & 0.62 & 0.14 & 3.33 & 1 & 0.1 & 2.53\end{array}$

Action 3: Jumping to a specific timestamp

$\begin{array}{llllcllll}\text { Easy to do } & 1.53 & 1 & 0.9 & 0.21 & 4 & 1 & -0.43 & 3.49 \\ \text { Time avaliable } & 1.37 & 1 & 1.01 & 0.23 & 5 & 1 & -0.31 & 3.05 \\ \text { Info support } & 1.61 & 1 & 1.29 & 0.3 & 6 & 1 & -0.46 & 3.68 \\ \text { Overall } & 1.49 & 1 & 0.96 & 0.22 & 5 & 1 & -0.19 & 3.17\end{array}$

Action 4: Linking hashtags

\begin{tabular}{lcccccccc} 
Easy to do & 2.89 & 2 & 2.02 & 0.46 & 7 & 1 & -2.99 & 8.77 \\
Time avaliable & 2.79 & 3 & 1.51 & 0.35 & 6 & 1 & -0.29 & 5.87 \\
Info support & 2.94 & 2 & 2.18 & 0.5 & 7 & 1 & -2.97 & 8.86 \\
Overall & 2.87 & 2.33 & 1.76 & 0.4 & 6 & 1 & -1.75 & 7.49 \\
\hline
\end{tabular}

* 'Max' refers to a category's highest score denoting dissatisfaction.

$\dagger$ 'Min' refers to a category's lowest score denoting satisfaction.

$\ddagger$ 'Overall' is an average of the three questions in the ASQ questionnaire. 
it came first on top of the search results in the Hashtag Linking View and its title matches exactly the hashtag's text (see Figure 3.5 for reference). Partially similar to it is hashtag '\#programming' which appeared on the first page of the results but with a small caveat that its corresponding article appears on the bottom of the list with a slightly different title of "Computer programming." For the hashtag "\#angry_birds," however, we find that many students had linked the wrong Wikipedia articles for some reason we do not understand.

Table 5.4: Results from the Wilcoxon rank test.

\begin{tabular}{lcc}
\hline Pair & $p$-value & $\mathrm{V}$ \\
\hline PnP-OneNote & 0.002 & 195.0 \\
PnP-NotesKB & 0.025 & 146.5 \\
OneNote-NotesKB & 0.434 & 65.5 \\
\hline
\end{tabular}

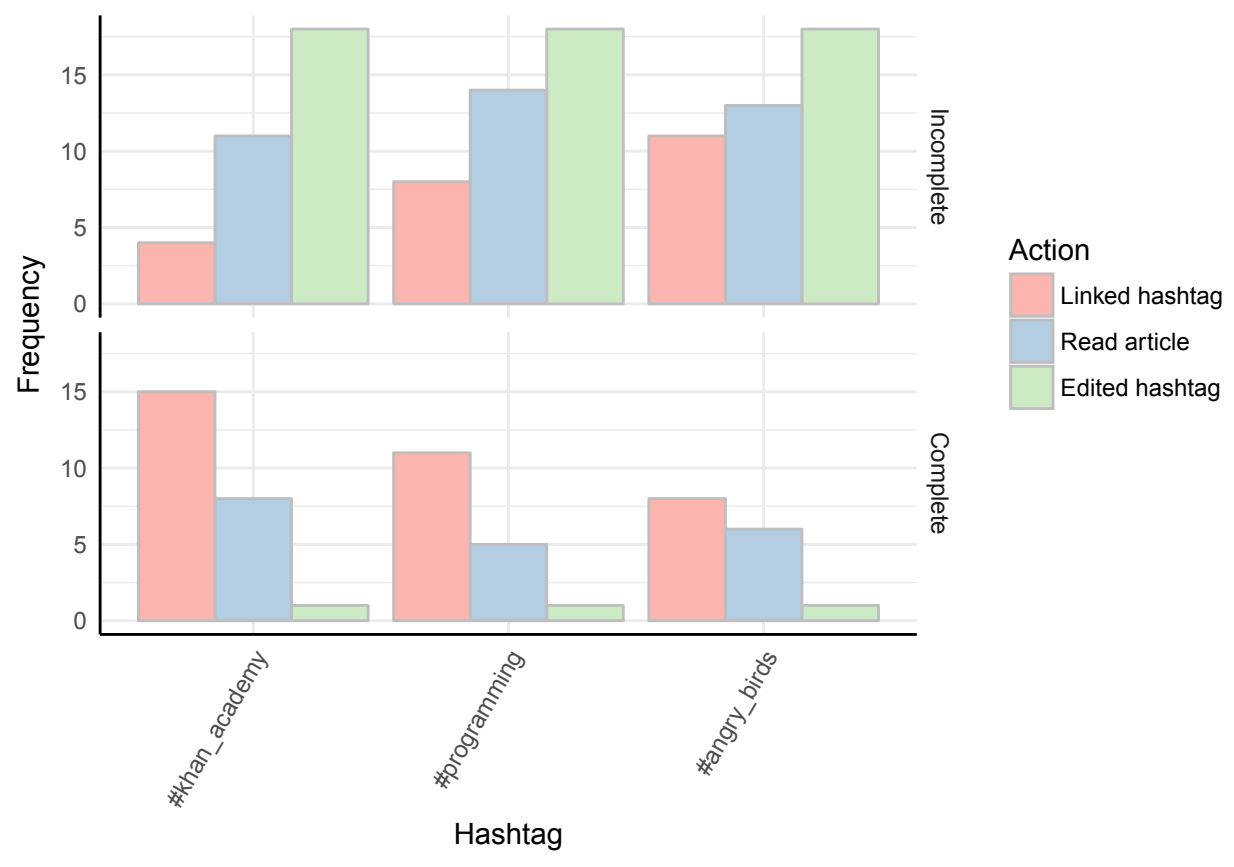

Figure 5.8: Barplots show the completion rate by participants carrying out the Wikification process as part of Action 4. 


\subsubsection{Qualitative Results}

Using the thematic analysis for the qualitative aspects of our study, we identified three different themes drawn from the comments and remarks made by participants. Achieving that required analyzing 12 hours of screen captured videos and transcribing over 10-15 minutes of interview time per participant. As explained in the earlier chapter (see Section 4.3.4), the interviews were conducted toward the end of the testing session and they were intended to outline and record the general perceptions of participants annotating online educational videos using the three different methods. When identified four general themes that encapsulate the majority of our findings:

1. Effect on note-taking practices.

2. Easiness and Power.

3. Task familiarity and activity support.

4. Content style effects on outcomes.

\subsubsection{Theme 1: Effect on note-taking practices.}

This is thought to be a characteristic of the user that is independent of the annotation method that was in use. For example, making some drawings and connections around one's notes. A quote from one of the participants best illustrates this "I am not very organized with the paper... I will scribble like a huge arrow from one side to the other, and then if there's something that I forgot or need to change, I will cross out, and I will continue to add around the text." Another example is addressing writing in a short and a point-by-point form by Participant-020 "if you do not want to write out... a word by word... you sometimes have to think you know, a short form method it will make it, you know, [it is] easy for you to tell what was wer.. - what was being written when you make it in point form." 


\subsubsection{Theme 2: Easiness and Power.}

This theme is concerned with how much power one annotation method does provide to its users, versus its ease of use. Many have noted that they can do something using one method (usually PnP) but not with another. This is usually manifested in mentions of one's familiarity to use one method and the need for him/her to learn how to operate it, versus the power it gives them in comparison to another. It all boils down to the measurement of learnability and the usability of a note-taking method. For example, a participant has mentioned that specifying annotation-types in OneNote was harder to do than in NotesKB. A similar thing can be found in a quote by Participant-006 talking about choosing between $\mathrm{PnP}$ or NotesKB as the most preferred method:

"Well I am torn, [be]cause pen and paper is easier to, sort of, grow something organically, right. So, I can write my thoughts really quickly, annotate it whenever I want, like underline things whenever I want. Whenever I am using anything that requires typing, then I have to have a plan, right. I have to usually annotate it at the start, and if I change my mind, then I have to grab the mouse and then re-click to change the annotation, which is problematic."

Several testimonies expressed similar things to that by other participants as well. On the other hand, many participants had praised software methods (OneNote and NotesKB) for their ability to automate things provide some assistance by the computer; especially in spelling checking, copy/paste mechanism, text indexing, and fast typing. A common criticism that is shared by both OneNote and NotesKB, though more by the latter; has to do with the size of the video displayed within the tool as being too small to see.

\subsubsection{Theme 3: Task familiarity and activity support.}

This theme involves the method's ability to support the note-taking activity in general, and in the HiForm annotation style in particular. Under this theme, we include 
comments made by participants during interviews about their experience during the annotation sessions. For instance, so indicated that looking at the video, thinking about the content, and taking notes of it all at the same time was quite challenging for them. Being familiar with the tool and the task, in general, is a point that had appeared again and again in the interviews. The following quote is an example: "It [NotesKB] just need a bit of familiarization I think." Another related point to familiarity, is one that deals with task interleaving. As Participant-001 put it: "I just can't continue to listen and type without pausing the video and figuring out what type of comment I was writing." On the contrary, Participant-010 had praised NotesKB saying: "I was not really getting distracted to watch video; I was just listening and writing." A similar remark to that was made about subtitles and their importance as expressed by an international student whom English is a second language. The gist of the remark revolves around the time it takes a second language speaker to grasp all the information presented in the videos and the need to sometimes repeated more than once (Video-B lack subtitles). Others had mentioned that NotesKB felt more like a transcribing tool rather than a tool for learning. When it comes to Wikification, there was mostly positive feedback. A quote from Participant-011 states "I like the hashtags - like you can link to Wikipedia articles like that was really nice." A similar point addressed by another participant "if I am hashtagging then I have to hashtag while I am typing or again interrupt the flow of my thought to hashtag something. So it is. . . I think overall I still prefer pen and paper."

\subsubsection{Theme 4: Content style effects on outcomes.}

Many participants mentioned that the simplicity of Video-A had caused them to take fewer notes than they had intended; while the complexity of Video-B (and sometimes Video-C) and the many historical facts presented in it has resulted in them taking more notes. For instance, Participant-013 mentioned that "I had to like write more for the last one but the first one [VideoA], like Proper Noun, like nouns in general and examples. So I did not really have to write so much." The fact that no one had chosen Video-A as being the longest nor that it has enough material to take notes 
about is a point that was repeatedly raised by some participants. Linked to that, participants also mentioned that their familiarity with the content of a video had in a way determined the quality and quantity of taken annotations. Moreover, some have stated that the use of animations and the pace speed in which the video plays was of importance to some students.

\subsection{Interpretation and Discussion}

Considering all the results of our study, we realized there were several interrelated qualities that affect the note-taking experience. From the recorded observations and findings during the Note-taking Sessions, the following lists these qualities:

- Annotation Method Support - concerned with the level of satisfaction the students felt toward the assistance an annotation method provided during the annotation session.

- Annotation Style - concerned with the annotation style was used during the note-taking session, which was the same for all of the three sessions (HiForm style).

- Learning Content - concerned with the learning content presented during the session.

- User Competence - concerned with how competent the student was to carry out the learning task, which is determined by how well they were doing academically as well as the general skill sets they obtain.

\subsubsection{Annotation Method Support}

To give an overview of the principal obtained results, we show in Table 5.5 and Table 5.6 summaries of the SUS and ASQ tests respectively. The reported SUS scores show how the three annotation methods' usability (PnP, OneNote, and NotesKB) were perceived by the participants after they have been asked to use them to take notes of online educational videos. As expected, when compared to other methods, PnP 
Table 5.5: Summary of SUS scores.

\begin{tabular}{|c|c|c|c|c|c|c|c|c|c|}
\hline & \multicolumn{9}{|c|}{ Annotation Method } \\
\hline & \multicolumn{3}{|c|}{$P n P$} & \multicolumn{3}{|c|}{ OneNote } & \multicolumn{3}{|c|}{ NotesKB } \\
\hline & $\begin{array}{c}\text { SUS } \\
\text { Score } \\
\end{array}$ & Usability & Learnability & $\begin{array}{c}\text { SUS } \\
\text { Score }\end{array}$ & Usability & Learnability & $\begin{array}{c}\text { SUS } \\
\text { Score }\end{array}$ & Usability & Learnability \\
\hline Avg. & 80.9 & 79.1 & 88.1 & 55.9 & 51.6 & 73.1 & 62.2 & 62.7 & 60.6 \\
\hline
\end{tabular}

Table 5.6: Summary of ASQ scores.

\begin{tabular}{ccccc}
\hline & \multicolumn{4}{c}{ Actions } \\
\cline { 2 - 5 } & Action 1 & Action 2 & Action 3 & Action 4 \\
\hline Avg. & $\mathbf{3 . 4}$ & $\mathbf{1 . 3}$ & $\mathbf{1 . 5}$ & $\mathbf{2 . 9}$ \\
\hline
\end{tabular}

always come on top as the most usable method. However, as we are mostly concerned with software tools, the SUS scores of OneNote and NotesKB are truly the ones in question in this thesis. The reason for that is simple, using $\mathrm{PnP}$ to annotate videos is not possible, at least in the foreseeable future, of producing notes that are semantically linked. As mentioned earlier, its presence is to act as a catalyst for participants to be encouraged to take notes in the HiForm style. Based on the ASQ scores, we notice that most participants found Action 2 and Action 3 to be the most satisfactory.

\subsubsection{Annotation Style}

As mentioned earlier, NotesKB was built to fit the HiForm style of taking notes, and we influenced students to adopt it in the other two methods (namely PnP and OneNote). However, that style did not suit all of the students. Some students pointed out that they did not like taking notes in a point-by-point form. Many others have pointed out that they chose $\mathrm{PnP}$ just for the freedom it gives them to make some drawings very quickly. Nevertheless, initially, when we started planning for this study, we thought that the study would involve students that already know how to take good notes using an established paradigm. As it turned out, they were different in the ways they take their notes, and they seem to need more time to adjust to using a new note-taking paradigm. Contrary to expectation, it seems that the students' familiarity 
using social networks did not necessarily translate to them using the HiForm style at a higher rate. We initially had projected that when it comes to writing notes in the HiForm style, the high usage of the social networks by participants will manifest itself in a higher style adoption rate, which has proven to be inaccurate. That does not say there is no relation between the two, but our findings suggest that there are no evident connections between them. Switching between contexts exemplified in utilizing some writing elements and practices gained from networking on social media and put them into practice in an e-learning context is found to be more complicated than we had initially thought. Another interesting observation is that we found that many participants specified hashtags and annotation-types after they had finished writing them. That is to say many had tried to incorporate different parts of the style into their notes after they had finished taking them as opposed to adopting it in real-time.

\subsubsection{Learning Content}

Similar to that, some participants did not remember what video was the longest in duration. That suggests that coding perceived information does not necessarily result in the recollection of it. That is to say, just because a learner was exposed to some educational contents and he/she had actively interacted with in some form, annotating it as in this case; that does not determine nor lead to recollecting those encoded thoughts. In that regard, it can also be said that the information density of a video's learning contents resulted in the participants perceiving it as being longer than it actually is. Even though we have told the participants before watching each video about the time they have available to them to annotate a video, that did not prevent some of them to mistakenly assume that Video-B was the longest. We reasoned that knowing the videos' perceived length could in some way indicates the density of the information presented; so more participants thinking a video to be the longest, the more likely to be dense.

When it comes to the nature of the selected videos, they were of three types, a conceptual, a factual and one that is a mix of both. With such characterization, 
we think our results cannot be generalized and include other educational videos that are of different content types and styles. For example, educational videos that include educational contents of procedural nature (e.g., computer programming) or an analytical one (e.g., mathematics) are not tested in this study. Thus there is a possibility of different results to emerge.

\subsubsection{User Competence}

The competence of the participants is best illustrated in the data collected about their academic backgrounds and their abilities to use computers and their level of familiarity using social networks. We observed from the conducted interviews as well as from analyzing the notes they took, the less skilled and academically advanced a student is, the less the amount of annotations is taken by them, regardless of the difficulty of the learning content. On the other hand, a user that is highly competent in their general skills, as well as academically, would take more notes and have more video watching time.

Moreover, for Wikification, we speculate that the reason for the non-completion of the linkage process of connecting Wikipedia articles to hashtags by some participants in Action 4, is due to either confusion or indifference. Many of those who had failed to complete linking the intended articles seemed to be fixed on selecting the results that were displayed first. For instance, a common mistake was found in linking the hashtag "\#angry_birds." The article that rightly corresponds to that hashtag did not appear to the user until he/she had visited all pages leading up to the fourth search results page. Therefore, some participants settled on choosing either the "Angry Birds Seasons" article or the article adjacent to it; the "Angry Birds Go!"; which both had appeared on the first page of the results. There is another possibility to consider, though unlikely, that is the fatigue effect was experienced by participants, given that Action 4 took place by the end of the study time. 


\subsubsection{Reflections}

Upon reflection on the discussed four qualities, we think that the students' split attention between the video, annotation style, and the tool was key to understand the difficulty that students faced during their note-taking session using the NotesKB tool. We are inclined to think that asking students to watch a video, use a specific annotation style, and interact with a new tool with which have a limited familiarity dealing with all at the same time; can be simply described to be overwhelming to the students' cognitive load. That, in turn, may have had a direct implication on the overall interaction experience. A suggestion to help in that regard, is that facilitating the pausing of the video by using the keyboard, via some shortcut, may be of be of help to the students. In this way, when the student become overloaded they could pause the video while continuing in taking notes.

Although learning outcomes were regarded as being out of scope in our study, from conducting the study we had gained some understanding of the learning that took place. On the one hand, some participants asked during the interviews to be reminded of the videos they have just watched, had a misapprehension about the videos' length or have shown clear signs of disinterest learning from the videos. On the other hand, other students, especially competent ones, have shown some appreciation toward the videos by describing them to be interesting, took repeated pauses of videos and/or re-watched them to make careful and well thought-out notes, as well as suggestions some improvements to the tool or the study in general. Despite that, we think those are just subjective impressions and it is hard to be conclusive about them. 


\section{Chapter 6}

\section{Conclusion}

To recap, this thesis project is about building a principled e-Learning application with a usable UI to annotate educational videos, called NotesKB; then testing its usability by comparing it to other note-taking methods that are frequently used by students. The tool is designed to be used for annotating online multimedia learning materials and leveraging ontologies available on some LOD cloud services such as DBpedia. These ontologies would be integrated into the text in the form of hashtags, which are entered by the user during an annotation session. From comparing the usability of the three different note-taking methods (PnP, OneNote, and NotesKB) using some standardized usability tests (SUS and ASQ), we found that PnP is most usable and there is no significant difference between our tool (NotesKB) and OneNote. Having said that, we add that it is possible that the design of a software note-taking tool with semantic annotation capabilities to be comparable, in terms of usability, to a widely-used one without, namely OneNote. We also think that such tool is potentially valuable to the students in the long run as their notes will be machine-readable making them easy to search and retrieve. Testing the satisfaction level of the semi-automatic semantic linking mechanism fused with epistemic action was shown to be satisfactory by most students according to the ASQ test results. Information retrieval is one of the sought advantages. NotesKB should be easily installed into the Chrome web browser as an extension and use it to annotate generic Web videos and address annotations at different levels of complexity and expressivity. 


\subsection{Research Outcomes}

\subsubsection{Main Findings}

Since there is no significant difference between OneNote and NotesKB regarding usability as the SUS scores show, it is helpful to know that most students participated in the study picked NotesKB as their preferred method of annotation. This study gives a glimpse and provides a soft evidence of the existing potential of using and incorporating various SW technologies into developing more tools suitable for students, as we think the responsibility of supporting taking good notes falls on the tool design. Although it is unclear how to teach students or induce them to adopt good note-taking practices, as they can be learned independently; this study forms an exercise in which we argue that annotation methods do affect the annotation process similar to how the learning content does. Given the various stages that this project went through from initially conceiving the idea of the NotesKB tool, to fully designing it and building it; we realized that reducing cognitive overload is the most critical element when building such a tool for students. Thus, since the first design draft of NotesKB, we geared our efforts to test this particular relationship between annotation style and tool structure. Choosing educational videos as a learning medium determined by the weak support for this medium. This is despite the medium being widespread in the current educational scene as a way for educators to present their contents on Web platforms such as YouTube.

We found that the process of note-taking is more complex than we had initially thought at the start the project. Before all else, the learning tool (in our case the annotation method) affected the learning experience, because the degree of support and assistance that a tool provides has impacted the user's performance and level of satisfaction carrying out the learning task. We also found that being familiar with the annotation method is also important. It is wrong to assume that because students use software $x$, therefore, they will know how to use software $y$. Despite the fact that the majority of our participants, whose education was above average and use social media at least once a day (Facebook most probably); taking notes using NotesKB was not that 
straight forward to them. One reason for this is that the context is different from that of social media. Sharing a post on social media, for the most part, requires little to no cognitive processing, unlike learning something from an educational video. Second, some participants pointed out that they did not have enough time to come back and edit their notes, enter hashtags and change annotation-types; and as we discovered, students usually do these editing-related types of task toward the end of an annotation session.

\subsubsection{Limitations}

Given the scope of our study, we do not think our findings can be generalized. First, the number of our participants was small. We might end up with different results had we had more participants. Also, the participants' demographics were quite skewed as there were more males than females; more graduate than undergraduates or postgraduates; and more students enrolled in technical programs than non-technical ones. Second, the nature of each presented video was different from the other two videos. Some participants were not interested in learning about some of them, and some participants have even expressed their annoyance and pointed out that they were given a little amount of time to watch and annotate these videos. Moreover, as mentioned before, the sequence order in which students experienced the annotation methods (PnP followed by OneNote then NotesKB) was the same for both testing groups, and the only difference was in the videos being watched using them. Given that, we do not know how the results may differ had we changed that order and whether or not new results may emerge out of this change. We also think that the difference in the sizes of videos and the space they occupy of the screen had made analyzing and comparing the collected results challenging and impracticable. Lastly, giving participants enough time to try out each annotation method before the note-taking session was another limitation in our study, especially for NotesKB. Participants had the chance to only watch demo videos without trying the method out for themselves. 


\subsection{Contributions}

- Principled design of an e-Learning video annotation system.

- Usability comparison of three different annotation methods, namely PnP, OneNote and our tool NotesKB.

- Conceptualizing the different factors effecting the students' note-taking process of online educational videos, and understanding the relationships between them.

\subsection{Future Work}

Future work should investigate the determining factors involved in annotating different kinds of multimedia learning contents and not restricted to videos only. Also, the study should include other annotation methods than the ones we tested. Further, the study should be conducted with students in different age groups and levels to see if that would affect the findings. 


\section{Appendix A}

\section{Annotation-Types}

For each annotation-type presented below in Table A.1, we display its visual symbol along with a short description of its nature; that is borrowed primarily from the Oxford Dictionary of English [89].

Table A.1: Descriptions of the underlying meaning of annotation-types.

\begin{tabular}{|c|c|c|}
\hline \multicolumn{2}{|c|}{ Symbol } & Description \\
\hline Txt & Text & $\begin{array}{l}\text { This is the main block in which all blocks are derived } \\
\text { from. }\end{array}$ \\
\hline C & Concept & $\begin{array}{l}\text { A word or phrase used to describe a thing or to } \\
\text { express a concept, especially in a particular kind of } \\
\text { language or branch of study. }\end{array}$ \\
\hline Def & Definition & $\begin{array}{l}\text { A statement of the exact meaning of a word, } \\
\text { especially in a dictionary. }\end{array}$ \\
\hline$?$ & Question & $\begin{array}{l}\text { A sentence expressed as an inquiry so as to draw out } \\
\text { a reply. }\end{array}$ \\
\hline Ans & Answer & A reply to an asked question. \\
\hline Eg & Example & $\begin{array}{l}\text { A thing characteristic of its kind or illustrating a } \\
\text { general rule. }\end{array}$ \\
\hline$\neg \mathrm{Eg}$ & Counter Example & $\begin{array}{l}\text { An example that opposes or contradicts an idea or } \\
\text { theory. }\end{array}$ \\
\hline $\mathrm{Ag}$ & Argument & $\begin{array}{l}\text { A set of reasons advanced for or against a proposal } \\
\text { or proposition (i.e. providing reasons to believe). }\end{array}$ \\
\hline$\neg \mathrm{Ag}$ & Counter Argument & $\begin{array}{l}\text { An argument or set of reasons put forward to oppose } \\
\text { an idea or theory developed in another argument. }\end{array}$ \\
\hline "Q" & Quote & $\begin{array}{l}\text { Repeat or copy out (words from a text or speech } \\
\text { written or spoken by another person). }\end{array}$ \\
\hline
\end{tabular}


Appendix B

Pre-Test Questionnaires 


\section{Demographic and Background Information}

\section{Demographic and Background Information}

Age *

Please write your answer here:

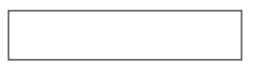

Gender *

Please choose only one of the following

Male

Female

Prefer not to say

I consider myself:

Please choose only one of the following:

Right-handed

Left-handed

Onbidextrous

What is your current academic field of study?

Please write your answer here:

What is the level of your current academic program?

Please choose only one of the following:

Undergraduate

Graduate

Other

How would you rate your academic performance? *

Please choose only one of the following:

Struggling student

Below average student

Average student

Above average student

Excellent student

Please evaluate your familiarity with the following computer-related activities:

Please choose the appropriate response for each item:

Web browsing

Not at all Unfamiliar

O

Somewhat

O

Familiar

Very Familiar 


\section{Demographic and Background Information}

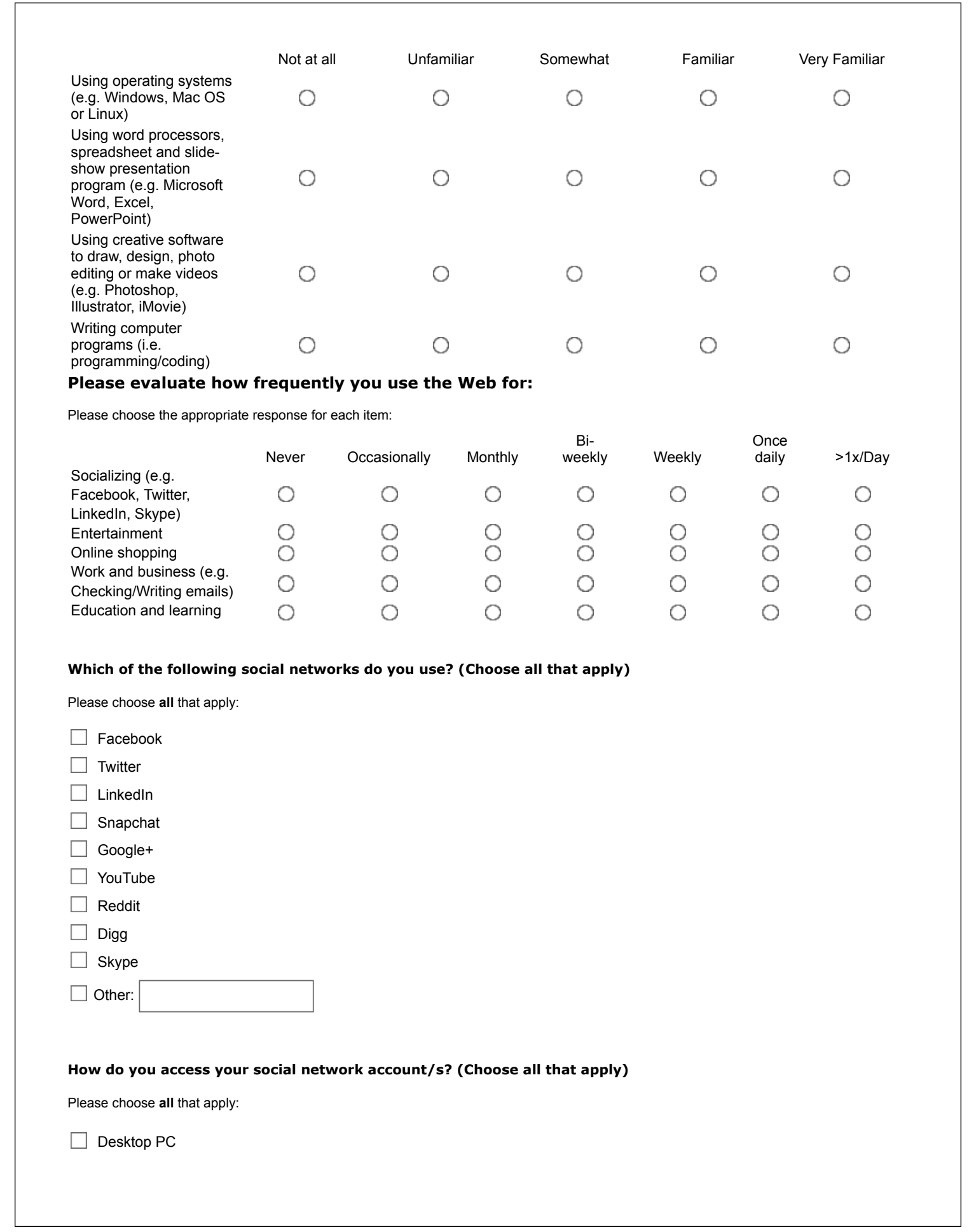




\section{Demographic and Background Information}

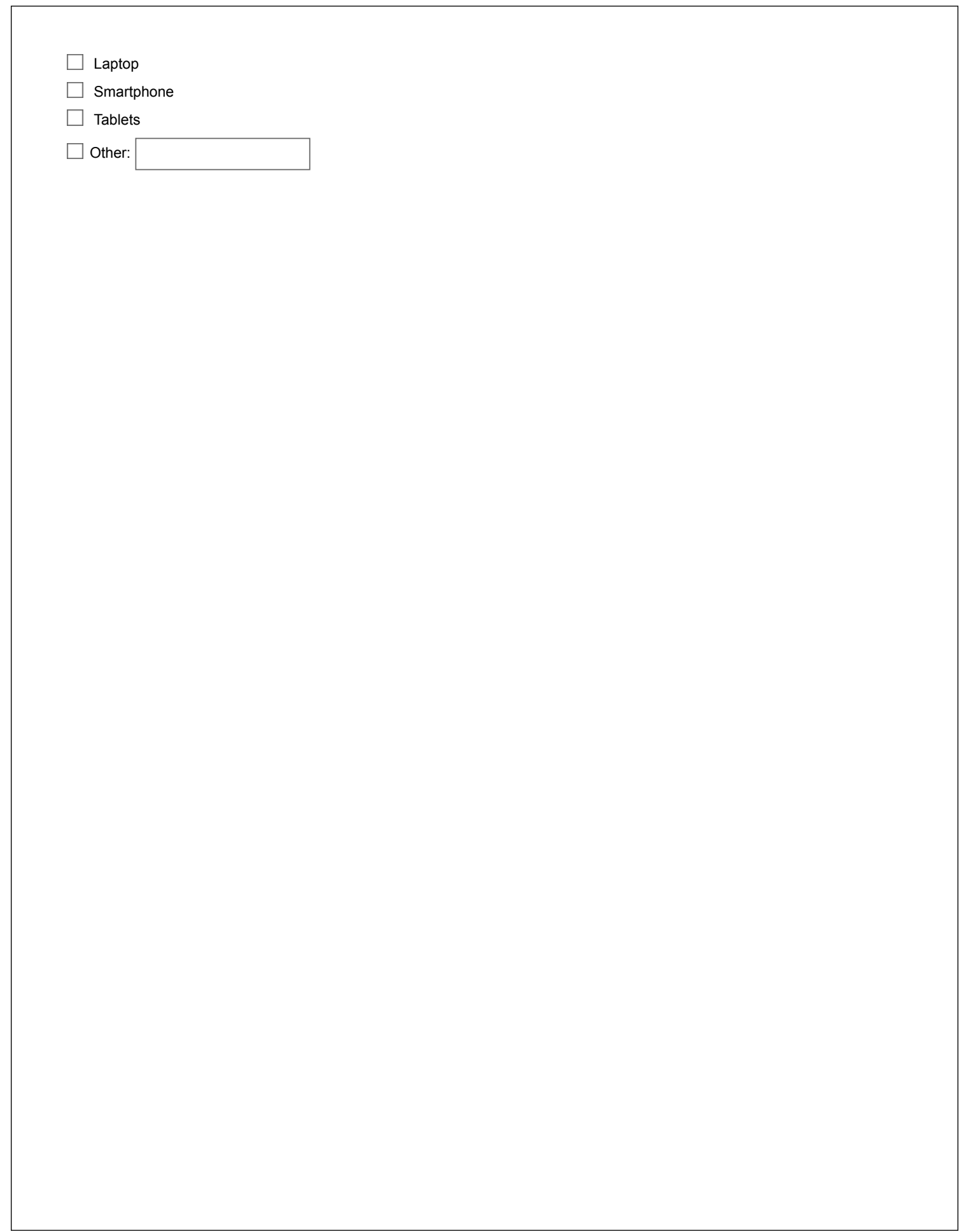




\section{Educational and Learning Activities}

\section{Educational and Learning Activities}

\section{As a student, do you anticipate to:}

Please choose the appropriate response for each item:

Enrolling in a more advanced degree from the one you are currently studying

Changing your current field of studying

Pursuing a career after graduation in a different field from the one expected of someone of your

academic background

How many hours do you spend on average on a weekly basis on the following:

Please choose the appropriate response for each item:

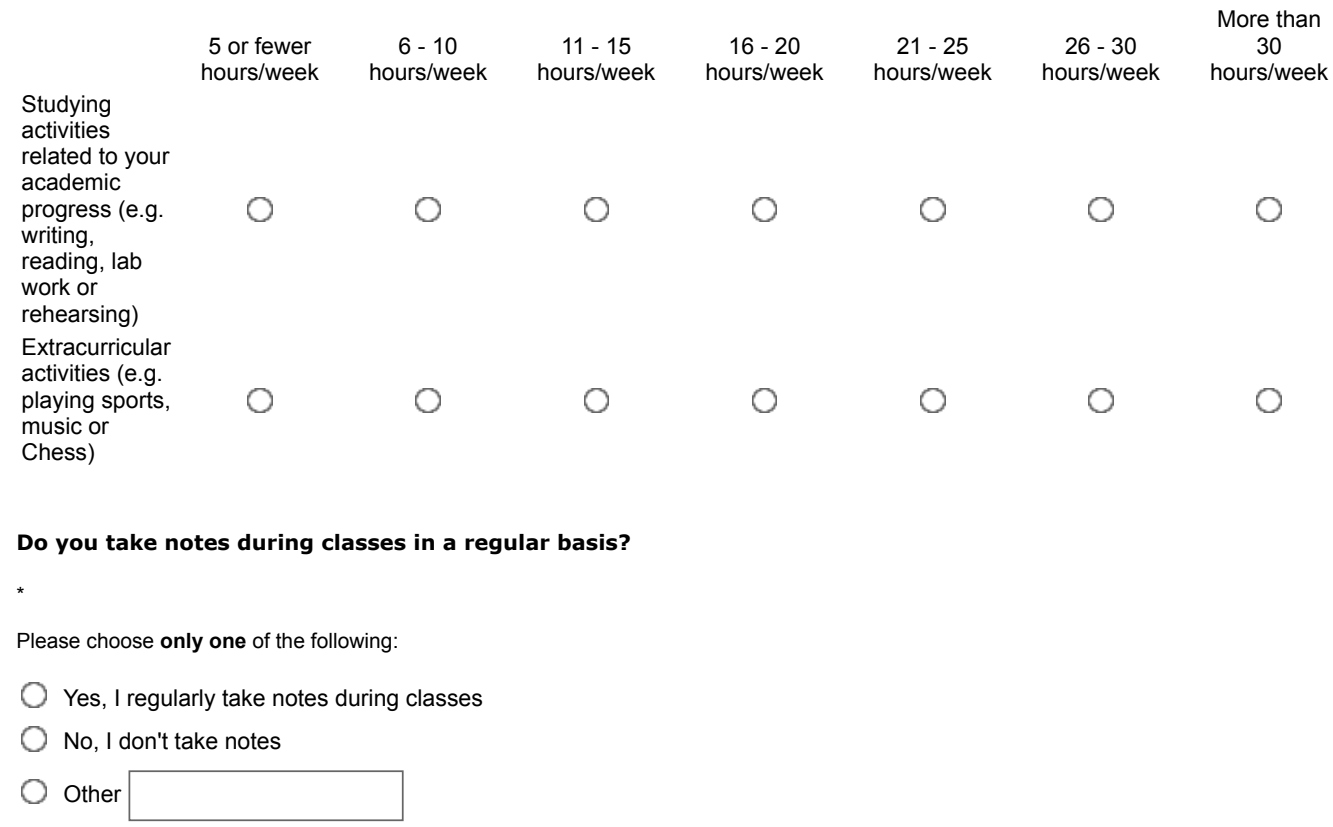

What medium do you use often to take your class notes?

Only answer this question if the following conditions are met:

Answer was 'Yes, I regularly take notes during classes' at question '14 [q3notetakinghabit]' (Do you take notes during classes in a regular basis? )

Please choose only one of the following

Paper

Recording

Electronic

Other

On a scale of 1 to 10 , rate the quality of the following aspects of your note taking habits: 


\section{Educational and Learning Activities}

Only answer this question if the following conditions are met:

Answer was 'Yes, I regularly take notes during classes' at question '14 [q3notetakinghabit]' (Do you take notes during classes in a regular basis? )

Please choose the appropriate response for each item:

\begin{tabular}{|c|c|c|c|c|c|c|c|c|c|c|}
\hline & $\begin{array}{c}\text { Poor } \\
1\end{array}$ & 2 & 3 & 4 & 5 & 6 & 7 & 8 & 9 & $\begin{array}{c}\text { Excellent } \\
10\end{array}$ \\
\hline $\begin{array}{l}\text { Organization of your } \\
\text { notes }\end{array}$ & 0 & 0 & 0 & 0 & 0 & 0 & 0 & 0 & $\checkmark$ & 0 \\
\hline $\begin{array}{l}\text { Recognizing main } \\
\text { ideas when revisiting } \\
\text { your notes }\end{array}$ & 0 & 0 & 0 & 0 & 0 & 0 & & 0 & & 0 \\
\hline $\begin{array}{l}\text { Recognizing } \\
\text { supporting details of } \\
\text { main ideas when } \\
\text { revisiting your notes }\end{array}$ & 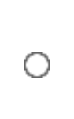 & $c$ & & 0 & 0 & & & 0 & & \\
\hline $\begin{array}{l}\text { Recognizing } \\
\text { reoccurring patterns } \\
\text { in your notes (i.e. } \\
\text { cause-effect, } \\
\text { concept-definition, } \\
\text { example) }\end{array}$ & 0 & 0 & & & 0 & 0 & & & & \\
\hline $\begin{array}{l}\text { Integrating your } \\
\text { notes with other } \\
\text { sources (e.g. } \\
\text { readings or another } \\
\text { colleague's notes) }\end{array}$ & 0 & 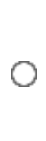 & $r$ & 8 & 0 & & & & & \\
\hline $\begin{array}{l}\text { Summarizing your } \\
\text { notes using your } \\
\text { own words }\end{array}$ & 0 & 0 & 0 & 0 & 0 & 0 & & & & \\
\hline $\begin{array}{l}\text { Conducting regular } \\
\text { reviews of your } \\
\text { notes }\end{array}$ & 7 & 0 & 0 & 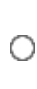 & 0 & 0 & & 0 & 0 & 0 \\
\hline $\begin{array}{l}\text { Editing your notes } \\
\text { within } 24 \text { hours after } \\
\text { class }\end{array}$ & 0 & 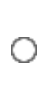 & 0 & & 0 & 0 & & & & \\
\hline
\end{tabular}

How often do you watch online educational videos (e.g. on Carleton University OnLine, Khan Academy, Coursera, edX, etc)?

$*$

Please choose only one of the following

Never

Occasionally

Monthly

Bi-weekly

Weekly

Daily

$>1 x /$ Day

Which platform/website have you used to watch educational videos, if ever? (Choose all that apply)

Please choose all that apply:

$\square$ Carleton University OnLine (CUOL) 


\section{Educational and Learning Activities}

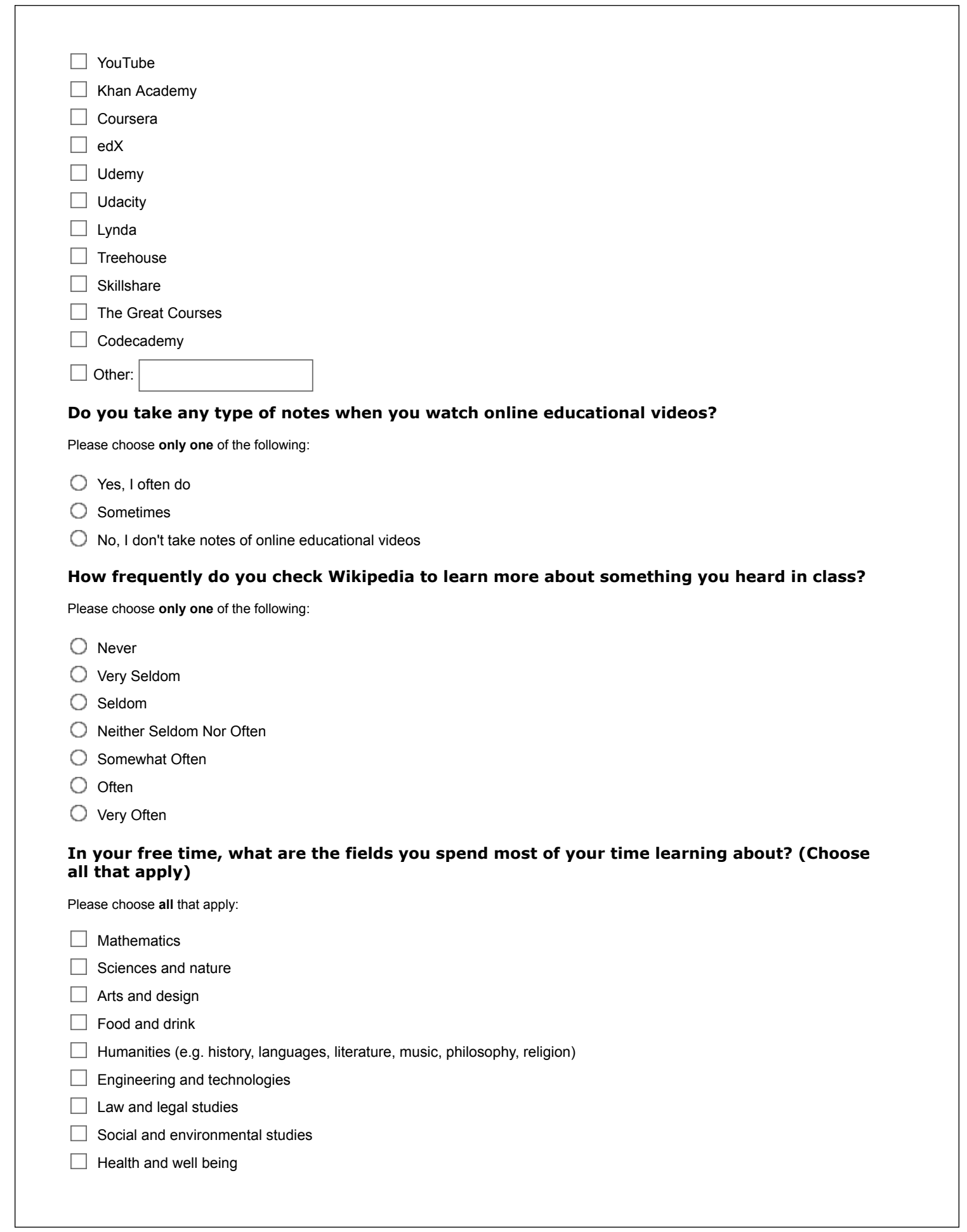




\section{Educational and Learning Activities}

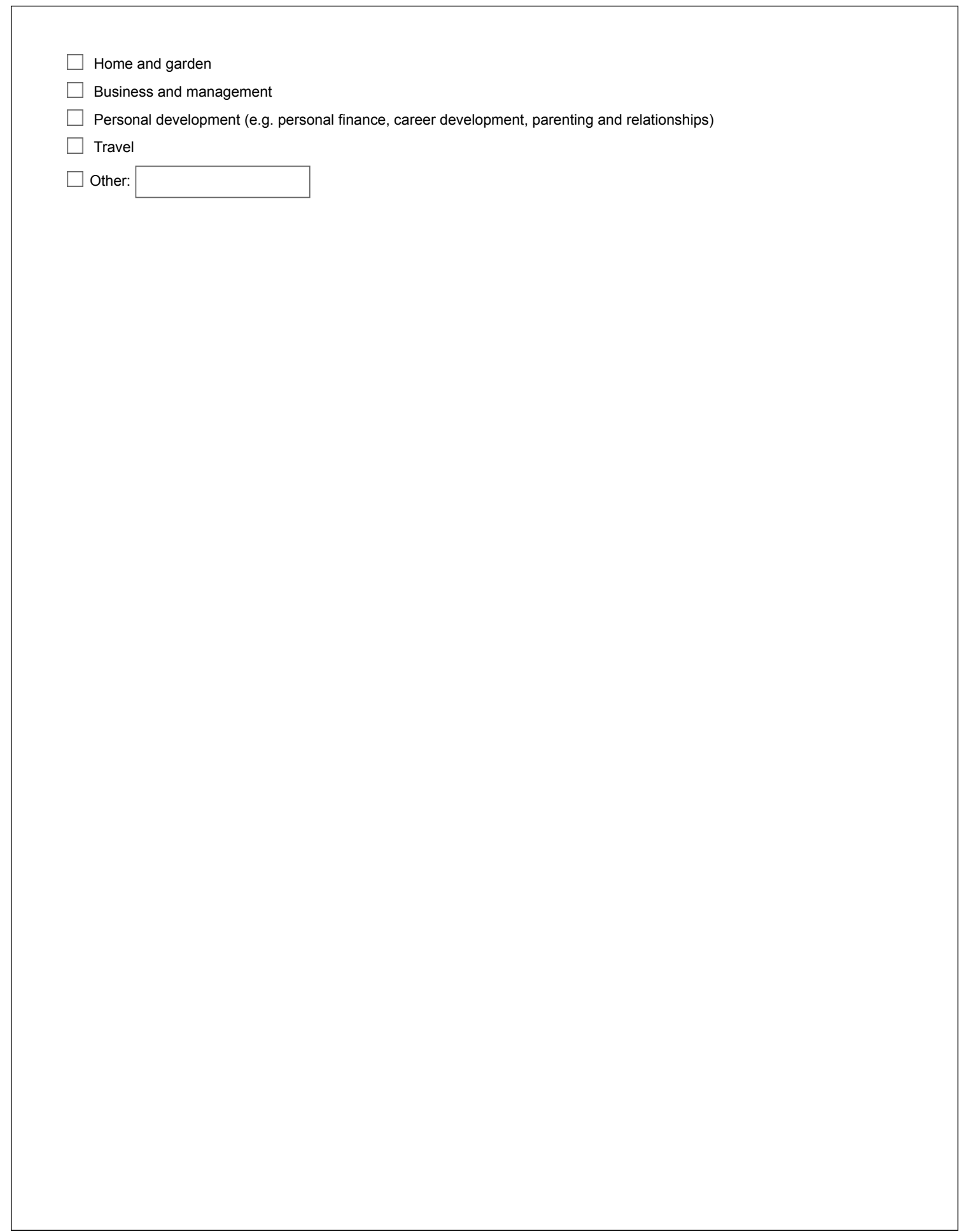


Appendix C

Post-Test Questionnaires 


\section{System Usability Scale (SUS) - Post-study Usability Test}

\section{Video \# 1: Introduction to nouns}

In this task, we would like you to take notes about an educational video using pen \& paper.

\section{Task Instructions}

Please use the follwoing instructions below to carry out the task:

- You have 10 minutes to watch the video and take notes about it.

- Make sure to include the video's title, website name and author.

- While annotating (i.e. taking notes), try to identify every proper noun by underlining it. For example:

"This video is related to English Grammar".

- Moreover, beside each new point you record, try to indicate what it is about in your notes (e.g. using "def." for a defnition, "Q:" for a question, or "e.g." for an example, etc). [Please refer to the provided list of annotation types]

\section{Video to Annotate}

Link of educational video: https://youtu.be/UeiW-WQpuis

Please select the number that best describes your experience using a pen \& paper as a method of taking notes:

$*$

Only answer this question if the following conditions are met:

Answer was 'Yes' at question '22 [t3finished]' (Have you finished watching and annotating the video?)

Please choose the appropriate response for each item:

Strongly

Disagree

think that I would like to use the pen \& paper

frequently to take notes.

I found the pen \& paper

unnecessarily complex.

I thought the pen \& paper were easy to use.

I think that I would need the support of a technical person to be able to use this method.

I found the various functions in this method

were well integrated.

I thought there was too much inconsistency in

this method.
1

0

0

0

0

0

0
2

0

0

0

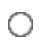

0

0
3

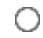

0

0

0

0

0
Strongly Agree

4

5

0

0

0
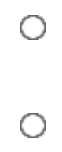


\section{System Usability Scale (SUS) - Post-study Usability Test}

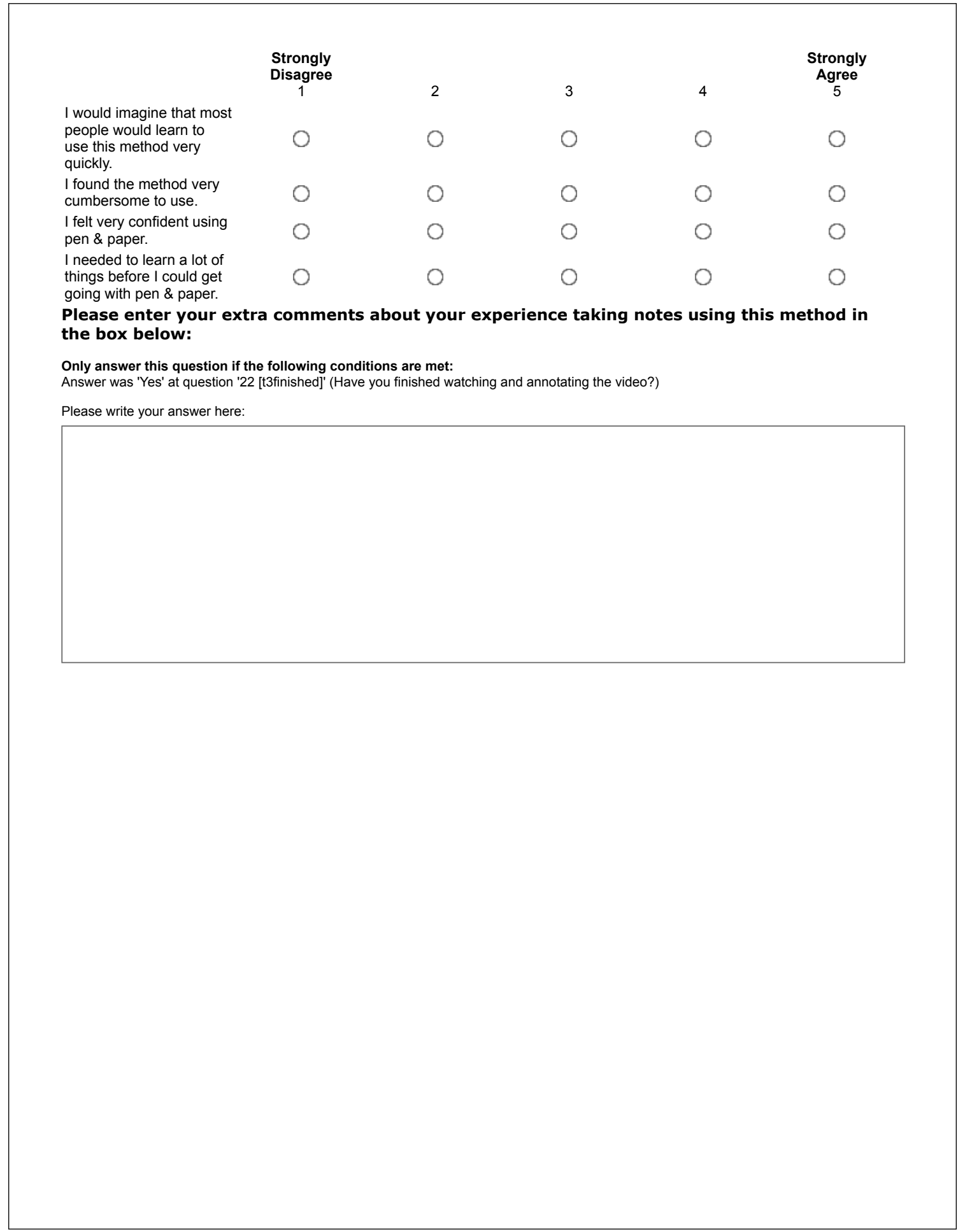


System Usability Scale (SUS) - Post-study Usability Test

\section{OneNote Demo}

Please check the link below, to give you an introduction of how to use Microsoft OneNote application to take notes of an online educational video:

Please click on the follwoing link to watch the demo: Preview

Have you found the demo useful?

Please choose only one of the following:

Yes

No 


\section{System Usability Scale (SUS) - Post-study Usability Test}

\section{Video \# 2: Firestick farming}

In this task, we would like you to take notes about an educational video using the OneNote tool.

\section{Task Instructions}

Please use the following instructions below to carry out the task:

- You have 15 minutes to watch and annotate the video.

- Make sure to include the video's title, website name and author.

- Take notes about (i.e. annotate) the video; and try to type down every proper noun you identify as a hashtag (precede it by the '\#' sign). For example: "This video is about \#Firestick_farming".

- Moreover, beside each new point you record, try to indicate what it is about in your notes (e.g. using "def." for a definition, "Q:" for a question, or "e.g." for an example, etc).

\section{Video to Annotate}

Link of educational video: $\underline{\text { https://youtu.be/mwUyaeWxJhA }}$

Please select the number that best describes your experience taking notes using the OneNote tool:

$*$

Only answer this question if the following conditions are met:

Answer was 'Yes' at question '26 [t2finished]' (Have you finished watching and annotating the video?)

Please choose the appropriate response for each item:

Strongly

Disagree

2

I think that I would like to use this software

frequently.

I found the software unnecessarily complex.

I thought the software

was easy to use.

I think that I would need

the support of a technical person to be able to use this software.

I found the various functions in this software were well integrated.

I thought there was too much inconsistency in this software.
2

$0 \quad 0$

$0 \quad 0$

0

0

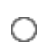

0
3

0

0

0

0

0

0
Strongly

Agree

4

5

0

O

O

O

O

0 


\section{System Usability Scale (SUS) - Post-study Usability Test}

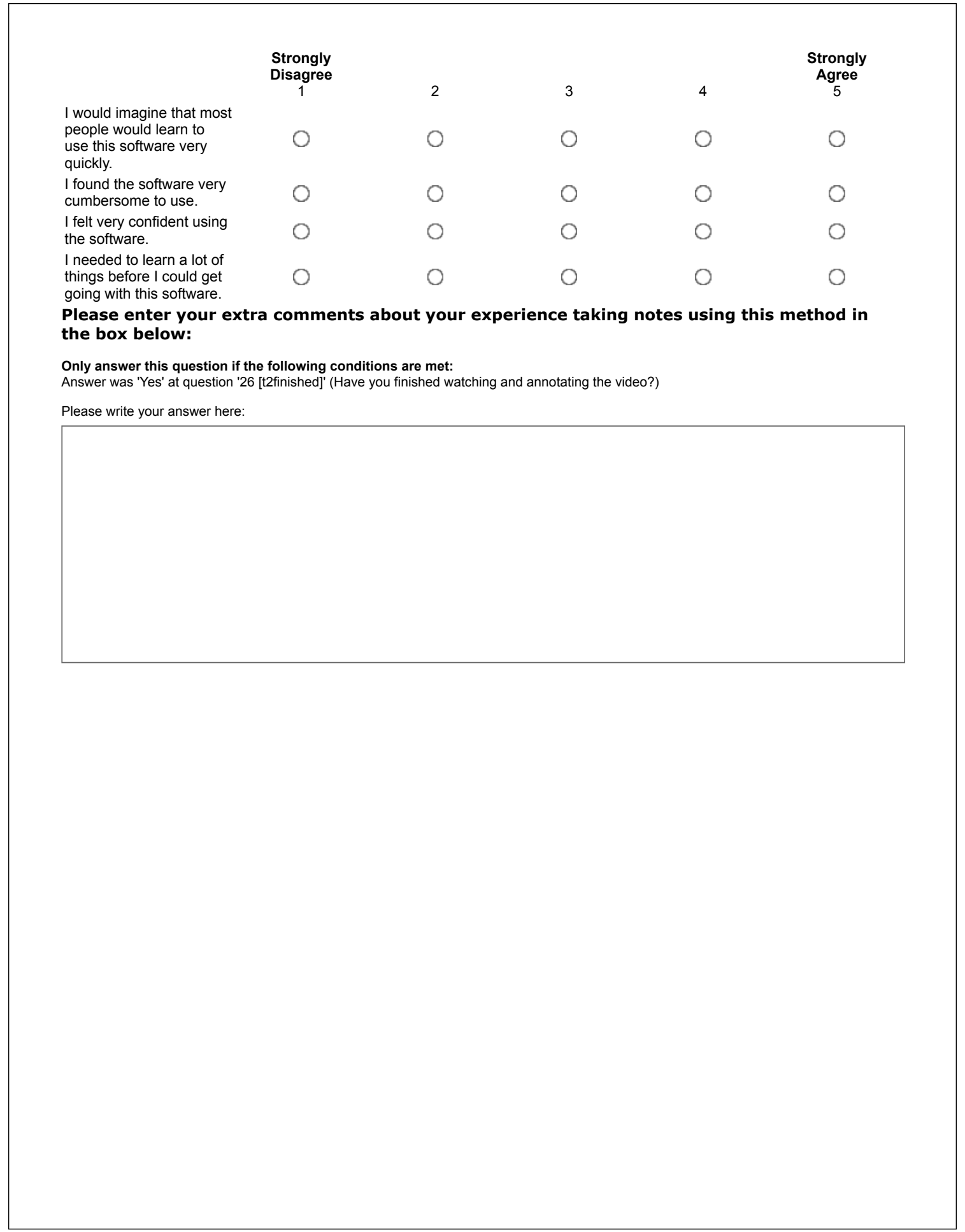


System Usability Scale (SUS) - Post-study Usability Test

\section{NotesKB Demo}

Please check the link below, to give you an introduction of how to use the NotesKB tool to annotate an online educational video:

Please click on the follwoing link to watch the demo: Preview

Have you found the demo useful?

Please choose only one of the following:

Yes

No 


\section{System Usability Scale (SUS) - Post-study Usability Test}

\section{Video \#3: History Of The Tulip Festival}

In this task, we would like you to take notes about an educational video using the NotesKB tool.

\section{Task Instructions}

Please use the following instructions below to carry out the task:

- You have 10 minutes to watch and annotate the video.

- Make sure to include the video's title, website name and author.

- Take notes about (i.e. annotate) the video; and try to type down every proper noun you identify as a hashtag (precede it by the '\#' sign). For example: "This video is about \#Tulip_Festival".

- Moreover, choose the closest annotation type (e.g. C for Concept, ? for Question, Eg for Example, etc) along with their subtype/s whenever appropriate and as you see fit.

\section{Video to Annotate}

Link of educational video: https://youtu.be/kOEtyN-RWAg

If you haven't, please read the instructions of the task.

Please select the number that best describes your experience taking notes using the NotesKB tool:

$*$

Only answer this question if the following conditions are met:

Answer was 'Yes' at question '30 [t1finished]' (Have you finished watching and annotating the video?)

Please choose the appropriate response for each item:

Strongly

Disagree

1

I think that I would like to use this software

frequently.

I found the software

unnecessarily complex.

I thought the software

was easy to use.

I think that I would need

the support of a technical

person to be able to use

this software.

I found the various

functions in this software

were well integrated.

I thought there was too

much inconsistency in

this software.

$\begin{array}{ll}0 & 0 \\ 0 & 0 \\ 0 & 0 \\ 0 & 0 \\ 0 & 0 \\ 0 & 0\end{array}$

3

4

Strongly

$\underset{5}{\text { Agree }}$

0

0

O

O 


\section{System Usability Scale (SUS) - Post-study Usability Test}

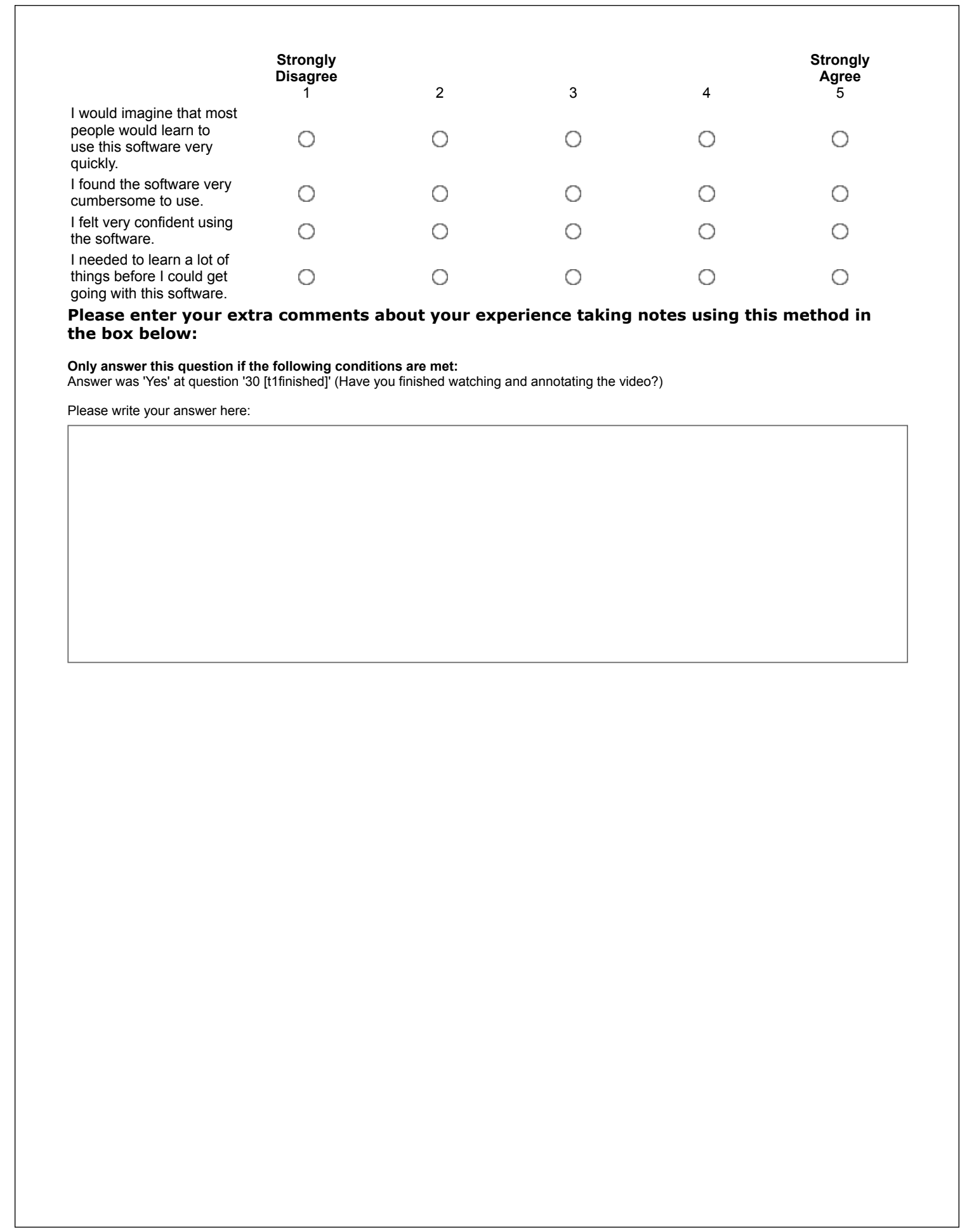


After Scenario Questionnaire (ASQ) - Post-task Usability Test

In this part of the task, please perform the following actions then immediately answer the questions right after you finished performing each one of them. The following steps should help you setup:

1. Saved your current note session.

2. Open the Main View by clicking on the following link: http://localhost:9001/\#/notes.

3. In the Notes tab, make sure there exists a note with the name "What is Programming?".

4. In the Hashtags tab, make sure there exist the following hashtags:

- \#angry_bird

- \#Programming

o \#Khan_Academy

Now, move on to perform the following actions:

Action \# 1: follow the steps below:

- Open note "What is Programming?"

- Move (or re-order) the annotation with the content "This is related to \#Programming" to be on the top of the annotations list.

- Save note.

Please choose the appropriate response for each item:

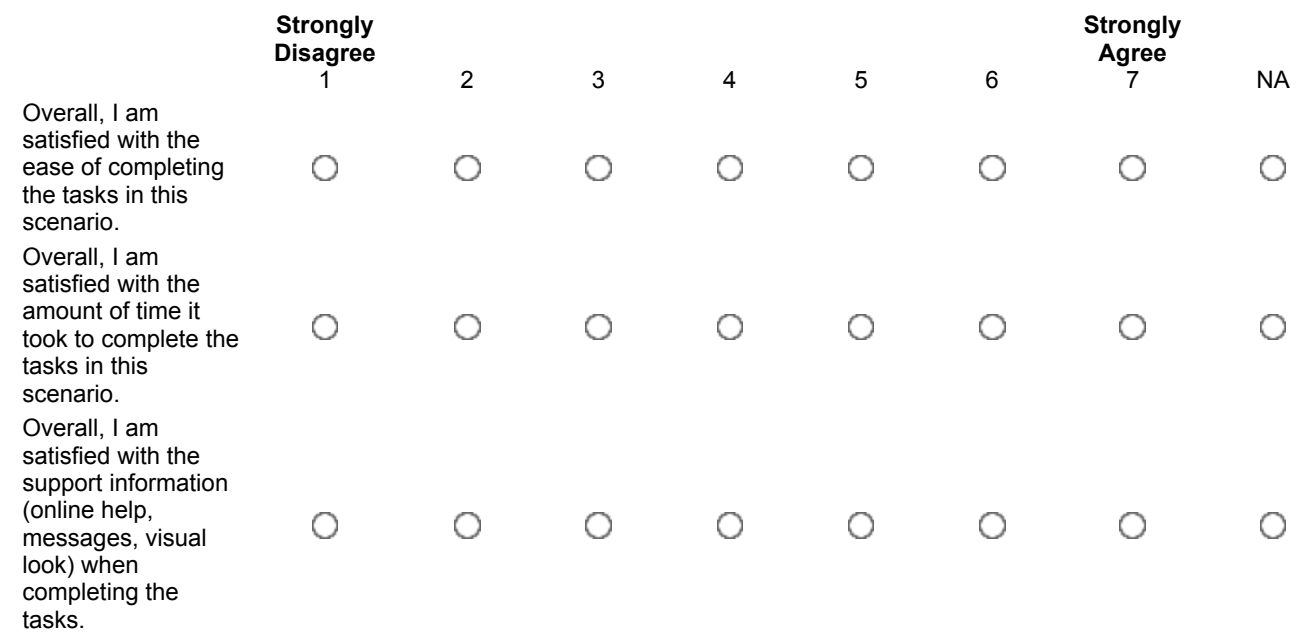

Action \#2: follow the steps below:

- Open note "What is Programming?"

- Remove the annotation with the content "This video is made by \#Khan_Academy."

- Save note.

Please choose the appropriate response for each item:

Strongly

Disagree

2 Strongly Agree 


\section{After Scenario Questionnaire (ASQ) - Post-task Usability Test}

Overall, I am

satisfied with the

ease of completing

the tasks in this

scenario.

Overall, I am

satisfied with the

amount of time it

took to complete the

tasks in this

scenario.

Overall, I am

satisfied with the

support information

(online help,

messages, visual

look) when

completing the

tasks.

Action \#3: follow the steps below:

- Open note "What is Programming?"

- Jump to part of the video that is linked to the hashtag "\#angry_birds".

- Save note.

Please choose the appropriate response for each item:

$\begin{array}{cccccccc}\begin{array}{c}\text { Strongly } \\ \text { Disagree } \\ 1\end{array} & 2 & 3 & 4 & 5 & 6 & \begin{array}{c}\text { Strongly } \\ \text { Agree } \\ 7\end{array} & \text { NA } \\ 0 & 0 & 0 & 0 & 0 & 0 & 0 & 0\end{array}$

satisfied with the

ease of completing

the tasks in this

scenario.

Overall, I am

satisfied with the

amount of time it

took to complete the

tasks in this

scenario.

Overall, I am

satisfied with the

support information

(online help,

messages, visual

look) when

completing the

tasks.

Action \#4: follow the steps below

- Navegate to the Hashtags view.

- Open each of the following hashtags, then find and select the most suitable Wikipedia article related to it:

。 \#khan_academy

○ \#angry_birds

- \#programming 
After Scenario Questionnaire (ASQ) - Post-task Usability Test

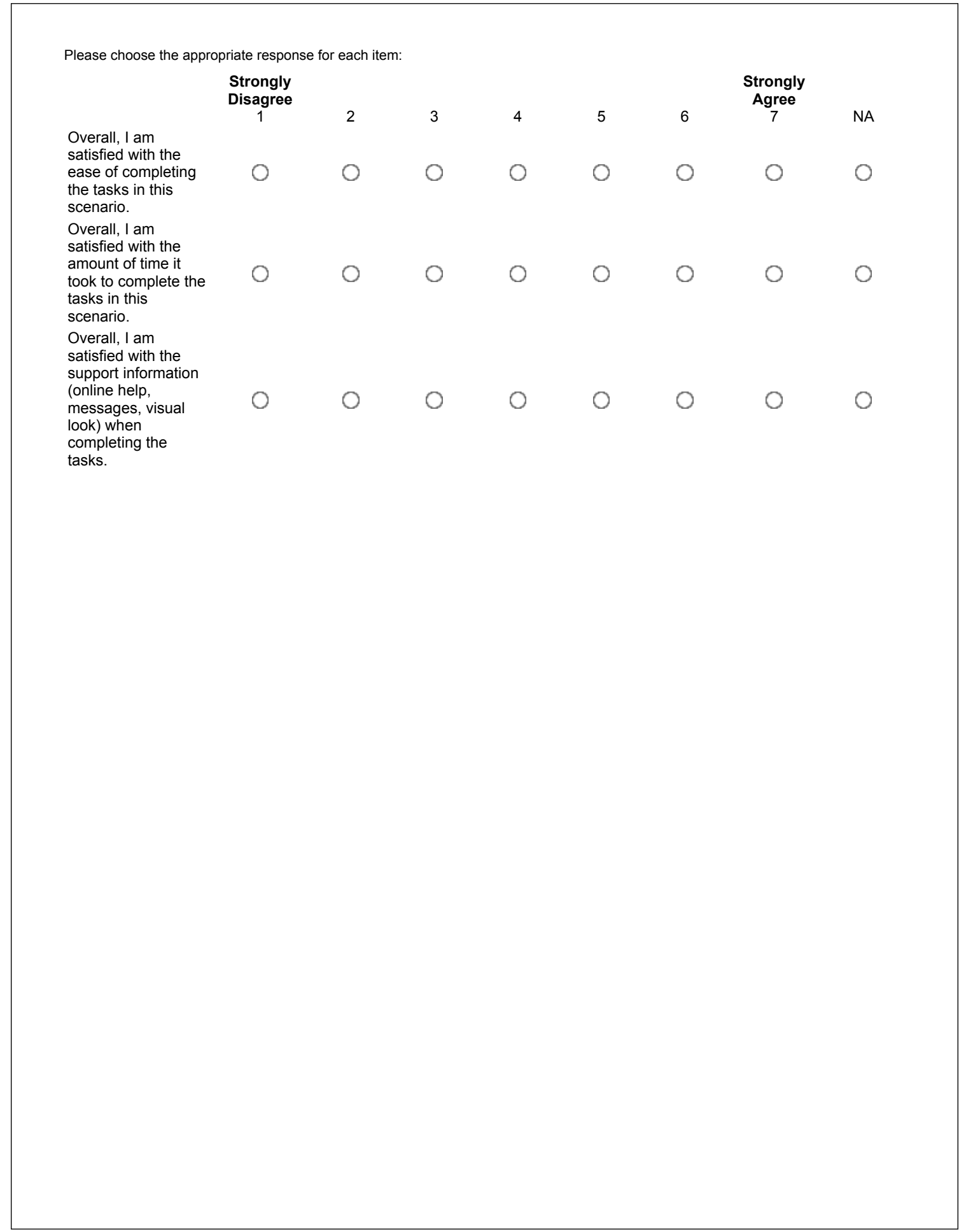




\section{Appendix D}

\section{Video Transcripts}

Each of the following subsections include the video transcript of:

- Video-A - Title: "Introduction to nouns"; Length: 4m 2\%s [78] (Subsection D.1)

- Video-B - Title: "Did You Know? - History Of The Tulip Festival"; Length: $5 m$ 14s [20] (Subsection D.2)

- Video-A - Title: "Firestick farming"; Length: 6m 36s [47] (Subsection D.3)

\section{D.1 Video-A Transcript}

"Hello grammarians. Welcome to the English parts of speech. We're gonna begin with the noun, the lovely wonderful noun, your friend and mine. They're mostly what you're gonna encounter in sentences. Most sentences in English contain at least one noun or a pronoun, but we'll get to pronouns later. A noun is basically anything. And I know that's not an especially helpful definition, but we'll get more specific in a minute. A noun is basically anything at all. Now the way this is taught in traditional grammar is to say that a noun is a person, place or thing, which is fine, I think we can make that a little bit sharper and expand that out by saying that they are, that nouns are people or living things, places, things, or ideas. I think ideas is the one that usually gets left out. So nouns can be people, places, things and ideas. Let's put that in action. So, this is Raul. He is from Argentina. He is a penguin. Raul has big dreams. Now, okay. So, I wanna take these three sentences and find the noun in them using 
the test. So the test is, is it a person or a living thing, a place, a thing, or an idea? And if it's any of those things, then it falls into the category of words in English which we call nouns. So, sentence number one. This is Raul. What is Raul? Well, Raul is a person or a living thing so we're just gonna say person so noun. Next sentence. He is from Argentina. Now Argentina happens to be a place so therefore, it is also a noun. It's a kind of noun called a proper noun, just like Raul is but we'll get to that later. So, Argentina is a noun. Argentina incidentally is a country and the word country is also a noun because it is a thing. So, sentence the third. He is a penguin. Now, a penguin is a living being or a thing so we can say oh yes, penguin, that is a noun as well. So, you've noticed I'm not circling he or this. These words are pronouns, relative pronouns, and they can sometimes behave like nouns, but I wouldn't call them nouns. That'll just get confusing. So, these are pronouns and we'll get to them later. Sentence number four. Raul has big dreams. So, here we have Raul again. We know from the first sentence that it's a person's name. So, we're just gonna say this is also a noun again. And dreams. Now, dreams isn't a person, a place. It's a thing, sure. The reason I put in idea as a fourth category is to get it stuff that you can't pick up. Like for example, so dream, yes, dream is a noun. Dream is maybe a little tangible 'cause it's something you can imagine, but the idea of like a word like bigness. Or if you prefer, you know, size. The size of Raul's plumage was astonishing. Look at that gorgeous plumage. It's a little penguin mohawk. The size of Raul's plumage was astounding. Now in that sentence, size is a noun, but it's not a physical thing, it's not a person, it's not a place, it's not something you can pick up. It's an idea. So, that's why I include this fourth category. So, if you're trying to figure out whether or not a word is a noun, just apply this test. Ask yourself, is it a person, a place, a thing, or an idea? And you, my friend, will be golden. You can learn anything. David out." 


\section{D.2 Video-B Transcript}

"Have you ever wondered why every year the nation's capital is a bloom with millions of colorful tulips? The world's largest tulip festival has an interesting history. One that dates back to World War II. In May of 1940, the Dutch royal family fled to the United Kingdom after the Nazi invasion of the Netherlands. A month later Princess Juliana brought her daughters Beatrix and Irene to Canada for safe harbour. They were housed at Stornoway, now the official residence of the leader of the opposition. It was during this period of exile in Canada at which time Princess Juliana became pregnant. On January 1943, she gave birth to daughter Princess Margaret. The Dutch princess was born at the Ottawa Civic Hospital, and her hospital room was temporarily declared Dutch soil. This ensured the baby's Dutch citizenship. Princess Margaret was the only royal ever to be born in North America. Her birth was a symbol of hope and resistance in the Netherlands. It also sparked celebrations across Canada and helped foster the close relationship between Canada and the Netherlands. As news of her birth spread, the flag of the Netherlands was flown atop Parliament's peace tower and Dutch music rank from the Caroline. Abroad, Canadian troops were also helping the Dutch. In early 1945, the first and second Canadian course carried out an operation to free western and northeastern Netherlands from Nazi occupation. Town by town Canadian troops pushed the German army out of the Netherlands freeing the Dutch and bringing the war closer to its end. It's estimated that over 7,500 Canadian servicemen died while fighting in the Netherlands. After five years of living in exile in Canada, Princess Juliana returned to the Netherlands in the spring of 1945. Upon her return, the princess presented Ottawa with a gift of 100,000 tulip bulbs. This gift was given in recognition of the role of the Canadian troops in the liberation of the Netherlands during World War II and as a show of gratitude for a stay in Canada. Once she became Queen in 1948, Princess Juliana continued to send thousands of tulip bulbs 
Canada every year as an ongoing show of gratitude towards the Canadian people. And year after year the arrival of the tulip bulbs began to spark the curiosity and interest of Canadians across the country. Stunning photographs of these beautiful flowers appeared in newspapers nationwide and as time went on, more and more events began to center around the annual bloom of tulips. In 1953, the first Canadian Tulip Festival was held in Ottawa. It was world-renowned photographer Malak Karsh who suggested to the Ottawa Board of Trade that the festival coincides with the annual bloom. Today the festival's mandate is to preserve and celebrate the special relationship between Canada and Netherlands by displaying the symbolic tulips across the nation's capital. Over the decades the festival has been opened by Governor Generals, prime ministers, and royalty. And the tulip itself has become a symbol of international friendship and celebrates the beauty of spring. It has also become the City of Ottawa official flower. And those 100,000 bulbs have grown into a staggering display of over 2 million flowers. And whose job is it to maintain these impressive tulip displays? The National Capital Commission is the official gardener of the tulip festival and is responsible for designing planting and maintaining the flowers. The colors and varieties of tulips vary. The NCC plans close to 100 different types of tulips in over 40 different locations and in 100 flower beds. And every year 3000 bulbs are given to the Ottawa Civic Hospital where Princess Marguerite was born. Over the years, the Canadian Tulip Festival has grown into the world's largest tulip festival and attracts hundreds of thousands of visitors every year. Canada still receives 20,000 bulbs annually from the Netherlands. And the festival and the tulip itself continue to serve as important symbols of the relationship that exists between the two countries, and now you know." 


\section{D.3 Video-C Transcript}

"Farming, as we now associate the word, has been around for about 7,000 to 10,000 years. And when we think of farming, we imagine a farmer planting seeds, and later harvesting the crops. Or maybe having cattle that they can allow to graze, and then using that cattle for either meat, or milk, or wool. But there's actually a different type of farming that predates this association with I guess what we could call the traditional form of farming. And it predates it by several tens of thousands of years. And we believe that it started with the original inhabitants of Australia. And what they did is - and this is why we call it farming - and because if you think about farming in the most general sense, it's really humans using technology to manipulate their environment so it becomes more suitable for humans. So it becomes more suitable for things that humans might want to eat, or get milk from, or whatever. And this type of farming is called firestick farming. And I think you can already imagine what it might involve. It involves using fire, which is really a form of technology - or it can be a form of technology - using fire to make the environment more suitable for human activity. And so what the original Australians did - the indigenous Australians, or sometimes referred to as the Aboriginal Australians. And if you're wondering where the word Aboriginal comes from, you might recognize some parts of it. Original - you know what that means. The first things. The things that were there from the beginning. And then you have ab, which is Latin for from. So this is literally from

the beginning. So when you say Aboriginal Australians, you're really saying the Australians that were there from the beginning. And so what they would do is, is that we believe if you go back 50,000 or 60,000 years before the first Aboriginal Australians settled Australia, Australia had much more forest. It still has forest. This is a modern picture, obviously, of an Australian forest. But what they did is that they set up controlled burns. And what these controlled burns did is that they cleared away a lot 
of the forest. They cleared away a lot of the brush that's over here, and it made it much more suitable for grassland to develop. And the reason why they liked grassland - so let's make a little cycle here of what they did. So they have controlled burns. Controlled fires. Those controlled fires helped promote grassland. And then once you have grassland, that made the environment more suitable for animals that the original human settlers could essentially live off of. That they could hunt, that they could potentially eat their meat. And so, for example, things like kangaroos. And these supported the human population, which obviously, would then do the controlled burns. And you see here - so we could have started off with something like this. Someone provides a controlled burn. And they were actually pretty scientific about how they did it. They wouldn't just go at the end of summer, when everything was hot, and ready to just blow up, and then start a fire that they couldn't control. They would often do these in seasons knowing that it had a certain level of moisture in the air, it wasn't too hot. And to a large degree, by doing these controlled burns, not only did it provide an environment - kind of do this firestick farming - not only did it provide an environment that was suitable for things like kangaroos, some type of things that humans could eat - but it also prevented major fires. And you still see forest rangers doing this type of thing. And there's some reason to believe that what the original Australians did, on some level, was more nuanced and more fine-tuned than even what we do, in a modern sense, in controlled burns. So these controlled fires also prevented major uncontrollable fires. Because what happens is if you don't have these controlled fires, then you have brush building up, year after year after year. You have stuff building up. And then, when the fires do occur, the uncontrolled fires are less likely to be started during the winter, when the air is cool or when there might be some moisture. They're more likely to occur in the dry season. So you have all this stuff build up. And then when the fire does happen, it happens in the driest season. And then what happens with all of the stuff built up in 
the dry season, it just becomes uncontrollable. One of the byproducts - or actually there are several byproducts of this firestick farming - we believe, is a lot of the grassland in Australia now might have been more forested before. And even when the first European settlers came in the late 1700s, they were kind of surprised when they went into what is now Sydney Harbor and they said, wow, look at all the grassland here. It almost looks like park space. And then they would let their sheep graze there. And they were surprised - because they had driven out the original inhabitants. And then they were surprised when forests just started to grow up in that grassland. And it was because the original Australians were actually controlling that forest growth to make it more inhabitable for things like kangaroos. And then when the English settlers came, they started to have their sheep graze in those grasslands. And it also was responsible for the disappearance, we think, of many major - I guess, for lack of a better word - megafauna. So really large animals that inhabited Australia, for really millions of years, until humans showed up. And this is one of them. It's just neat to look at them. This is called Diprotodon optatum. Or, another way to think of it, the giant wombat. And there's fossils of the giant wombat around 40,000, 50,000 years ago. But they disappeared with humans showing up. And there's multiple ways that you can think about why they disappeared. They might have - and this is probably the case they might have been more dependent on the forest habitat. Or this was a more favourable habitat for them than the grasslands. Maybe because they ate leaves that were high up. Or another thing is, once the forest habitat goes away, they were actually also easier to hunt down. Or either way you think about it, they might have just been hunted by humans. But we do see that with humans coming to the Australian continent, you start to see the disappearance - and this isn't the only one - but there were several major species of megafauna, of super large animals, that disappeared at that time period." 
Appendix E

Examination Supplements 


\section{List of Annotation Types}

\section{Annotation Types}

The follwing table show the types of annotations you may include in your notes along with their shortforms and an example to explain then within context:

\begin{tabular}{|c|c|c|c|}
\hline Type & & Shortform & Example \\
\hline C & Concept & c & $c$ : Artificial Intelligence (AI) \\
\hline Def & Definition & def & $d e f:$ is an intelligence exhibited by machines. \\
\hline$?$ & Question & Q & $Q:$ how far is the sun? \\
\hline Ans & Answer & Ans & Ans: about 149.6 million km. \\
\hline Eg & Example & e.g. & Programming languages (e.g. Java, $\mathrm{C}++$ ) \\
\hline$\neg \mathrm{Eg}$ & Counter Example & !e.g. & !e.g.: HTML isn't a programming language \\
\hline $\mathrm{Ag}$ & Argument & $\arg$ & arg: Spanking is a good child discipline method, because it's old. \\
\hline$\neg \mathrm{Ag}$ & Counter Argument & !arg & !arg: Not all old methods are useful. \\
\hline "Q" & Quote & $" "$ & "I have a dream" - Martin Luther King Jr. \\
\hline
\end{tabular}




\section{Appendix F}

\section{Interview Sample}

The following is a sample of a transcripted interview that was conducted with Participant 12 (P12). From this sample, one can notice the different types of questions asked to the participants the topics of interest. 
Topic \#1: Most/Least

preferred note-taking method

Researcher

Participant-012

Researcher

Participant-012

Researcher

Participant-012

Topic \#2: Opinion on practicing note-taking of online educational videos

Researcher

Participant-012

Topic \#3: Opinion on learning material intrinsic complexity

Researcher

Participant-012

Researcher

Participant-012

Researcher

Participant-012

Researcher

Participant-012

\section{Q. Which of the three methods of you liked the most? And why?}

Ans. I think - I think I preferred OneNote. Because for me personally my notetaking method is very close to plain text and I even found that OneNote had too many features. And I like to have sort of just.. like to not have to use the mouse at all; and just to have kind of.. I just have a box that I can type into and have that be it. I would say - I would say handwriting [PnP], but I'm not very good at that; and it's much faster for me to type.

Q. That's nice. And which of the three methods of you liked the least?

Ahh.. handwriting $[\mathrm{PnP}]$.

Is that because it's not fast enough? Or.

[cont.] It's not fast enough. And also it - it feels slightly futile, because there's no way to index it or access it again without looking at the actual writing that I did. I find if I'm hand writing a note, it's entirely as a memory aid and I'll never refer to it again. Whereas if you're typing a note, well you know at least you have it as a file and you can do stuff with it; like search it.

Q. And.. do you think that taking notes of educational videos is a sound idea or something you would practice on a regular basis?

Ans. No.

Q. Perfect. And which of the videos you think was the longest in terms of time?

Ans. I think the longest was the Tulip one [VideoB].

Q. And lastly, do you think the material presented in videos affected your performance either negatively or positively in anyway?

Ans. Not particularly.. no I don't think so at all actually.

You mean the kind of the fact that they were different, on different subjects?

Yeah.

[cont.] No.

So you think it was kind of neutral?

[cont.] It was neutral. I mean I do think the difficulty was different. But I don't think that - that affected my experience of the act of taking notes. Because I mean none of them, were, you know, particularly difficult material. It wasn't like a lecture on math or something 


\section{Bibliography}

[1] Alain Abran, Adel Khelifi, Witold Suryn, and Ahmed Seffah. 2003. Usability Meanings and Interpretations in ISO Standards. Software Quality Journal 11, 4 (2003), 325-338. https://doi.org/10.1023/A:1025869312943

[2] Samantha Adams Becker, Malcolm Brown, Michele Cummins, and Veronica Diaz. 2017. NMC horizon report: 2017 Higher education edition. (2017). http://cdn.nmc.org/media/2017-nmc-horizon-report-he-EN.pdf

[3] Peter Adamson and Robert Wisnovsky. 2014. Robert Wisnovsky on Commentary Culture. https://www.historyofphilosophy.net/commentary-wisnovsky. (Jul 2014).

[4] Aly AMER. 2006. Reflections on Bloom's Revised Taxonomy Vol 4 (1), pp: 213-230. (2006).

[5] Pierre Andrews, Ilya Zaihrayeu, and Juan Pane. 2012. A classification of semantic annotation systems. Semantic web 3, 3 (2012), 223-248.

[6] Lee C Archie. 2007. The Divisions and Definition of Philosophy. http:// philosophy.lander.edu/intro/what.shtml. (2007). http://philosophy.lander.edu/ intro/what.shtml Accessed April 22, 2015.

[7] David Bargeron, Anoop Gupta, Jonathan Grudin, and Elizabeth Sanocki. 1999. Annotations for streaming video on the Web: system design and usage studies. Computer Networks 31, 11 (1999), 1139 - 1153. https://doi.org/10.1016/ S1389-1286(99)00058-4

[8] BBC News. 2015. Internet used by 3.2 billion people in 2015. http://www.bbc. co.uk/news/technology-32884867. (May 2015). http://www.bbc.co.uk/news/ technology-32884867

[9] J.G. Benjafield, A. Kingstone, and D. Smilek. 2010. Cognition. Oxford University Press. https://books.google.ca/books?id=P6S-ngEACAAJ

[10] Tim Berners-Lee, James Hendler, and Ora Lassila. 2001. The Semantic Web. Scientific American 284, 5 (May 2001), 34-43. http://www.sciam.com/article. cfm?articleID=00048144-10D2-1C70-84A9809EC588EF21

[11] Nigel Bevan. 2009. International standards for usability should be more widely used. Journal of Usability Studies 4, 3 (2009), 106-113. 
[12] Christian Bizer, Tom Heath, and Tim Berners-Lee. 2009. Linked data-the story so far. Semantic Services, Interoperability and Web Applications: Emerging Concepts (2009), 205-227.

[13] Benjamin S Bloom. 1984. The 2 sigma problem: The search for methods of group instruction as effective as one-to-one tutoring. Educational researcher 13, 6 (1984), 4-16.

[14] Marie Bodén. 2015. Contextual considerations for the design of Learner-adaptive computer support in collaborative classrooms. Ph.D. Dissertation. The University of Queensland. https://doi.org/10.14264/uql.2015.1085

[15] Martin Brinkmann. 2016. Google removes Panel support from Chrome. https:// www.ghacks.net/2016/10/19/google-removes-panel-support-from-chrome/. (October 2016).

[16] Manuel Cebrián de la Serna. 2016. Workshop Video annotations. http://gtea. uma.es/congresos/?page_id=1161\&lang=en. (May 2016). http://gtea.uma.es/ congresos/?page_id=1161\&lang=en Accessed June 11, 2017.

[17] Liang-Chu Chen, Jen-Tsung Tseng, Yen-Hsuan Lien, Chia-Jung Hsieh, and I-Chiang Shih. 2016. Exploring a LOD-Based Application for Military Movie Retrieval. Springer International Publishing, Cham, 294-305. https://doi.org/ 10.1007/978-3-319-39396-4_27

[18] Haitao Cheng and Zongmin Ma. 2016. A literature overview of knowledge sharing between Petri nets and ontologies. The Knowledge Engineering Review 31, 3 (July 2016), 239-260. https://doi.org/10.1017/S0269888916000072

[19] Christian Chiarcos, John McCrae, Philipp Cimiano, and Christiane Fellbaum. 2013. Towards Open Data for Linguistics: Linguistic Linked Data. Springer Berlin Heidelberg, Berlin, Heidelberg, 7-25. https://doi.org/10.1007/ 978-3-642-31782-8_2

[20] CPAC. 2014. Did You Know? - History Of The Tulip Festival. (2014). https: //youtu.be/kOEtyN-RWAg

[21] John W. Creswell. 1998. Qualitative inquiry 85 research design : choosing among five approaches. Sage Publications.

[22] Bruna Carolina Rodrigues Cunha, Olibario J. Machado Neto, and Maria da Graca C. Pimentel. 2013. A Heuristic Evaluation of a Mobile Annotation Tool. In Proceedings of the 19th Brazilian Symposium on Multimedia and the Web (WebMedia '13). ACM, New York, NY, USA, 89-92. https://doi.org/10.1145/ 2526188.2526232 
[23] Barney Dalgarno. 2001. Interpretations of constructivism and consequences for Computer Assisted Learning. British Journal of Educational Technology 32, 2 (2001), 183-194. https://doi.org/10.1111/1467-8535.00189

[24] Ton De Jong, Wouter R. Van Joolingen, Adam Giemza, Isabelle Girault, Ulrich Hoppe, Jörg Kindermann, Anders Kluge, Ard W. Lazonder, Vibeke Vold, Armin Weinberger, Stefan Weinbrenner, Astrid Wichmann, Anjo Anjewierden, Marjolaine Bodin, Lars Bollen, Cédric D Ham, Jan Dolonen, Jan Engler, Caspar Geraedts, Henrik Grosskreutz, Tasos Hovardas, Rachel Julien, Judith Lechner, Sten Ludvigsen, Yuri Matteman, Øyvind Meistadt, Bjørge Næss, Muriel Ney, Margus Pedaste, Anthony Perritano, Marieke Rinket, Henrik Von Schlanbusch, Tago Sarapuu, Florian Schulz, Jakob Sikken, Jim Slotta, Jeremy Toussaint, Alex Verkade, Claire Wajeman, Barbara Wasson, Zacharias C. Zacharia, and Martine Van Der Zanden. 2010. Learning by creating and exchanging objects: The SCY experience. British Journal of Educational Technology 41, 6 (2010), 909-921. https://doi.org/10.1111/j.1467-8535.2010.01121.x

[25] Sharon J. Derry, Miriam Gamoran Sherin, and Bruce L. Sherin. 2014. Multimedia Learning with Video (2 ed.). Cambridge University Press, 785-812. https: //doi.org/10.1017/CBO9781139547369.038

[26] Stefan Dietze, Honq Qing Yu, Daniela Giordano, Eleni Kaldoudi, Nikolas Dovrolis, and Davide Taibi. 2012. Linked Education: Interlinking Educational Resources and the Web of Data. In Proceedings of the 27th Annual ACM Symposium on Applied Computing (SAC '12). ACM, New York, NY, USA, 366-371. https://doi.org/10.1145/2245276.2245347

[27] Docebo. 2014. E-Learning Market Trends \& Forecast 2014 - 2016 Report. Online. (March 2014).

[28] Steffi Domagk, Ruth N. Schwartz, and Jan L. Plass. 2010. Interactivity in multimedia learning: An integrated model. Computers in Human Behavior 26, 5 (2010), 1024 - 1033. https://doi.org/10.1016/j.chb.2010.03.003 Advancing Educational Research on Computer-supported Collaborative Learning (CSCL) through the use of gStudy CSCL Tools.

[29] Europeana Labs. 2015. Europeana Connect Annotation Suite. Web page,url=http://labs.europeana.eu/apps/europeanaconnect-annotation. (2015). http://labs.europeana.eu/apps/europeanaconnect-annotation Accessed July 10, 2016.

[30] Chris Evans and Nicola J. Gibbons. 2007. The interactivity effect in multimedia learning. Computers \&G Education 49, 4 (2007), 1147 - 1160. https://doi.org/ 10.1016/j.compedu.2006.01.008 
[31] Michael Färber, Basil Ell, Carsten Menne, and Achim Rettinger. 2015. A Comparative Survey of DBpedia, Freebase, OpenCyc, Wikidata, and YAGO. Semantic Web Journal, July (2015).

[32] Roy T Fielding and Richard N Taylor. 2000. Architectural styles and the design of network-based software architectures. University of California, Irvine Doctoral dissertation.

[33] Keith D. Foote. 2016. A Brief History of Semantics. http://www.dataversity. net/brief-history-semantics/. (2016). Accessed September 28, 2016.

[34] Timothy Gowers, June Barrow-Green, and Imre Leader. 2008. The Princeton Companion to Mathematics. Princeton University Press, Princeton, NJ, USA.

[35] Marco Grassi, Christian Morbidoni, and Michele Nucci. 2012. A Collaborative Video Annotation System Based on Semantic Web Technologies. Cognitive Computation 4, 4 (2012), 497-514. https://doi.org/10.1007/s12559-012-9172-1

[36] Marco Grassi, Christian Morbidoni, Michele Nucci, Simone Fonda, and Francesco Piazza. 2013. Pundit: augmenting web contents with semantics. Literary and linguistic computing 28, 4 (2013), 640-659.

[37] Thomas R. Gruber. 1993. A Translation Approach to Portable Ontology Specifications. Knowl. Acquis. 5, 2 (June 1993), 199-220. https://doi.org/10.1006/ knac.1993.1008

[38] Nicola Guarino and Pierdaniele Giaretta. 1995. Ontologies and knowledge bases: Towards a terminological clarification. In Towards very Large Knowledge bases: Knowledge Building and Knowledge sharing. IOS Press, 25-32.

[39] Ramanathan Guha and Dan Brickley. 2014. RDF Schema 1.1. W3C Recommendation. W3C. http://www.w3.org/TR/2014/REC-rdf-schema-20140225/.

[40] Sherry Hsi and Elliot Soloway. 1998. Learner-centered design: specifically addressing the needs of learners. SIGCHI Bull. 30, 4 (1998), 53-55. https: //doi.org/10.1145/310307.310374

[41] Ali Shariq Imran and Zenun Kastrati. 2016. Pedagogical Document Classification and Organization Using Domain Ontology. Springer International Publishing, Cham, 499-509. https://doi.org/10.1007/978-3-319-39483-1_45

[42] Jeff Johnson, Teresa L. Roberts, William Verplank, David Canfield Smith, Charles H. Irby, Marian Beard, and Kevin Mackey. 1989. The xerox star: A retrospective. Computer 22, 9 (1989), 11-26. 
[43] Yasuhiro Kakinuma and Akihiro Kashihara. 2016. A Micro-Web Involving Learning Scenario Generation with Linked Open Data for Web-Based Investigative Learning. Springer International Publishing, Cham, 344-355. https://doi.org/10.1007/978-3-319-39483-1_32

[44] Slava Kalyuga. 2007. Expertise Reversal Effect and Its Implications for LearnerTailored Instruction. Educational Psychology Review 19, 4 (2007), 509-539. https://doi.org/10.1007/s10648-007-9054-3

[45] Maedeh Assadat Kazemitabar, Stephen Bodnar, Peter Hogaboam, Yuxin Chen, Juan Pablo Sarmiento, Susanne P. Lajoie, Cindy Hmelo-Silver, Ricki Goldman, Jeffrey Wiseman, and Lapki Chan. 2016. Creating Instructor Dashboards to Foster Collaborative Learning in On-Line Medical Problem-Based Learning Situations. Springer International Publishing, Cham, 36-47. https://doi.org/10. 1007/978-3-319-39483-1_4

[46] Mumtaz Khan, Shah Khusro, and Irfan Ullah. 2016. On Annotation of Video Content for Multimedia Retrieval and Sharing. International Journal of Computer Science and Information Security 14, 3 (2016), 198.

[47] Salman Khan. 2011. Firestick farming. (2011). https://youtu.be/mwUyaeWxJhA

[48] Soroush Khanlou. 2014. Model View Whatever. http://khanlou.com/2014/03/ model-view-whatever/. (March 2014).

[49] Atanas Kiryakov, Borislav Popov, Ivan Terziev, Dimitar Manov, and Damyan Ognyanoff. 2004. Semantic annotation, indexing, and retrieval. Web Semantics: Science, Services and Agents on the World Wide Web 2, 1 (2004), 49 - 79. https://doi.org/10.1016/j.websem.2004.07.005

[50] Scott R. Klemmer, Björn Hartmann, and Leila Takayama. 2006. How Bodies Matter: Five Themes for Interaction Design. In Proceedings of the 6th Conference on Designing Interactive Systems (DIS '06). ACM, New York, NY, USA, 140-149. https://doi.org/10.1145/1142405.1142429

[51] Markus Lanthaler, David Wood, and Richard Cyganiak. 2014. RDF 1.1 Concepts and Abstract Syntax. W3C Recommendation. W3C. http://www.w3.org/TR/2014/REC-rdf11-concepts-20140225/.

[52] L. Leung. 2008. Digital Experience Design: Ideas, Industries, Interaction. Intellect. https://books.google.co.in/books?id=Yi2NPwAACAAJ

[53] James R. Lewis. 2012. Usability Testing. John Wiley \& Sons, Inc., 1267-1312. https://doi.org/10.1002/9781118131350.ch46 
[54] James R. Lewis and Jeff Sauro. 2009. The Factor Structure of the System Usability Scale. Springer Berlin Heidelberg, Berlin, Heidelberg, 94-103. https: //doi.org/10.1007/978-3-642-02806-9_12

[55] Yunjia Li, Mike Wald, Tope Omitola, Nigel Shadbolt, and Gary Wills. 2012. Synote: weaving media fragments and linked data. (March 2012). https:// eprints.soton.ac.uk/335328/

[56] Lijia Lin and Robert K. Atkinson. 2011. Using animations and visual cueing to support learning of scientific concepts and processes. Computers 85 Education 56, 3 (2011), 650 - 658. https://doi.org/10.1016/j.compedu.2010.10.007

[57] Zongmin Ma. 2006. Web-Based Intelligent E-Learning Systems: Technologies and Applications. Information Science Publishing.

[58] Jason Mander. 2016. Daily time spent on social networks rises to 1.72 hours. (2016). http://www.globalwebindex.net/blog/ daily-time-spent-on-social-networks-rises-to-1-72-hours

[59] Sergio Martin, Gabriel Diaz, Elio Sancristobal, Rosario Gil, Manuel Castro, and Juan Peire. 2011. New technology trends in education: Seven years of forecasts and convergence. Computers \& Education 57, 3 (2011), 1893 - 1906. https://doi.org/10.1016/j.compedu.2011.04.003

[60] Thomas Mautner. 1998. The Penguin Dictionary of Philosophy (Penguin Dictionary). Penguin Books.

[61] Pablo N. Mendes. 2013. Adaptive Semantic Annotation of Entity and Concept Mentions in Text. Ph.D. Dissertation. Wright State University. http: //corescholar.libraries.wright.edu/knoesis/1033

[62] Negin Mirriahi, Daniyal Liaqat, Shane Dawson, and Dragan Gašević. 2016. Uncovering student learning profiles with a video annotation tool: reflective learning with and without instructional norms. Educational Technology Research and Development 64, 6 (01 Dec 2016), 1083-1106. https://doi.org/10.1007/ s11423-016-9449-2

[63] Roberto Navigli and Paola Velardi. 2004. Learning Domain Ontologies from Document Warehouses and Dedicated Web Sites. Computational Linguistics 30, 2 (June 2004), 151-179. https://doi.org/10.1162/089120104323093276

[64] C. Nithya and K. Saravanan. 2014. Semantic Annotation and Search for Educational Resources Supporting Distance Learning. CoRR abs/1403.0068 (2014). http://arxiv.org/abs/1403.0068

[65] Donald A Norman. 2013. The design of everyday things: Revised and expanded edition. Basic books. 
[66] Cathie Norris and Elliot Soloway. 2016. Learner-Centered Design: Powering the Coming Golden Age of Educational Software. THE Journal (2016).

[67] Ofcom. 2015. Time spent online doubles in a decade. (2015). http://media. ofcom.org.uk/news/2015/time-spent-online-doubles-in-a-decade/

[68] Lukasz Ostrowski, Markus Helfert, and Nelson Gama. 2014. Ontology engineering step in design science research methodology: a technique to gather and reuse knowledge. Behaviour $\&$ Information Technology 33, 5 (2014), 443-451. https://doi.org/10.1080/0144929X.2013.815276 arXiv:http://dx.doi.org/10.1080/0144929X.2013.815276

[69] Sharon Oviatt, Alex Arthur, and Julia Cohen. 2006. Quiet Interfaces That Help Students Think. In Proceedings of the 19th Annual ACM Symposium on User Interface Software and Technology (UIST '06). ACM, New York, NY, USA, 191-200. https://doi.org/10.1145/1166253.1166284

[70] Fred Paas and John Sweller. 2014. Implications of Cognitive Load Theory for Multimedia Learning (2 ed.). Cambridge University Press, 27-42. https: //doi.org/10.1017/CBO9781139547369.004

[71] Jeffrey M. Perkel. 2015. Annotating the scholarly web. Nature 528, 7580 (1 Dec. 2015), 153-154. https://doi.org/10.1038/528153a

[72] Dorian Peters. 2014. Interface design for learning : design strategies for learning experiences. New Riders.

[73] Teodora Petkova. 2015. Content Writing in the Semantic Web. (2015). Accessed November 15, 2015.

[74] Duncan Pritchard. 2013. What is this thing called knowledge? (3 ed.). Routledge.

[75] Chris Quintana, Joseph Krajcik, and Elliot Soloway. 2000. Exploring a Structured Definition for Learner-Centered Design. In Fourth International Conference of the Learning Sciences. 256-263.

[76] C. Quintana, J. Krajcik, and E. Soloway. 2001. Issues and methods for evaluating learner-centered scaffolding. In Proceedings IEEE International Conference on Advanced Learning Technologies. 353-356. https://doi.org/10.1109/ICALT.2001. 943943

[77] Stephan Rauh. 2015. Model-View-Whatever. https://www.beyondjava.net/blog/ model-view-whatever/. (October 2015).

[78] David Rheinstrom. 2016. Introduction to nouns. (2016). https://youtu.be/ UejW-WQpujs 
[79] Peter J. Rich and Michael Hannafin. 2009. Video Annotation Tools. Journal of Teacher Education 60, 1 (2009), 52-67. https://doi.org/10.1177/ 0022487108328486 arXiv:http://dx.doi.org/10.1177/0022487108328486

[80] Mary Beth Rosson and John M. Carroll. 2002. Usability Engineering: Scenariobased Development of Human-computer Interaction. Morgan Kaufmann Publishers Inc., San Francisco, CA, USA.

[81] Priti Srinivas Sajja and Rajendra Akerkar. 2012. Mining sentiment using conversation ontology. Advancing information management through semantic web concepts and ontologies (2012), 302-315.

[82] Jeff Sauro. 2011. A Practical Guide to the System Usability Scale: Background, Benchmarks \& Best Practices. CreateSpace Independent Publishing Platform.

[83] Jeff Sauro and James R. Lewis. 2016. Quantifying the User Experience (Second Edition) (second edition ed.). Morgan Kaufmann, Cambridge, MA. https: //doi.org/10.1016/B978-0-12-802308-2.00013-8

[84] Daniel L Schwartz and Kevin Hartman. 2007. It is not television anymore: Designing digital video for learning and assessment. Video research in the learning sciences (2007), 335-348.

[85] Nigel Shadbolt, Wendy Hall, and Tim Berners-Lee. 2006. The Semantic Web Revisited. IEEE Intelligent Systems 21, 3 (2006), 96-101.

[86] Elena Simperl. 2012. Creating and using ontologies. http://videolectures.net/ eswc2012_simperl_ontologies/. (2012). http://videolectures.net/eswc2012_ simperl_ontologies/ Tutorial presented at the meeting of 2nd ESWC Summer School, Heraklion 2012.

[87] Elena Simperl. 2015. Building and using ontologies. http://videolectures. net/eswc2015_simperl_building_ontologies/. (2015). http://videolectures.net/ eswc2015_simperl_building_ontologies/ Tutorial presented at the meeting of the 5th ESWC Summer School, Crete 2015.

[88] Steffen Staab, Alexander Maedche, and Siegfried Handschuh. 2001. An Annotation Framework for the Semantic Web. In Proc. of The First International Workshop on MultiMedia Annotation, Tokyo, Japan, S. Ishizaki (Ed.).

[89] Angus Stevenson. 2010. Oxford Dictionary of English (3 ed.). Oxford University Press. https://doi.org/10.1093/acref/9780199571123.001.0001

[90] Rudi Studer, V. Richard Benjamins, and Dieter Fensel. 1998. Knowledge Engineering: Principles and Methods. Data Knowl. Eng. 25, 1-2 (March 1998), 161-197. https://doi.org/10.1016/S0169-023X(97)00056-6 
[91] Bimba Andrew Thomas, Norisma Idris, Ahmed Al-Hnaiyyan, Rohana Binti Mahmud, Ahmed Abdelaziz, Suleman Khan, and Victor Chang. 2016. Towards knowledge modeling and manipulation technologies: a survey. (December 2016), 857-871 pages. https://eprints.soton.ac.uk/396010/

[92] Jehan Tremback. 2013. Embedded Annotations. https://hypothes. is/blog/embedded-annotations/. (2013). https://hypothes.is/blog/ embedded-annotations/ Accessed September 20, 2017.

[93] David A. Truncellito. 2007. Epistemology. http://www.iep.utm.edu/epistemo. (2007). http://www.iep.utm.edu/epistemo Accessed March 12, 2017.

[94] Mike Uschold. 1998. Knowledge Level Modelling: Concepts and Terminology. The Knowledge Engineering Review 13, 1 (March 1998), 5-29. https://doi.org/ $10.1017 / \mathrm{S} 0269888998001040$

[95] W3C. 2016. Linked Data. http://www.w3.org/standards/semanticweb/data. (2016).

[96] W3C. 2016. Semantic Web. https://www.w3.org/standards/semanticweb/. (2016). Accessed September 15, 2016.

[97] Mike Wald. 2010. Synote: accessible and assistive technology enhancing learning for all students. Computers Helping People with Special Needs (2010), 177-184.

[98] Michelle Q. Wang Baldonado, Allison Woodruff, and Allan Kuchinsky. 2000. Guidelines for Using Multiple Views in Information Visualization. In Proceedings of the Working Conference on Advanced Visual Interfaces (AVI '00). ACM, New York, NY, USA, 110-119. https://doi.org/10.1145/345513.345271

[99] Colin Ware. 2012. Information Visualization: Perception for Design (3 ed.). Morgan Kaufmann Publishers Inc., San Francisco, CA, USA.

[100] M. J. Weal, D. T. Michaelides, K. Page, D. C. De Roure, E. Monger, and M. Gobbi. 2012. Semantic Annotation of Ubiquitous Learning Environments. IEEE Transactions on Learning Technologies 5, 2 (April 2012), 143-156. https: //doi.org/10.1109/TLT.2011.37

[101] Dan Whaley. 2013. Annotating Knowledge. https://www.youtube.com/watch? $\mathrm{v}=$ ChXRuuePrDQ. (February 2013). https://www.youtube.com/watch? $\mathrm{v}=$ ChXRuuePrDQ Accessed September 27, 2016.

[102] Beverly Park Woolf. 2009. Building Intelligent Interactive Tutors Studentcentered Strategies for Revolutionizing E-learning. Morgan Kaufmann. 
[103] Hong Qing Yu, Carlos Pedrinaci, Stefan Dietze, and John Domingue. 2012. Using Linked Data to Annotate and Search Educational Video Resources for Supporting Distance Learning. IEEE Trans. Learn. Technol. 5, 2 (Jan. 2012), 130-142. https://doi.org/10.1109/TLT.2012.1

[104] Liyang Yu. 2014. A Developer's Guide to the Semantic Web, Second Edition. Springer. https://doi.org/10.1007/978-3-662-43796-4

[105] Xiangmin Zhang. 2016. Searching Interactions and Perceived Learning. Springer International Publishing, Cham, 245-255. https://doi.org/10.1007/ 978-3-319-39483-1_23 